\title{
Disulfide Bridging the Gap between Src and Cortactin: A New Paradigm in SH2 Domain-mediated Signaling
}

\author{
Jason VanBuren Evans \\ West Virginia University
}

Follow this and additional works at: https://researchrepository.wvu.edu/etd

\section{Recommended Citation}

Evans, Jason VanBuren, "Disulfide Bridging the Gap between Src and Cortactin: A New Paradigm in SH2 Domain-mediated Signaling" (2013). Graduate Theses, Dissertations, and Problem Reports. 427.

https://researchrepository.wvu.edu/etd/427

This Dissertation is protected by copyright and/or related rights. It has been brought to you by the The Research Repository @ WVU with permission from the rights-holder(s). You are free to use this Dissertation in any way that is permitted by the copyright and related rights legislation that applies to your use. For other uses you must obtain permission from the rights-holder(s) directly, unless additional rights are indicated by a Creative Commons license in the record and/ or on the work itself. This Dissertation has been accepted for inclusion in WVU Graduate Theses, Dissertations, and Problem Reports collection by an authorized administrator of The Research Repository @ WVU.

For more information, please contact researchrepository@mail.wvu.edu. 


\title{
Disulfide Bridging the Gap between Src and Cortactin: A New Paradigm in SH2 Domain-mediated Signaling
}

\section{Jason VanBuren Evans}

\section{Dissertation Submitted to the School of Medicine at West Virginia University In Partial Fulfillment of the Requirements for the Degree of \\ Doctor of Philosophy in Cancer Cell Biology}

\author{
Laura Gibson, Ph.D., Chair \\ Jun Liu, Ph.D. \\ Karen Martin, Ph.D. \\ Yong Qian, Ph.D. \\ Robert Wysolmerski, Ph.D. \\ Scott Weed, Ph.D., Mentor \\ Cancer Cell Biology Program \\ Morgantown, West Virginia \\ 2013
}

Keywords: Src, SH2, Cortactin, Disulfide 


\begin{abstract}
Disulfide Bridging the Gap between Src and Cortactin: A New Paradigm in $\mathrm{SH} 2$ Domain-mediated Signaling
\end{abstract}

Jason V. Evans

Src and cortactin are cytoplasmic proteins that are implicated in cancer progression and metastasis. Src is a non-receptor tyrosine kinase that also regulates normal cell homeostasis through phosphorylation of multiple downstream substrates. Cortactin is an actin binding protein and nucleation promoting factor that promotes the formation of stable branching networks within the actin cytoskeleton. Together, these proteins work in concert to promote the invasive and metastatic potential of tumor cells due to tyrosine phosphorylation of cortactin by Src. However, the mechanistic details of the interaction between Src and cortactin have never been elucidated. Collectively, this work aims to define how Src and cortactin interact to drive tumor cell invasion. The first study examines the mechanism of interaction between Src and cortactin, whereby the Src $\mathrm{SH} 2$ domain interacts with cortactin via a disulfide bond formation and that this binding event is necessary for the formation of pro-invasive invadopodia. The work here also defines a paradigm shift in how $\mathrm{SH} 2$ domain-containing proteins interact with reciprocal binding partners. The second study characterizes potential structural changes in cortactin resultant from phosphorylation by extracellular regulated protein kinases (ERKs) in order to gain structural insight into how cortactin conformation is regulated downstream of growth factor-mediated signaling events. The final study focuses on the creation of cortactin biosensor to directly monitor changes in cortactin conformation using FRET-based imaging. Taken together, these studies provide novel insights into the molecular events involved in regulating invasive tumor cell signal transduction and overall control of cortical actin dynamics during tumor metastasis. 


\section{Dedication}

I would like to dedicate this to my wife, Tracie, and daughter, Roselyn. The two of you have been an infinite source of inspiration, courage, and strength. My accomplishments exist because of your love and support. 


\section{Acknowledgements}

I would first like to acknowledge my wonderful wife, Tracie, for her continuous encouragement and support throughout this stage of my career. Beyond that, we have shared many memories with one another throughout our years together at WVU and in Morgantown. May this only be the beginning of our adventure in life! Together we have conquered many obstacles and have brought into this world a beautiful little girl, Roselyn Kate. This blessing to us has given me a new meaning and perspective on life. For this, I am grateful to you both for the person you have shown me I can be.

I would also like to also thank my parents, Johnny and Linda Evans, for their 30+ years of love and support of myself and my dreams. Their teachings on hard work and being driven have structured the core of my personality that has allowed me to succeed in academic life as well as life in general. For that, you have my eternal thanks.

Next I would like to thank my mentor, Scott Weed, Ph.D. Thank you ever so much for the opportunity to pursue my dreams in your lab. I have learned much from you in these past seven years, in both matters of science and research, as well as life. Thank you for your encouragement and confidence in my abilities. It will not go forgotten.

To my committee, I would like to thank Dr. Laura Gibson, Dr. Jun Liu, Dr. Karen Martin, Dr. Yong Qian, and Dr. Robert Wysolmerski for sharing their experience and insight over the years. All of you have truly made committee meetings a source of knowledge and exciting ideas. Thank you.

To past and present members of the Weed Lab, thank you for the shared laughter and pain the years have brought our way. It would have been a tough road to travel alone.

Lastly, I would like to thank the Cancer Cell Biology program, West Virginia University, and the great state of West Virginia. To the CCB program, thank you for the opportunities over the years and providing the vessel to hone my scientific skills. In my experiences, our impact as a program and Cancer Center reaches well beyond our halls. To WVU, the experiences I've had since I started my undergraduate career in 2000 have been fantastic! The friends made and good times shared are memories that I will undoubtedly hold close to my heart for the rest of my life. Last, but most certainly not least, to the state of West Virginia. The state I was born and raised. The state I played in the mountains and streams as a child. The state that molded me into the person I am today. To you, I am forever grateful for your rugged beauty and I pray that your country roads always lead me home. 


\section{Table of Contents}

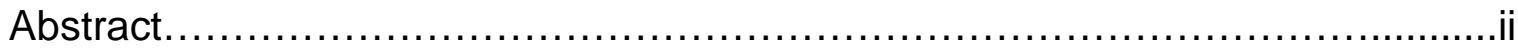

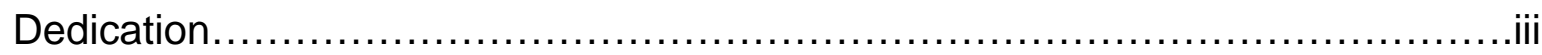

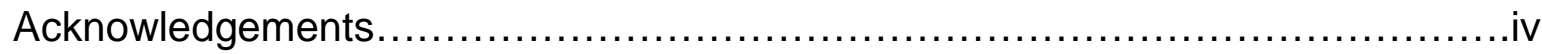

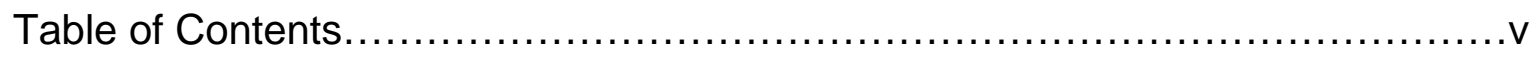

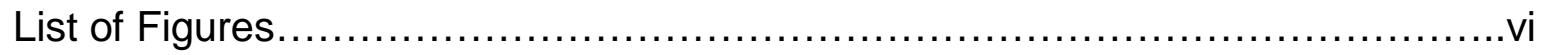

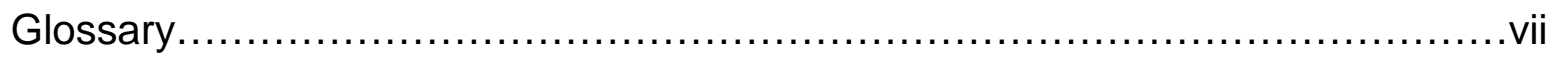

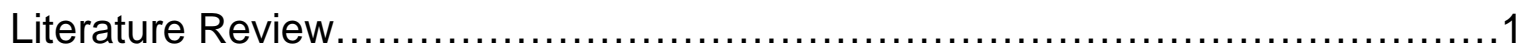

Src: Function and Domain-based Regulation

Cortactin: Integrator of Actin Cytoskeletal Signaling

Src and Cortactin: Phosphoregulation of the Actin Cytoskeleton

SH2 Domains: Non-Conventional Interactions

Study 1: Src Binds Cortactin Through a SH2 Domain...................................51 Cystine-mediated Linkage

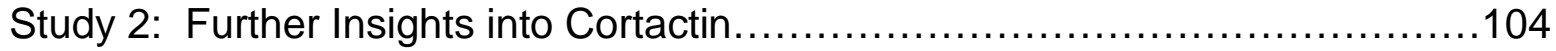
Conformational Regulation

Study 3: Construction of a FRET-based Cortactin Biosensor...................115

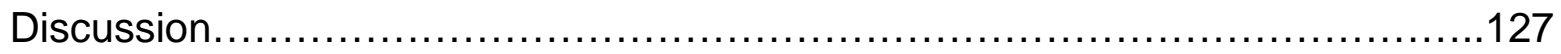

Appendix

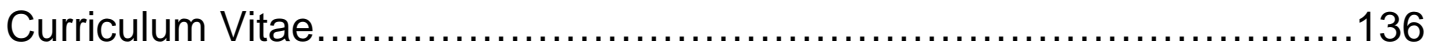




\section{List of Figures}

\section{Literature Review}

1. Src kinase structure and role in tumor invasion.

2. Cortactin structure and role in tumor invasion.

3. Src and cortatin work in concert to regulate cell motility and invasion.

\section{Study 1}

1. Src SH2 Binding to Cortactin Does not Involve Tyrosine Phosphorylation and Binds Cortactin Repeats 1 and 5

2. Cortactin Cysteines 112 and 246 are Required for Src SH2 Domain Binding

3. Cysteine-containing Cortactin Peptides Dock Within the $\mathrm{Src} \mathrm{SH} 2$ Phosphotyrosine Binding Region

4. Binding and Phosphorylation of Cortactin by Src is Redox Dependent and Requires Src C185

5. Src C-185 Forms a Cystine Bond with Cortactin C112 and C246

6. Cortactin C112/246 is Essential for Phosphotyrosine-based Cortactin Function

7. Model of cysteine-mediated interactions in cortactin regulation

Table 1. Observed and predicted ion masses of GST-Src-SH2, GST-Src-SH2 + cortactin C112 and GST-Src-SH2 + cortactin C246 tryptic peptides

\section{Supplemental Figures:}

1. Src Binding to Cortactin Requires the $\mathrm{SH} 2$ Domain and is Phosphotyrosine Independent

2. Phosphorylation of Recombinant Cortactin by Src and Mapping of $\mathrm{Src} \mathrm{SH} 2$ Binding to the Cortactin Amino Terminal Domain

3. Schematic Diagram of Cortactin Constructs Used in this Study

4. Src SH2 Domain Does not Bind to the $5^{\text {th }}$ Cortactin Repeat 
5. Src SH2 R175A Retains Binding to Cortactin

6. Y-ion Analysis of Cystine bonding between Src C185 and cortactin C112 and C246

7. Expression of Cortactin Mutants in Cortactin Knockdown Cells and F-actin Co-sedimentation of Cortactin Mutants

\section{Study 2}

1. Analysis of cortactin secondary structure following ERK1 phosphorylation

\section{Study 3}

1. Design and testing of a FRET-based cortactin Biosensor

2. Creation and schematic of function of cortactin biosensor and controls 


\section{Glossary}

\begin{tabular}{ll} 
3D & Three Dimensional \\
$\AA$ & Angstrom \\
A & Alanine \\
ADF & Actin Depolymerizing Factor \\
AFAP & Actin Filament Associated Protein \\
Arp & Actin related protein \\
ATP & Adenosine Triphosphate \\
BCR & Breakpoint Cluster Region \\
C & Celsius/Cysteine \\
C- & Cellular \\
CAS & Crk-associated substrate \\
CBL & Casitas B-lineage \\
CD & Circular Dichroism \\
CDK & Cyclin Dependent Kinase \\
CFP & Cyan Fluorescent Protein \\
CSK & C-Src Kinase \\
CT & Carboxy-Terminal \\
D & Aspartic Acid \\
Da & Dalton \\
DCM & Double Cysteine Mutant \\
DLC-1 & Deleted in Lung Caner-1 \\
DTT & Dithiothreitol \\
E & Glutamic Acid \\
EAT2 & EWS-FLI1-activated transcript 2 \\
ECM & Extracellular Matrix \\
EGF & Epidermal Growth Factor \\
EGFR & Epidermal Growth Factor Receptor \\
Erk & Extracellular Signal-regulated Protein Kinase \\
F & Phenylalanine \\
FAK & Focal Adhesion Kinase \\
FGF & Fibroblast Growth Factor \\
FRET & Fluorescent Resonance Energy Transfer \\
GAP & GTPase Activating Protein \\
GDP & Guanidine Diphosphate \\
GEF & Guanidine Exchange Factor \\
Grb & Growth factor receptor binding \\
GST & Glutathione-S-Transferase \\
GTP & Guanidine Triphosphate \\
HDAC & Histone Deacetylase \\
HNSCC & Head and Neck Squamous Cell Carcinoma \\
Itk & IL2-inducible T-cell kinase \\
JNK & Jun N-terminal Kinase \\
K & Lysine \\
& \\
\hline
\end{tabular}




\begin{tabular}{ll} 
M & Methionine \\
MAPK & Mitogen-activated Protein Kinase \\
MEK & MAPK/Erk Kinase \\
MMP & Matrix Metalloproteinase \\
N & Asparagine \\
NADPH & Nicotinamide Adenine Dinucleotide Phosphate \\
Nox & NADPH Oxidases \\
NPF & Nucleation Promoting Factor \\
NT & Aminio-terminus \\
NTA & N-terminal Acidic \\
N-WASP & Neuronal Wiskott-Aldrich Syndrome Protein \\
P & Proline \\
Pal & Peptidoglycan-associated Lipoprotein \\
PAK & p21-activated Kinases \\
PCAF & P300/CBP-associated factor \\
PDGFR & Platelet-derived Growth Factor Receptor \\
PLC & Protein Phospholipase \\
PTP & Protein Tyrosine Phosphatase \\
pY & Phospho-tyrosine \\
R & Arginine \\
ROS & Reactive Oxygen Species \\
S & Serine \\
SAP & SLAM-associated Protein \\
SDS-PAGE & Sodium Dodecyl Sulfate-Polyacrylamide Gel Electrophoresis \\
SH1 & Src Homology 1 \\
SH2 & Src Homology 2 \\
SH3 & Src Homology 3 \\
SH4 & Src Homology 4 \\
SLAM & Signaling Lymphocyte-Activation Molecule \\
SOCS1 & Suppressor of Cytokine Signaling 1 \\
STAT & Signal Transducer and Activator of Transcription \\
T & Threonine \\
TYM & Triple Tyrosine Mutant \\
V & Valine \\
V- & Viral \\
W & Tryptophan \\
WIP & WASP Interactin Protein \\
WT & Wild-type \\
X & Any amino acid \\
Y & Tyrosine \\
YFP & Yellow Fluorescent Protein \\
& \\
\hline
\end{tabular}




\section{Literature Review}

\section{Src: Function and Domain-based Regulation}

In the early 1900s, Peyton Rous discovered a virus that caused the formation and growth of tumors in chickens [1]. Named Rous sarcoma virus, it was later discovered that the transmissible virus encoded a gene product, v-src, which was responsible for the cellular transformation observed in infected cells. A cellular homologue, known as c-Src (Src from this point on), is found in normal tissues and has been vastly studied over decades, where it has been shown to play a critical role in normal and human neoplastic cell function [2].

Src does not share the transforming ability of $\mathrm{v}$-Src. This is due to structural differences that allow Src to be precisely regulated. Src is a non-receptor tyrosine kinase that acts on other proteins by phosphorylating tyrosine residues on target ligands to induce a litany of downstream effects. Molecular cloning and sequence analysis initially revealed important structural clues regarding Src regulation. Beginning at the amino (N-) terminus, Src contains a myristoylation site that tethers the kinase to the inner leaflet of the plasma membrane and is key for proper subcellular localization and function. Carboxyl (C-) terminal to the myrostolyation site is a Src homology (SH)3 domain. This domain functions to facilitate protein-protein interactions by recognizing and binding to canonical P-X-X-P peptide motifs. This allows for the specific recognition and subsequent phosphorylation of Src substrates. The SH3 domain is also important in governing Src conformation and activity (see below). Following the SH3 domain is second protein-protein binding motif termed the Src homology 2 (SH2) domain. Traditionally the $\mathrm{SH} 2$ domain mediates protein interactions by binding to phosphorylated 
tyrosine residues through a hydrophobic binding pocket that contains a key, conserved, positively charged arginine that facilitates the interaction with the negatively charged oxygen moieties of the phospho-group on the tyrosine residues. Adjacent to the Src $\mathrm{SH} 2$ domain is the Src homology 1 (SH1), more commonly referred to as the kinase, domain. This is the catalytic region of the kinase, consisting of an N-terminal and Cterminal lobe connected by an activation loop that is autophosphorylated at the key tyrosine (Y) 418 in the human enzyme (Y416 in the avian form). Autophosphorylation of Y418 is essential for Src to attain full catalytic function towards exogenous substrates. Lastly, Src possesses a C-terminal negative regulatory "tail" that contains the key tyrosine 527. When phosphorylated, Y527 acts to hold Src in a closed, inactive state [3$5]$.

Organization of the Src domain structure is important in understanding the ways in which it is regulated in the cell. When inactive, Src exists in a closed conformation that is independent of membrane association. In this closed state, Src maintains several intermolecular interactions to maintain inactivity. These include binding of the $\mathrm{SH} 2$ domain to phosphorylated (p)Y527 and binding of the $\mathrm{SH} 3$ domain to a proline-rich sequence between the $\mathrm{SH} 2$ domain and the kinase domain. The aggregate activity of these interactions helps to provide and maintain stability of the closed confirmation until an activation event occurs [6, 7].

Src activation occurs through multiple mechanisms. The best characterized activation mechanism involves dephosphorylation of pY527 in the C-terminal regulatory tail by the protein tyrosine phosphatase PTP-1B [8]. Dephosphorylation of pY527 abolishes the ability of the $\mathrm{SH} 2$ domain to bind with this portion of c-Src, allowing the 
kinase to unfold and achieve an "open" or activated conformational state. The active state is maintained by pY418 autophosphorylation, which stabilizes the two catalytic lobes of the kinase domain [9]. The active conformation also brings into close proximity two cysteine residues within the SH2 domain (C245) and the kinase domain (C487) that forms a disulfide bond that is also essential for maximal Src kinase activity [10]. A second activation mechanism involves physical disruption of the $\mathrm{SH} 3 / \mathrm{SH} 2$ intramolecular interactions [6]. Target substrates of Src with higher affinity for the SH2 or SH3 domain can out-compete the intrinsic Src interaction sequences for these domains. Such binding allows Src to achieve an open and subsequently active conformation following Y418 phosphorylation as described above [11-14].

Src kinase activity is downregulated by two distinct methods. One mechanism involves "re"phosphorylation of Y527 by c-Src tyrosine kinase (CSK), which restores the $\mathrm{SH} 2$ domain binding site required to maintain the inactive conformation [15]. Cycles of dynamic Y527 dephosphorylation/phosphorylation by PTP-1B and CSK are a critical feature of Src functionality. A second level of regulation involves direct destruction of the Src protein by CBL-mediated ubiquitination of the Src C-terminal region and subsequent proteosomal degradation [16]. This method serves to keep total Src levels in check rather than controlling localized activity throughout the cell.

Utilizing this wealth of knowledge regarding Src regulation, several Src mutants have been created in order to study the specific effects of Src in cellular processes. It is worthy to discuss these scientific tools since they were critical for initially discerning Src function and remain important to this day. Comparison of the v-Src gene product with cSrc homologues indicated a large portion of the C-terminal regulatory region was 
deleted, including Y527. Loss of Y527 results in constitutive Src kinase activity that bypasses downregulation by CSK. Enhanced v-Src activity results in hyperphosphorylation of target substrates and chronic stimulation of downstream signaling pathways important in oncogenic transformation [17]. The dominant nature of v-Src made constructs harboring the coding sequence vital for identifying important downstream substrates involved in tumor initiation and progression. A similar tool based off of $\mathrm{V}$-Src is the Src Y527F mutant [15]. This mutant is also constitutively active due to the inability of Y527 to be phosphorylated, and shares a near-identical spectrum of transforming properties with v-Src [18].

There are also very useful temperature sensitive Src constructs that have been created. These mutants are very useful in that the activity of Src can be controlled by shifting cells expressing these mutants between the temperatures $30^{\circ} \mathrm{C}$ and $37^{\circ} \mathrm{C}$. In this system, $\mathrm{Src}$ is inactive at $37^{\circ} \mathrm{C}$ and active at $30^{\circ} \mathrm{C}$. This allows for complete control of Src activity and allows for very careful observation of the cellular transformation as it manifests due to aberrant Src activity [19].

Other Src mutations have been engineered and used to study the specific contribution of each domain in signal transduction. One example is the mutation of the key arginine (R175) within the $\mathrm{SH} 2$ domain that is required for binding to phosphorylated tyrosine residues [20], as well as the D99N mutation within the $\mathrm{SH} 3$ domain that prevents binding to proline-rich containing sequences [21].

The discussed mutations impact control of Src activation and association with downstream ligands. Conversely, mutation of lysine 298 to methionine (K298M) in the kinase domain prevents ATP binding and renders the Src kinase domain dormant. This 
mutant retains the ability to properly localize and can interact with substrate binding partners, but serves as a dominant-negative protein in that it cannot phosphorylate bound ligands, thus preventing Src-based downstream signaling [22].

\section{Roles of Src in Normal and Cancer Cell Signaling}

Src activity is responsible for regulating a variety of cellular process, including cellular growth, adhesion, and motility. Activation of receptor tyrosine kinases including epidermal growth factor receptor (EGFR) and platelet-derived growth factor receptor (PDGFR) [14, 23] trigger increased Src activity, which in turn phosphorylates downstream proteins that regulate cell cycle and transcription [24]. Src transformation results in increased cyclin D1, E and A expression, as well as an increase in cyclin D1, D3/CDK4/6, cyclin E/CDK2, and cyclin A/CDK2 activity. The CDK inhibitor p27 is also downregulated in Src-transformed cells [25]. The collective effect of increased Srcdriven cyclin activity is sufficient to promote cell cycle progression through the G1 phase into the S phase [25].

Src also controls cell cycle progression through activation of the transcription factor STAT3. STAT3 levels are elevated in v-Src transformed cells [26], promoting anchorage-independent cell growth dependent in part on Src-mediated STAT phosphorylation. Along with Src activity, the activation of the MAP kinase and JNK kinase families phoshorylate the serines on STAT3, allowing for full activation [27]. Src also promotes STAT3 signaling through activation of Shc, which inhibits p53 signaling, an inhibitor of STAT3 [28]. This pathway has also been implicated in EGFR induced 
mitogenesis, especially since it has been shown that Src synergizes with the activation of EGFR and downstream signaling events [29].

Another role Src plays in cellular signaling is the maintenance of cell-substratum and cell-cell adhesions. Src plays an important large role in the turnover of focal adhesions, cytoplasmic adhesion structures that connect the actin cytoskeleton to integrin transmembrane extracellular matrix receptors [30]. Important Src substrates phosphorylated in focal adhesions include focal adhesion kinase (FAK), p190 RhoGAP, and R-Ras [31]. However, the primary role of Src appears to be breakdown of focal adhesions, since Src-null cells are capable of making focal adhesions and maintaining cell-substratum adhesion [32] whereas Src activity, complexed with FAK, is necessary for focal adhesion disassembly $[17,24,31,33]$.

In addition to governing focal adhesion function, Src regulates the adhesiveness of E-cadherin based, cell-cell, adherens junctions by preventing E-cadherin localization and therefore homotypic binding to E-cadherin molecules on neighboring cells [31]. Src also down regulates junctional adhesion through tyrosine phosphorylation of the Ecadherin cytoplasmic domain or through activating phosphorylation of the E3 ubiquitin ligase Hakai, which ubiquitinates E-cadherin to promote endocytic internalization and protesomal degradation [34].

As stated above, Src's role in adhesion breakdown is one of its key functions to promote cellular motility. Dissolution of focal adhesions is an essential step in initiating migration important in such normal process as macrophage extravasation to sites of inflammation, early stages of development, and in would healing [35]. A key signaling pathway in this process is the ability of Src to induce synthesis of the protease calpain. 
Calpain is responsible for the cleavage of FAK and adhesion disassembly. Calpainmediated FAK cleavage releases Src from FAK, allowing Src to phosphorylate additional cytoskeletal proteins necessary for motility [36, 37].

Src also promotes signaling cascades that are necessary for the rearrangement of the actin cytoskeleton that is necessary for cellular movement to occur. In order for cells to migrate, there must be a rapid formation and breakdown of F-actin networks in order to drive the "pushing" of the cellular membrane at the leading edge of the cell during motility. Also, focal adhesions at the rear of the cell must disengage while new adhesions form at the leading edge in order to "grip" the matrix to give the necessary traction for movement [38-40].

In addition to adhesion-based dynamics, Src phosphorylates several key actin regulating proteins responsible for governing actin assembly. One of these proteins is the actin binding protein cortactin. Src phosphorylation of cortactin has been shown to directly impact the efficiency of the formation of branched actin networks at the leading edge of two-dimensional cell movement [41]. Another Src target is p130CAS, which a direct Src substrate and is also downstream of FAK signaling. p130CAS is a multi domain adaptor molecule that binds Src and recruits several proteins essential for actin cytoskeletal organization [42]. It has been shown that p130CAS phosphorylation is implicated in increased tumorigenesis and invasion [43]. Src also controls actin cytoskeleton signaling through activation of the Rho family of GTPases. Src signaling has an antagonistic effect on Rho's contribution to stress fiber formation and the linking of the actin cytoskeleton to adhesions. This effect is mediated by Src phosphorylation and activation of p190RhoGAP. P190RhoGAP in turn binds p120RasGAP, which then 
mediates p120RasGAP dependent activation of RhoGAP. Activated RhoGAP acts directly on active Rho to facilitate GTP hyrolysis, downregulating Rho function to result in stress fiber breakdown [44]. This breakdown of actin filaments in stress fibers is necessary in specific subcellular areas in order for motility to proceed. Src activity also influences activity of the Rho GTPases Rac and Cdc42. Src is necessary for receptor stimulated Rac and Cdc42 dependent signaling events in lamellipodia (flat, actin-rich, ribbon-like protrusions) and filopodia (thin, actin-rich, finger-like projections), respectively [45-47]. This studies collectively point to an essential role for Src kinase signaling in cell migration.

While Src has been studied for nearly a century and a plethora of data have been uncovered concerning its function, it still remains a highly studied oncogene to this day given its central role normal and pathological cell biological processes. 
A.

Src

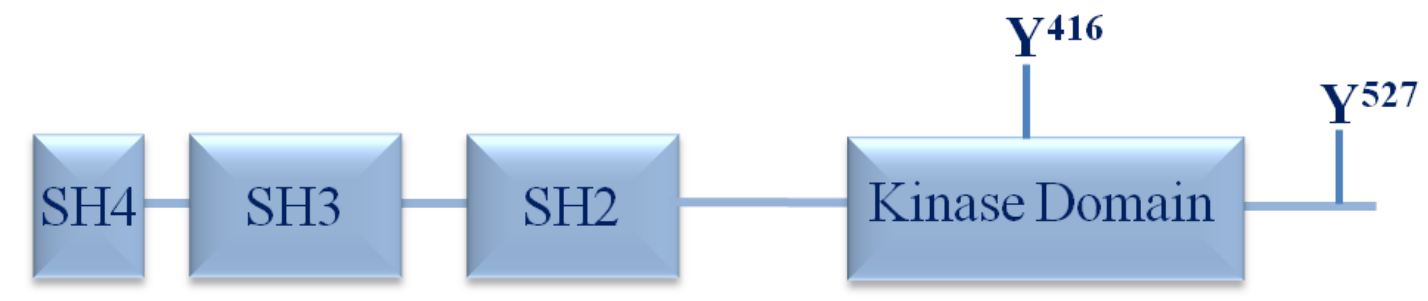

B.

C.

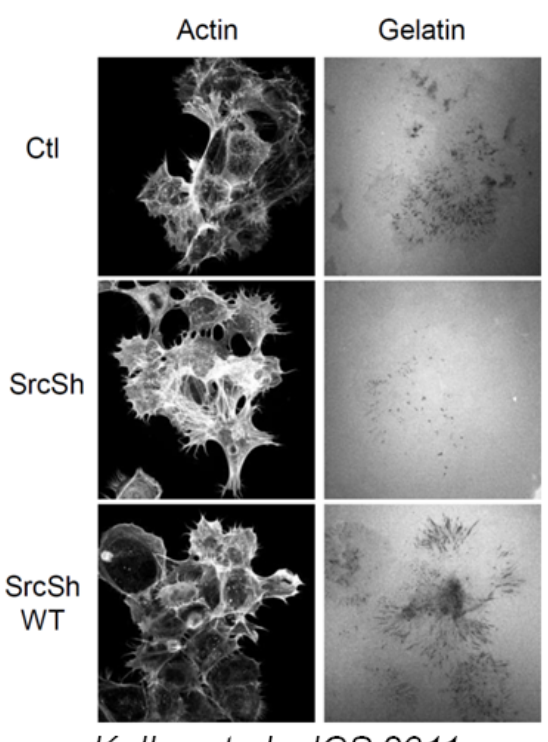

Kelleyet al. JCS 2011

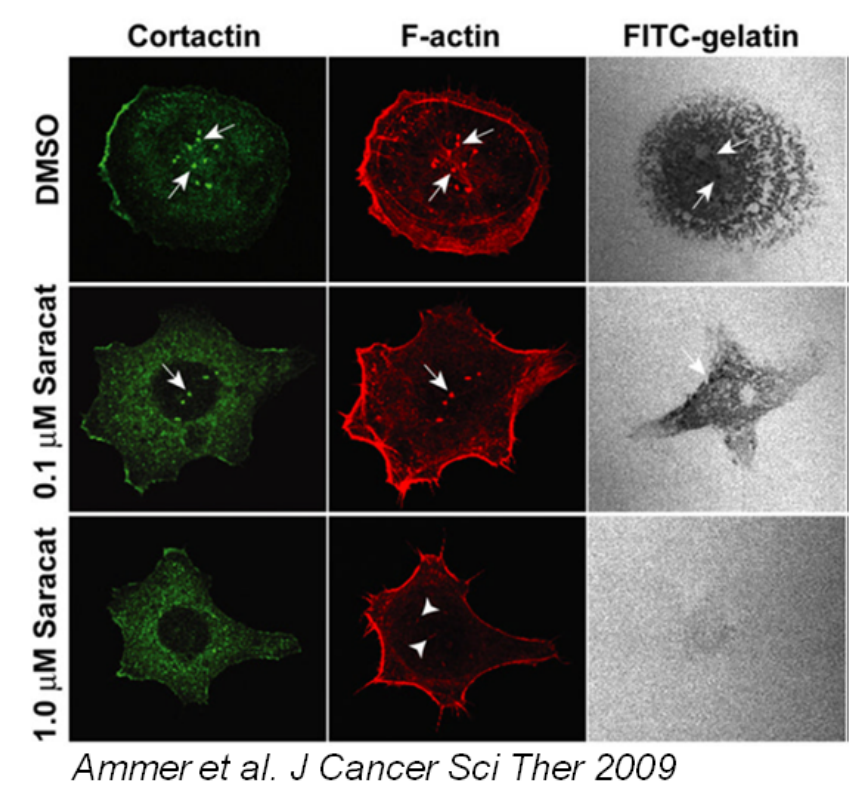

Figure 1: Src kinase structure and role in tumor invasion. (A) Domain structure of Src highlighting the amino acids that are essential to it's function. (B) UMSCC1 cells lose their ability to form invadopodia when Src expression is reduced. (C) UMSCC1 cells lose their ability to form invadopodia upon pharmacologic inhibition of Src kinase activity. 


\section{Cortactin: Integrator of Actin Cytoskeletal Signaling}

Cortactin is a multi-domain [48] actin scaffolding protein and actin nucleation promoting factor that plays an important role in controlling actin dynamics and is a critical component in several actin based cellular structures. Nucleation promoting factors are proteins that assist in the formation, or nucleation, of new actin "daughter" filaments from the existing "mother" filament. This is done through the enhancement of the activity of the actin nucleating protein Arp2/3. Arp2/3's role is to nucleate actin filaments, promoting the formation of newly branching "daughter" filaments from the existing "mother" filament [49]. Arp2/3, once activated, performs this function by a conformation change in the Arp 2 and Arp 3 subunits to mimic actin "barbed" ends, creating new sites of monomer addition to promote de novo filament growth [50, 51]. Cortactin is ubiquitously expressed in mammalian tissue and has a perinuclear intracellular distribution in starved cells. Growth factor or extracellular matrix (ECM) engagement leading to subsequent Rac activation promotes cortactin localization to sites of active cortical actin cytoskeletal growth [52-54]. The key base function of these interactions is the promotion of new branched actin structures through activation of the Arp2/3 complex. The resulting branched actin network provides the necessary tensile strength and protrusive force needed to extend the cell membrane forward during motile and invasive events [55]. Cortactin function is conferred mainly through its interaction with proteins that also play roles in actin dynamics and, conversely, also modulate cortactin function [41]. This scaffolding role is mediated by the diverse array of domains in the cortactin molecule. 
Beginning at the amino terminus, the first cortactin domain encountered is the $\mathrm{N}$ terminal acidic domain (NTA), so named due to the presence of numerous acidic amino acid residues between positions 15 to 35 in the cortactin protein [56]. Notably within this domain is a canonical DDW motif between positions 20-22 that creates an Arp2/3 binding site similar to that found in other Arp2/3 nucleation promoting factor (NPF) proteins [57]. The DDW motif allows cortactin to bind and subsequently activate Arp2/3, directly driving actin nucleation $[50,56]$.

Following the NTA is a region of cortactin containing six complete and one incomplete 37 amino acid tandem repeats (cortactin repeats). [48]. While all repeats share a large degree of sequence homology, the fourth repeat has been shown to be central for F-actin binding, with some contributions from repeats three and five. [56]. The ability of the cortactin repeats to bind F-actin is mediated by lysine residues that form charged patches on the repeat surface. F-actin binding to cortactin can be regulated by acetylation and deacetylation of these lysine patches through the activity of the acetylase P300/CBP-associated factor (PCAF) and the deacetylase histone deacetylase 6 (HDAC6), respectively [58]. This domain also contains a serine residue within repeat 1 (S113) that is a target of the actin regulatory kinase PAK1. However, the functionality of this phosphorylation event has not been clearly elucidated [59]. Together the NTA and cortactin repeats region constitute the amino terminal half of the protein $[41,56]$.

After the repeats region is a predicted alpha-helical domain that contains a 26 amino acid stretch that is a recognition sequence for calpain $[60,61]$. Biochemical work has 
shown that Src phosphorylation of cortactin promotes calpain cleavage, but the biological significance for this is currently unclear.

Carboxyl-terminal to the alpha-helical domain is an unstructured region rich in proline residues (proline-rich region; PRR). This region also contains both serine and tyrosine residues phosphorylated by multiple kinase that are absolutely critical for cortactin function in cell motility and invasion [41]. Serine residues 405 and 418 in the PRR are both targeted by the serine/threonine kinase Erk1/2 and correlate with increased in tumor cell motility and invasion [59,62]. Tyrosine 421,470 , and 486 are targeted by Src and a variety of other kinases, serving as "hot spots" for cortactin regulation [41]. Cortactin tyrosine phosphorylation also results in enhanced tumor cell motility, invasion, and metastasis [62] by creating docking sites for $\mathrm{SH} 2$-containing adaptor proteins and kinases the ultimately regulate increased Arp2/3 nucleation activity, actin polymerization and membrane protrusion [63-65].

Lastly, the extreme $\mathrm{C}$-terminal region of cortactin contains an $\mathrm{SH} 3$ domain that binds a variety of ligands involved in regulating actin dynamics, endocytosis, and non-receptor kinases $[50,66,67]$.

\section{Cortactin Function}

The best characterized and established cortactin function is regulation of Arp2/3based actin polymerization and subsequent membrane protrusion through direct and indirect means. In keeping with a role in actin dynamics, cortactin is a component of several actin-based subcellular structures. Two of these structures, filopodia and lamellipodia, are laterally protrusive motile structures formed by motile cells in two 
dimensional settings [68]. Filopodia are small actin-based, spike-like projections emanating from the cell body that are thought to function as "sensors" of the surrounding cellular microenvironment to promote cell polarity and directed cell movement [69]. Lamellipodia are flat, broad, actin-rich structures with rapid adhesion and actin cytoskeletal turnover that function to pull the cell along a directional trajectory [70]. Cortactin is also a component of actin-based dorsal ruffles, or "waves" that form on the surface of many cell types in response to growth factor stimulation, where they play a role in receptor tyrosine kinase endocytois and subsequent downregulation [71]. On the ventral cell surface, cortactin is a major core component of extracellular matrix degrading structures known as invadopodia. These structures protrude from the cell base into the underlying substratum and secrete matrix metalloproteinases (MMPs) that focally degrade ECM. This action by invadopodia is thought to be a critical mechanism in initiating tumor invasion, allowing cells to cross the basement membrane and invade the underlying stroma, ultimately resulting in metastatic progression [72].

Along with motility-based actin structures, cortactin is a critical component of the actin machinery involved in endocytic downregulation of receptor tyrosine kinases as well as general endo/exocytic vesicle trafficking. Cortactin participates in endocytosis through the binding of the $\mathrm{SH} 3$ domain to the pinchase dynamin 2, coupling dynamin membrane scission activity to the dynamic cortical actin cytoskeleton that surrounds clathrin-coated vesicles. The ability of cortactin to activate and drive Arp2/3 actin polymerization in this process is thought to aid in vesicle formation by initiating membrane deformation prior to membrane severing by dynamin [66, 73-75]. Cortactin remains associated with a vesicle subset, where it is thought to aid in Arp2/3 driven 
intracellular vesicle motility by promoting actin-based propulsion of the formed vesicle through the cytoplasm [76].

The participation of cortactin in several important actin-based cellular processes has led to intense investigation into the molecular mechanisms underlying cortactin regulation of actin binding and NPF activity. In determining how cortactin interacts with F-actin, 3D reconstruction and subsequent analysis of electron micrographs depicting the cortactin repeats region bound to F-actin demonstrate that cortactin interacts with individual actin subunits within F-actin filaments in the same regions as gelsolin and ADF/cofilin [77-79]. Cortactin binding to F-actin deepens the cleft between adjacent actin monomers. This is predicted to impart an inherent instability in the filament, exposing new binding surfaces for Arp2/3 complex or other F-actin interacting proteins [77]. In support of this, a separate study showed that cortactin preferentially binds newly formed actin filaments versus older pre-existing filaments [80].

While the F-actin binding ability of cortactin is key for maximal Arp2/3 filament formation [81], cortactin plays additional roles in Arp2/3-based F-actin network formation besides direct Arp2/3 activation via the NTA domain. Arp2/3 generated filaments by themselves are unstable, breaking apart by a process known as "debranching" to allow individual filaments to be severed and depolymerized [82]. Binding of cortactin to the Arp2/3-F-actin complex stabilizes the branch juncture, making the resulting F-actin networks more resistant to breakdown [81]. These stable actin branches are absolutely critical for proper membrane protrusion and cell motility $[83,84]$. Branch stabilization is achieved by dual binding between the cortactin NTA domain to the Arp2/3 complex and the cortactin repeats binding the F-actin filament [81]. Along with cortactin, neuronal- 
Wiskott-Aldrich Syndrome protein (N-WASp) is another nucleation promoting factor that can bind and activate Arp2/3 at an efficiency $\sim 10$ fold greater than cortactin [51]. NWASp binds F-actin as well as monomeric globular (G)-actin. The ability to bind monomeric actin enhances the process of actin filament nucleation [85]. In biochemical studies, N-WASp and cortactin have a synergistic effect in activating Arp2/3 [81]. However, how both proteins function in concert is unclear and controversial. One potential mechanism is that cortactin and N-WASp interact and activate Arp2/3 at the same time [50]. Another proposed mechanism is that N-WASp initially binds and activates Arp2/3. Cortactin then displaces the N-WASp from pre-activated Arp2/3, binds and holds Arp2/3 in the open confirmation along with maximally stabilizing the branching Arp2/3-F-actin complex [86]. Cortactin also indirectly promotes Arp2/3 activation apart from NTA-mediated interactions with Arp2/3. Cortactin binds N-WASp through its SH3 domain, releasing N-WASp from an auto-inhibited state and promoting localization of N-WASp to sites of active polymerization [87]. The N-WASp binding protein WIP (WASP-interacting protein) also binds the cortactin SH3 domain [88] and assists in promoting cortactin-based N-WASp Arp2/3 nucleation [89]. How specific cortactin complexes with Arp2/3, N-WASp and cortactin are utilized and regulated in cells is an area of active ongoing investigation.

From these studies, it is clear that cortactin has a prominent role in actin cytoskeleton regulation that impacts many cellular processes. As briefly mentioned earlier, phosphorylation of cortactin is a means of controlling its function in governing these events. A main interacting partner of cortactin that is responsible for its regulation is the non-receptor tyrosine kinase Src. In the following section, the history of the 
interplay between these two molecules and their impact on normal and tumor cell signaling will be discussed.

A.

Cortactin

$\operatorname{Arp} 2 / 3$

\begin{tabular}{llllllll|l} 
NTA & 1 & 2 & 3 & 4 & 5 & 6 & Helix P-rich $\mathrm{SH} 3$
\end{tabular}

F-actin

N-WASP

B.

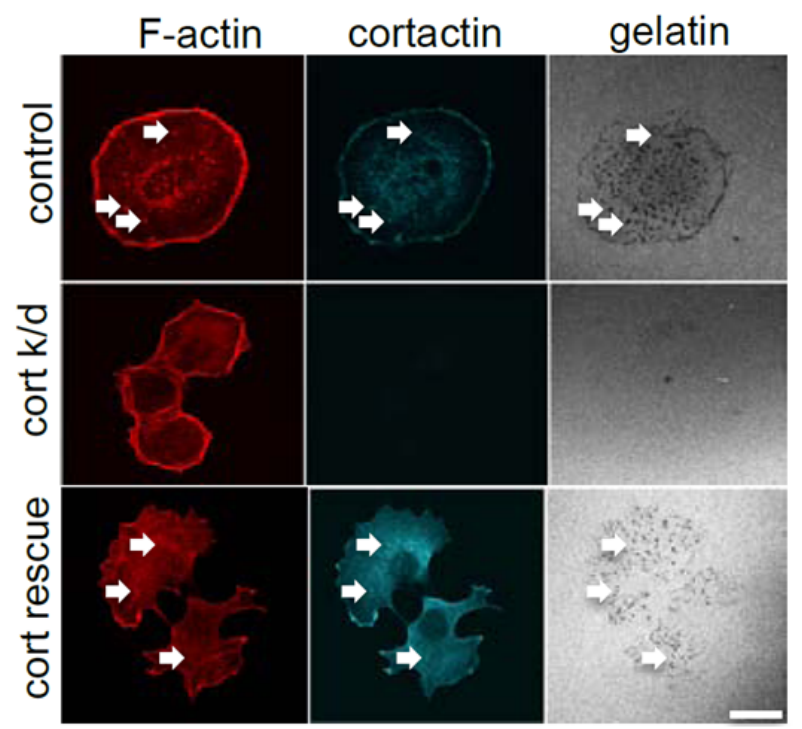

C.

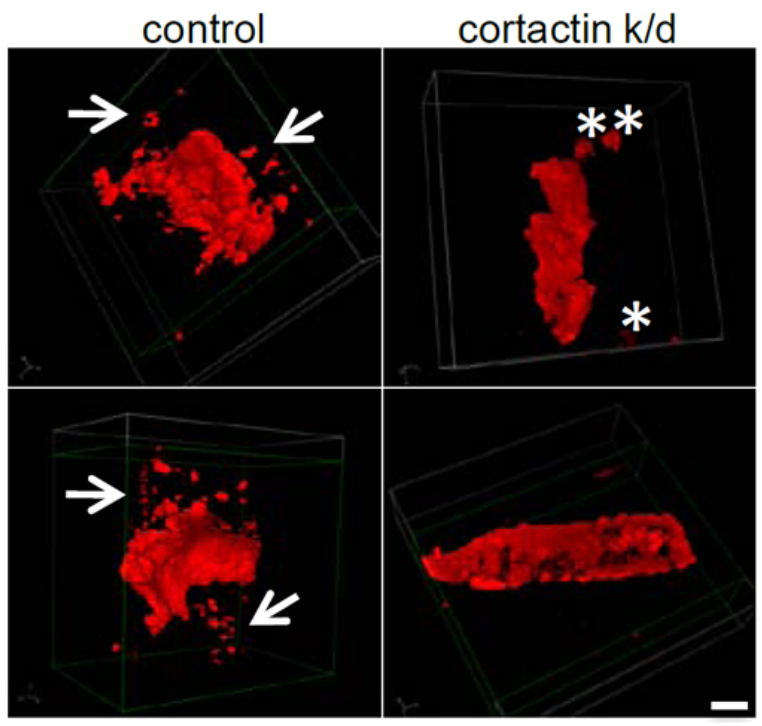

Figure 2. Cortactin structure and role in tumor invasion. (A) Domain structure of cortactin highlighting functionally key tyrosine residues and interacting partners in regulating cytoskeletal dynamics. (B) UMSCC1 cells require cortactin to form invadopodia. (C) Fluorescent labeled UMSCC1 cells were injected orthotopically into a mouse tongue and tumor formation was visualized using two-photon microscopy. 


\section{Src and Cortactin: Phosphoregulation of the Actin Cytoskeleton}

Src has many target substrates that play vital roles in cell movement. Cortactin is one of these substrates that was initially identified along with FAK, CAS, AFAP-110 and paxillin in v-Src transformed cells [90]. Cortactin was initially referred to as p80/85 due to its molecular weight when separated by SDS-PAGE [48]. After cloning and initial studies, it was aptly named "cortactin" due to its newly discovered association with actin filaments and localization at the cell cortex [91]. Activation of the receptor tyrosine kinase fibroblast growth factor receptor-1 (FGFR-1) results in Src activation and enhanced association of Src and cortactin [92]. A later study showed that this pathway activates both MAPK and Src pathways, with MAPK promoting proliferation and Src promoting migration [93], which was confirmed later in a study of FGF-1 signaling in $\mathrm{Src}^{-1-}$ fibroblasts [94]. It was first shown by Okamura et al. that Src directly binds cortactin via its $\mathrm{SH} 2$ domain. This interaction was inhibited by the addition of a pTyr peptide that out competed cortactin for the binding site, indicating that, most likely, the hydrophobic binding pocket of the $\mathrm{Src} \mathrm{SH} 2$ domain is the domain region that interacts with cortactin [95]. However, no additional information regarding the binding mechanism between these two proteins was discussed in this report.

Src-induced tyrosine phosphorylation of cortactin is key in many cellular processes. Vuori et al. showed that Src phosphorylation of cortactin promotes integrin mediated adhesion [96]. Early in vitro studies gave evidence that tyrosine phosphorylation of cortactin by Src inhibits the ability of cortactin to bind and crosslink F-actin filaments [97], in spite of subsequent evidence that cortactin does not function as an actincrosslinking protein [50]. 
Another study showed that the presence of hydrogen peroxide, a reactive oxygen species, induced activation of Src, promoting downstream tyrosine phosphorylation of substrates, including cortactin, as well as actin cytoskeleton rearrangement, membrane blebbing, and cytoplasm shrinkage [98].

With the studies mentioned thus far, it is easy to discern that Src-mediated phosphorylation, when deregulated, can have detrimental consequences in disease states such as cancer, due to alterations in cellular motility. An example of this is work in breast cancer cell lines overexpressing cortactin that have higher incidents of bone metastasis than controls. However, tyrosine phosphorylation null cortactin constructs showed a dramatic reduction in bone metastasis [99]. It is known that overexpression of cortactin and corresponding tyrosine phosphorylation is correlated with increased cell motility and invasion due to amplification of the cortactin gene on the chromosome $11 q 13$ region $[62,100]$.

Rac-induced localization of cortactin is required for Src phosphorylation, where Src phosphorylates cortactin in a processive manner beginning at Y421, indicating that phosphorylation of cortactin by Src phosphorylation is closely tied to the ability of cortactin to regulate actin dynamics. Subsequent biochemical work monitoring Arp2/3 activation assays with N-WASp indicated that Src phosphorylation of cortactin prevented cortactin SH3 domain binding of N-WASp [87], and subsequent Arp2/3 actin nucleation, whereas phosphorylation by Erk1/2 enhanced N-WASp binding and activation [101]. This led to the proposal of a Erk/Src cortactin "switch" that regulated the ability of cortactin to govern N-WASp activity based on the impact of specific kinase phosphorylation events. Central to this theory is that cortactin resides in a "closed" 
conformation with the $\mathrm{SH} 3$ domain unable to bind N-WASp. Phosphorylation of cortactin by Erk1/2 results $\mathrm{SH} 3$ domain release. This allows interaction with N-WASP and subsequent activation of Arp2/3. Src phosphorylation, on the other hand, inhibits the interaction with N-WASP, giving a cyclical regulation "on/off switch" that controls actin polymerization and depolymerization. In spite of the in vitro evidence for this model, it does not reconcile the abundant levels of Src-phosphorylated cortactin at sites of active actin polymerization in cells $[102,103]$. Subsequent in vitro actin assembly assays with Src-phosphorylated cortactin and $\mathrm{SH} 2$ adaptor proteins indicated that the tyrosine residues phosphorylated by Src create docking sites for the $\mathrm{SH} 2$ domain of the adaptor protein Nck1, which in turn binds WIP and N-WASp to facilitate Arp2/3 activation [89]. The creation of a phospho-cortactin/Nck1/N-WASp tripartite complex has been confirmed within invadopodia in invasive breast cancer cells [103], raising the creditability of this mechanism. Additionally, work from our laboratory indicates that cortactin molecules can be serine and tyrosine phosphorylated, further arguing against the simple "on/off" switch mechanism [104, 105].

Functional interplay between Src and cortactin phosphorylation has been most extensively studied with respect to the formation and function of tumor cell invadopodia [106]. Invadopodia and related higher-order structures termed podosomes rely on Src activation and cortactin for their formation and function. Src activation is an absolute requirement for invadopodia formation [107]. Cortactin phosphorylation is increased on Y421 and Y470 within podosomes in multiple cell types [108-110] and within invadopodia [111, 112]. Invadopodia assembly undergoes specific stages that result in the seeding, assembly and acquisition of proteolytic activity. Pre-invadopodia formation 
downstream of Src activation requires cortactin and F-actin to form [111], whereas dynamic cycles of Src activation and cortactin phosphorylation/rephosphorylation are responsible for the "maturation" of pre-invadopodia into invadopodia capable of degrading ECM $[113,114]$. The inability of cells to properly regulate cortactin tyrosine phosphorylation directly contributes to impaired invadopodia degradative function, as evidenced through the use of point mutant and deletion constructs of both proteins [103, $114]$ as well as the drugs targeting Src kinase activity $[107,115]$.

In addition to facilitating the formation of Nck1/N-WASp complexes to drive Arp2/3 nucleation activity, Src-phosphorylated cortactin directly contributes to invadopodia formation through the release of the actin severing protein cofilin, which cuts preexisting F-actin to increase the number of barbed ends to allow increased monomer addition and filament growth [65]. Cofilin activity is regulated by intracellular $\mathrm{pH}$ levels, and recent work has shown that Src-phosphorylated cortactin recruits the $\mathrm{Na}+/ \mathrm{H}+$ exchanger NHE1 to preinvadopodia, initiating cofilin activation through local increases in $\mathrm{pH}[116]$.

In addition to cell motility and podosome/invadopodia formation, Src phosphorylation of cortactin is also involved in regulating several additional cellular processes. Several groups have shown that Src-phosphorylated cortactin is necessary for the internalization of bacterial pathogens as well as their subsequent intracellular motility. [117-120]. Other important cellular functions that require Src phosphorylation by cortactin include neurite outgrowth [121], leukocyte transmigration [122], receptor mediated endocytosis [123], cell injury response [124], cell shrinkage [125], and vascular formation [126]. 


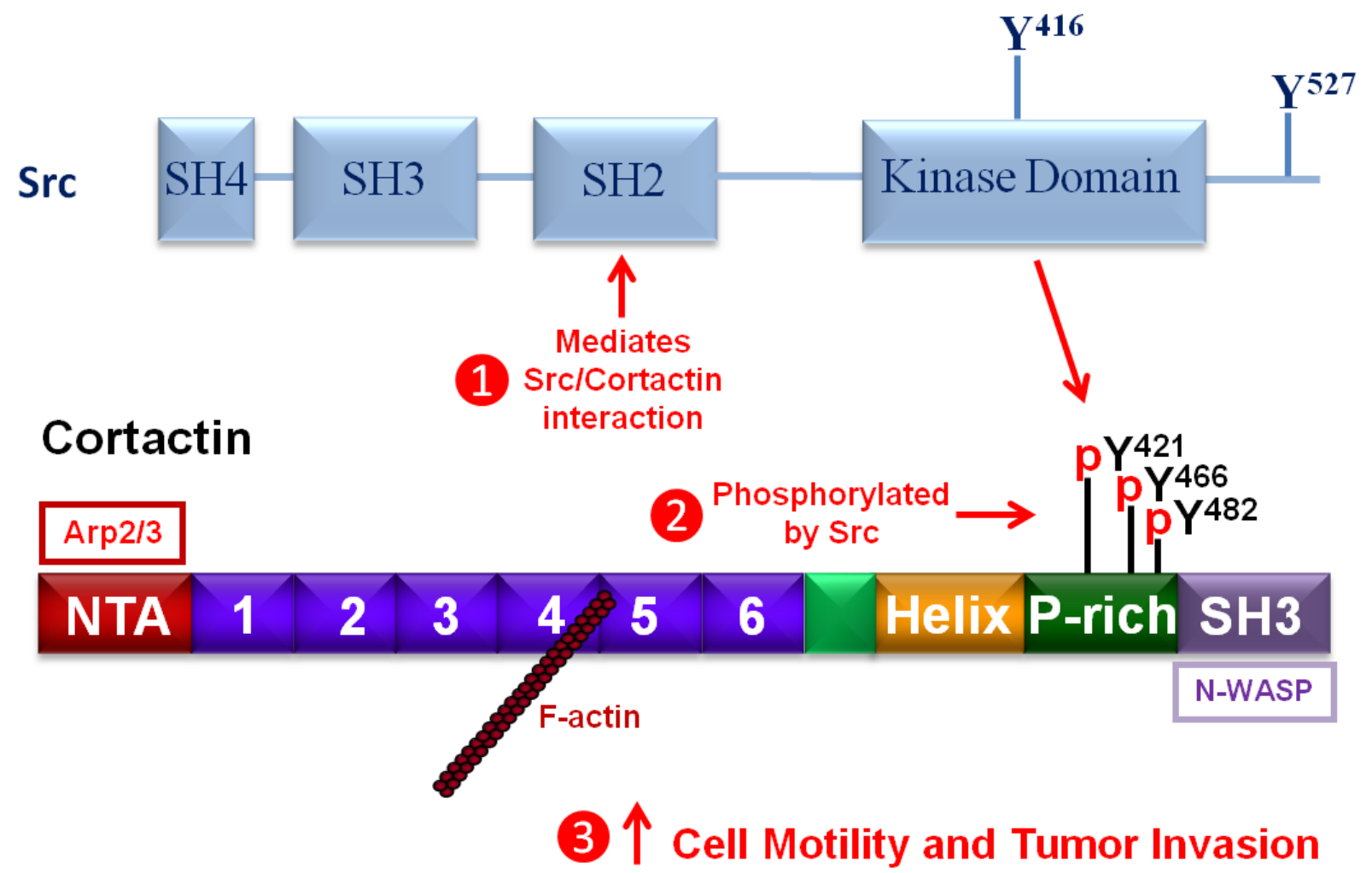

Figure 3. Src and cortatin work in concert to regulate cell motility and invasion. Diagram outlining how Src phosphorylation of cortactin tyrosine residues 421,466 , and 482 plays a role in the regulation of cellular motility and invasiveness. 


\section{SH2 Domains: Non-Conventional Interactions}

Proteins generally consist of specialized domains that are intrinsic to overall function. One of the first discovered and best characterized protein domains is the $\mathrm{SH} 2$ domain. The SH2 domain was first discovered by Sadowski et al. when the sequence of noncatalytic domains of cytoplasmic tyrosine kinases were compared to $\mathrm{Src}$ [3]. SH2 domains consist of approximately 100 amino acids, forming a generally conserved structure consisting of an anti-parallel $\beta$-sheet located between two $\alpha$-helices that are interconnected by four loop structures $[127,128]$. SH2 domains are protein interaction domains that are critical for propagating cellular signaling networks within cells. Recent work by Liu et al. show that $\mathrm{SH} 2$ domains are evolutionarily conserved from the simplest of organisms to the most complex and this conservation has co-developed with the multitude of complex signaling cascades seen in eukaryotic cells [129]. Interestingly, this study also showed that the prevalence of $\mathrm{SH} 2$ domains increased with the increase in complexity of the organisms in which they are found, supporting their role in higher order signaling networks. SH2 domains primarily function by binding to phosphorylated tyrosine residues with specific flanking amino acid sequences in target proteins [130]. These interactions are relatively weak so that they are easily reversible, which is essential for rapid responses to the ever-changing intracellular microenvironment [127]. $\mathrm{SH} 2$ domains contain a hydrophobic binding pocket in which the phosphorylated target tyrosine binds. Binding of phosphotyrosine to the domain is largely mediated by Van Der Waal interactions between the negative oxygen moieties of the tyrosine phosphate and a positively charged arginine residue. This arginine lies within the highly conserved FLVRES sequence present in most $\mathrm{SH} 2$ domains. The arginine is critical for phospho- 
tyrosine binding, as mutagenesis of this residue to lysine or leucine prevents $\mathrm{SH} 2$ domains from binding target phosphotyrosines. The pTyr residue is largely responsible for the binding affinity, while residues within positions -2 to +4 from the tyrosine residue confer specific domain-ligand binding specificity [131].

Although SH2 domains are predominantly known for binding phosphorylated tyrosine residues, there have been many studies published over the past decades that describe non-conventional, phosphotyrosine-independent mechanisms of binding. These studies range from identifying phosphotyrosine independence to determining the precise amino acids responsible for the interaction and encompass a wide variety of protein classes. While the phosphotyrosine-dependent nature of $\mathrm{SH} 2$ domains has been well studied and reviewed, phosphotyrosine-independent interactions have not been categorically assessed in the literature. This chapter will examine the lesser renowned modes of phosphotyrosine independent SH2 domain interactions with their target ligands.

\section{Phosphotyrosine-independent Regulation of Global SH2 Domain Function}

Initial studies into how $\mathrm{SH} 2$ domain ligand binding is regulated was discovered by Rameh et al. in that PI3K lipid products can directly bind $\mathrm{SH} 2$ domains and interfere with their ability to bind tyrosine-phosphorylated proteins. Initial results of this study showed that treatment with PI3K inhibitors stabilized the association of PI3K with tyrosine-phosphorylated proteins and that overexpression of PI3K reduced these interactions. These results suggested the PI3K lipid products interfere with the ability of $\mathrm{SH} 2$ domains and pTyr-containing proteins. Subsequent work showed direct lipid binding to all tested SH2 domains (p85 subunit of PI3K (N-terminal and C-terminal), Abl, 
Lck, and Src). The phospholipid PtdIns-3,4,5- $\mathrm{P}_{3}$ showed competitive inhibition with pTyr peptides in this study, indicating that $\mathrm{SH} 2$ binding to target ligands is affected by the balance of lipid and tyrosine phosphorylated protein binding sites present in the cytoplasm. Interestingly, the key arginine residue necessary for phosphotyrosine binding in the $\mathrm{Src} \mathrm{SH} 2$ domain was not required for lipid binding, indicating different charged residues in the hydrophobic binding pocket facilitate the interaction. Biological evidence was demonstrated by showing that binding of the PI3K p85 subunit C-terminal $\mathrm{SH} 2$ domain to tyrosine phosphorylated insulin receptor was blocked by Ptdlns-3,4,5$\mathrm{P}_{3}$. Overall, this study was the first to show that $\mathrm{SH} 2$ domains bind the lipid products of PI3K [132].

Substrate recognition is a critical function of $\mathrm{SH} 2$ domains. Typically, this consists of the recognition of a phosphorylated tyrosine residue and its flanking amino acids. However, it has been shown that more determinants are required than just simple sequence recognition. Work by Bae et al. identified a secondary site on $\mathrm{SH} 2$ domains responsible for their target selectivity separate from the hydrophobic binding pocket utilized in phosphotyrosine-dependent interactions. This site does not require ligand tyrosine phosphorylation for binding, but rather utilizes a series of charge-based interactions. Crystallography and NMR-based binding studies with the PLCy N-SH2 and $\mathrm{C}-\mathrm{SH} 2$ domains to specific pTyr binding sites on FGFR1 identified this new region, which was subsequently termed the "specificity pocket" and is found in all SH2 domains, providing additional means to reinforce domain-ligand specificity during downstream signaling events [133]. 


\section{Phosphotyrosine-independent SH2 Domain Interactions: SH2 Domain Only Proteins}

Some proteins consist entirely of a single $\mathrm{SH} 2$ domain. These single domain proteins act as modulators of signaling pathways by binding to their targets to prevent binding to the same site by other proteins. One example of this type of protein is signaling lymphocyte-activation molecule (SLAM)-associated protein (SAP or SH2D1A). SAP is a T-cell specific protein that is the product of a gene mutated in X-linked lymphoproliferative syndrome (XLP). It has been shown to bind SLAM (CD150) on its cytoplasmic tail and inhibit the recruitment of the $\mathrm{SH} 2$ domain containing phosphatase SHP-2. SAP was predicted to recognize Y281 of SLAM, but binding was found to occur regardless of the Y281 phosphorylation state. However, phosphopeptides mimicking this binding site were able to block the interaction, showing that the hydrophobic binding pocket of the $\mathrm{SH} 2$ domain is necessary to mediate binding [134]. Subsequent studies determined that the SAP SH2 domain recognizes a T-I-Y-X-X-(V/I) motif that occurs twice in SLAM. The interesting aspect of this motif is that SH2 recognition extends $\mathrm{N}$ terminally and C-terminally from the tyrosine residue, where these extended regions mediate SAP binding to SLAM with affinities similar to SH2-pTyr ligand interactions $[135,136]$

EWS-FLI1-activated transcript 2 (EAT2) is another protein that consists of a single $\mathrm{SH} 2$ domain that may also be capable of phosphorylation-independent binding. Poy et al. stated in unpublished data that EAT2 is able to bind non-phosphorylated peptides and recognizes similar motifs as SAP [135]. However, Morra et al. showed that EAT2 requires tyrosine phosphorylation of SLAM before it can bind, unlike SAP. The structural and functional similarities of EAT2 to SAP it makes it likely that EAT2 
contains the ability to bind substrate ligands in a phosphotyrosine-independent manner, regardless of its ability to bind nonphosphorylated SLAM [137].

\section{Intramolecular SH2 Domain Interactions}

SH2 containing proteins are subject to regulation by forming intramolecular interactions with peptide sequences within the parent protein. One such mechanism is seen within the protein tyrosine phosphatase SH-PTP2. It was shown that the SHPTP2 SH2 domains bind to the catalytic domain of the protein, and kinetic studies showed that non-phosphorylated, bacterially expressed SH-PTP2 was negatively regulated by this interaction [138]. Another example was observed in the non-receptor tyrosine kinase IL2-inducible T-cell kinase (Itk). This study showed that following activation, Itk undergoes autophosphorylation of tyrosine 180 within its SH3 domain. In order for this to occur, the SH2 domain of Itk must bind its kinase domain. The phosphotyrosine-independence of this interaction was determined by the fact that the Itk $\mathrm{SH} 2$ domain could interact with the bacterially produced full length Itk moleucule. This group also showed that mutating R265 in the Itk SH2 domain to alanine, which eliminates its ability to interact with phosphotyrosine motifs had no deleterious effects on its ability to bind the Itk kinase domain, confirming the phosphotyrosine independence of this interaction [139].

Src also undergoes phosphotyrosine independent intramolecular $\mathrm{SH} 2$ domain regulation thought to regulate Src activation. Using phosphorylation of FAK as a readout, it was demonstrated that $\mathrm{C} 245$ in the $\mathrm{SH} 2$ domain and $\mathrm{C} 487$ of the kinase domain do not undergo oxidation upon cell adhesion or $\mathrm{H}_{2} \mathrm{O}_{2}$ treatment as expected. 
Mutation of either cysteine prevented FAK phosphorylation, indicating that C245/467 are vital to redox-activated Src activation. These data are suggestive of disulfide bonding between C245 and C467 to stabilize Src in an active conformation, but evidence of cystine bonding between these two residues was not directly shown [10].

Linking of a tyrosine kinase's $\mathrm{SH} 2$ domain to its own kinase domain is emerging as a common feature in regulating kinase activity and kinase substrate recognition. The $\mathrm{SH} 2$ domains and kinase domains of Fes and Abl contain linkages that regulate protein function. This study used elegant crystallographic analysis to identify these interactions. In the case of Fes, a stable unit forms due to a polar interaction between the $\mathrm{N}$-terminal helix $\alpha \mathrm{A}$ region of the $\mathrm{SH} 2$ domain and the catalytically important helix $\alpha \mathrm{C}$ region of the kinase domain. These residues at the SH2-kinase interface stabilize the active Fes conformation by correctly positioning and stabilizing the kinase helix $\mathrm{aC}$ region, which forms the active site. This study also showed Abl kinase activity is regulated by a similar binding event. The Abl SH2 domain forms a polar interaction with the $\mathrm{N}$-lobe of the kinase domain. As with Fes, this interaction is required for efficient Abl activity and substrate phosphorylation [140].

\section{Lipases}

Lipases play an important role in the propagation of receptor mediated cell signaling. Hydrolysis of the membrane phospholipid, phosphatidylinosiol 4,5-bisphosphate, is important in regulating the interaction of many hormones and growth factors with their cell surface receptors. Catalyzation of this reaction is mediated by phosphoinositidespecific phospholipase C (PLC) isozymes. This produces two intracellular messengers, 
diacylglycerol and inositol 1,4,5-trisphosphate $\left(\mathrm{IP}_{3}\right)$, which activates protein kinase $\mathrm{C}$ and releases intracellular $\mathrm{Ca}^{2+}$, respectively. Specific kinase and phosphatase activity further breaks down $\mathrm{IP}_{3}$ into other inositol phosphates which play roles in intracellular signaling [141].

PLC-y1 plays critical roles in both cellular proliferation and differentiation [142, 143]. It is activated by tyrosine phosphorylation [144], with modulation of this activity also being dependent upon serine/threonine phosphorylation [145, 146]. PLC-y1 contains two $\mathrm{SH} 2$ domains and a SH3 domain that are responsible for substrate binding. These $\mathrm{SH}$ domains are essential for cell proliferation and growth factor-induced mitogenesis, although the exact mechanisms remain unclear [28]. PLC-y1 has also been shown to be elevated in cancer, implicating it in a role in tumor progression [147].

It has been shown by two groups that the C-terminal SH2 domain of PLC-y1 can interact with substrates independently of tyrosine phosphorylation. Muller et al. examined a set of $\mathrm{SH} 2$ domain proteins for their ability to bind separately the BCR and Abl components of the BCR-Abl fusion protein. A subset of the $\mathrm{SH} 2$ domains bound non-tyrosine phosphorylated BCR, including the PLC- 1 1 C-terminal SH2 domain, but the specific binding mechanism was not evaluated [148]. Joseph et al. also reported another instance in which the PLC- $\mathrm{y} 1 \mathrm{C}$-terminal SH2 domain can bind to a substrate in a phosphotyrosine-independent manner. PLC- $\mathrm{Y} 1$ is phosphorylated at $\mathrm{Y783}$ by the non-receptor tyrosine kinase Itk following $T$ cell receptor activation. This phosphorylation event activates PLC- $\mathrm{Y} 1$ lipase activity. For this phosphorylation to occur, the C-terminal SH2 domain of PLC- $\mathrm{y} 1$ must bind to the kinase domain of Itk. Phosphotyrosine independence was determined by mutating R694 and R696 in the 
PLC- $\mathrm{y} 1 \mathrm{SH} 2$ domain to alanine (which abolishes phosphotyrosine-dependent binding). These mutations had no effect on the ability of PLC- $y 1$. Follow-up work from the same group (Min et al.) utilized molecular modeling and NMR to elucidate the exact determinants of the phosphotyrosine-independent interaction between the PLC- $\mathrm{Y} 1 \mathrm{SH} 2$ domain and Itk. They found that the site of interaction with the PLC- Y1 C-terminal SH2 domain lies outside of the phosphotyrosine binding pocket. The residues responsible for binding included $\mathrm{K} 711$ and E709 on the CD loop of the $\mathrm{SH} 2$ domain and a basic patch C-terminal to the binding pocket consisting of R748, R749, K751, and R753. Non-conformational mutations of these residues to alanine effectively abolished the interaction of the SH2 domain with Itk and the subsequent phosphorylation of $\mathrm{Y} 783$ by Itk. This study also showed that the PLC- $\mathrm{Y} 1 \mathrm{C}$-terminal $\mathrm{SH} 2$ domain retains the ability to bind its canonical ligands while interacting with Itk in this phosphotyrosine independent manner, indicating that increased complexity is found in select $\mathrm{SH} 2$ domain-ligand interactions [139].

\section{G-protein Regulators}

Guanine nucleotide exchange factors (GEFs) and GTPase activating proteins (GAPs) are two protein classes responsible for regulating proteins with GTPase activity. GTPases cycle between an active (GTP bound) and inactive (GTP unbound) state. This cycling is activated by GEFs by their promotion of the exchange of GDP for GTP and the activity is terminated by GAPs, which promote the hydrolysis of GTP to GDP [149]. Close regulation of GTPases are crucial considering the roles they play in 
diverse processes such as cell migration, vesicle trafficking, cell survival, and cell cycle progression $[149,150]$.

Subsets of GEFs and GAPs contain SH2 domains to aid in specifying ligand recognition, aiding in proper regulation of GTPase activity. Some SH2-containing GEFs and GAPs bind ligands in a phosphotyrosine-independent manner. The GEF Vav1 constitutively interacts with the receptor tyrosine kinase Mer through a phosphotyrosineindependent interaction that regulates Vav1 subcellular localization and downstream Rho family GTPase activity [151]. The GTPase-activating protein (GAP) contains two $\mathrm{SH} 2$ domains, and the $\mathrm{N}$-terminal domain is capable of binding to the non-tyrosine phosphorylated BCR segment of the BCR-Abl fusion protein. Interestingly, the GAP Cterminal $\mathrm{SH} 2$ domain was not able to bind to the BCR protein, suggesting that these two $\mathrm{SH} 2$ domains possibly function to regulate different subsets of ligands. [148].

\section{Adaptor Proteins}

Adaptor proteins play an important role in propagating signals in cellular pathways by bridging catalytic proteins to their specific substrates. Adaptor proteins can also serve as substrates, whereby tyrosine phosphorylation creates binding sites for additional signaling molecules. Adaptor proteins also serve as scaffolds by holding macromolecular complexes together, such as focal adhesions [152]. Like the other aforementioned protein classes, certain $\mathrm{SH} 2$ domain-containing adaptor proteins bind target proteins in a phosphotyrosine-independent manner.

The growth factor receptor-binding protein (Grb) family of adaptor proteins serve to bridge proteins important in transmitting intracellular signals. Grb proteins share a 
similar structure, having a proline-rich $\mathrm{N}$-terminal region, a Ras-associating-like domain, a pleckstrin homology domain, a BPS domain specific to the Grb family, and a Cterminal SH2 domain $[152,153]$ In order to study binding specificity, the Grb2 SH2 domain was screened against a phage display library of $10^{7}$ different peptide sequences and 18 binding peptides were identified that all had identical sequences. The peptide is referred to as G1 and is a 9-mer peptide containing a single tyrosine residue flanked by two cysteine residues that can form a disulfide bond to stabilize the peptide in a cyclic conformation. This peptide binds the Grb2 $\mathrm{SH} 2$ domain in a phosphotyrosineindependent manner, and contains a Tyr-X-Asn (YXN) motif similar to other Grb2 SH2 domain phosphotyrosine ligands. The G1 peptide requires an intact disulfide bond between the flanking cysteine residues, since reduction with dithiothreitol (DTT) diminishes binding. The $\mathrm{G} 1$ peptide inhibits binding of the $\mathrm{Grb} 2 \mathrm{SH} 2$ domain to the ligand Shc, as well as preventing binding of short phospho-tyrosine peptides, suggesting that $\mathrm{G} 1$ binds in the hydrophobic binding pocket of the Grb2 SH2 domain. When introduced into cells, non-reducible thioester G1 derivatives prevent Grb2 SH2 binding to cellular targets, confirming that Grb2 phosphotyrosine independent binding occurs in within the cytoplasm [154].

A parallel study evaluating binding partners for the $\mathrm{Grb} 7 \mathrm{SH} 2$ domain indicated that peptides with a YXN motif interacted independent of tyrosine phosphorylation. Mutation of the $\beta D 5$ and $\beta D 6$ positions in the Grb7 SH2 domain to residues present in the Grb14 SH2 domain blocked binding of YXN peptides, as well as to pY1139 on the receptor tyrosine kinase ErbB2, a known Grb7 interacting partner. YXN peptides were able to compete with Grb7 binding to tyrosine phosphorylated ErbB2, demonstrating that a non- 
phosphorylated peptide could out-compete a known phosphotyrosine target of the Grb7 SH2 domain [155].

Grb10 is another Grb family member that utilizes $\mathrm{SH} 2$ domain phosphotyrosineindependent binding. Yeast two-hybrid screening indicated that the Grb10 SH2 domain binds the Erk1/2 activating kinases Raf1 and MEK. Binding to MEK1 requires phosphorylation of threonine 386 and the presence of the key phosphotyrosine-binding arginine (R520) in the Grb10 SH2 domain, indicating that other phosphorylated amino acids in select instances can substitute for phosphotyrosine in mediating $\mathrm{SH} 2$ domain docking [156].

A series of separate studies determined that the Grb10 SH2 domain also mediates protein-protein interactions outside of the phosphotyrosine binding cleft. Binding of the Grb10 SH2 domain to Nedd4 (neuronal precursor cell-expressed developmentally down-regulated 4) occurs in a phosphotyrosine manner [157], utilizing three separate charged patches between the Grb10 SH2 domain and Nedd4 determined by NMR of the Grb10 SH2-Nedd4 complex [158]. These studies raise the potential for select SH2 domains to bind multiple ligands through the use of sequences within and external to the phosphotyrosine binding cleft.

The tensin family of adaptor proteins plays roles in the formation of focal adhesions. Generally the structure of this family consists of a central region necessary for protein function, such as F-actin binding with Tensin1, and then an SH2 domain followed by a phosphotyrosine binding domain (PTB) that interacts with integrin cytoplasmic tails [159]. The family member cten (C-terminal tensin like) differs in that it has a smaller molecular mass and only shares homology with other family members through its $\mathrm{SH} 2$ 
and PTB domains. Cten has been show to be absent in prostate cancer, suggesting a role as a tumor suppressor [160]. One role discussed for this function of cten is its interaction with the protein DLC-1 (deleted in lung cancer-1). Binding to DLC-1 is mediated by the cten SH2 domain through pTyr independent means that utilizes the key arginine in the hydrophobic binding pocket (R474). DLC-1 contains a motif (SIYDNV) that is similar to the SAP SH2 binding site on SLAM. Using this information, point mutants in the DCL-1 SIYDNV region analogous to required residues on SLAM tyrosine ablate cten $\mathrm{SH} 2$ binding, indicating that these proteins share similar $\mathrm{SH} 2$ binding motifs. [161]. Similar studies conducted with the related protein tensin2 indicated that the tensin2 $\mathrm{SH} 2$ domain binds to the same SIYDNV region on DLC-1, where NMR studies suggest that tyrosine 41 within the $\mathrm{SH} 2$ domain was important for mediating phosphotyrosine independent binding to the DCL-1 sequence [162].

The adaptor protein suppressor of cytokine signaling-1 (SOCS1) regulates Vav1 GEF activity through binding of the SOCS1 SH2 domain to Vav1. While this binding was shown to be independent of Vav1 tyrosine phosphorylation, this study did not determine the precise mechanism utilized in tyrosine phosphorylation independent binding [163]. In addition to SOCS1, the SH2 domain of the adaptor protein Shc also interacts with proteins independent of tyrosine phosphorylation. Shc plays roles in linking activated cell surface receptors to Ras signaling following receptor tyrosine kinase activation. Shc proteins contain a C-terminal SH2 domain, a glycine/proline rich domain, and an N-terminal PTB domain. The novel Shc SH2 domain binding partner Protein Activating in Lymphocytes (mPAL) binds Shc without being tyrosine phosphorylated, utilizing the key phosphotyrosine binding arginine (R397) on Shc to 
mediate mPAL binding. This study thus demonstrated that binding of the Shc SH2 domain to MPAL requires some of same molecular constituents as phosphotyrosine binding [164].

\section{$\underline{\text { Transcription Factors }}$}

Transcription factors are proteins that bind to specific DNA sequences and control RNA production within the cell. They can act alone or in a complex as either activators or repressors of gene transcription. The signal transducers and activators of transcription (STAT) proteins are a family of seven proteins that function by relaying signals from membrane receptors to the nucleus, where they regulate gene transcription. One member of this family, STAT3, is a master regulator of gene transcription. Structurally, STAT3 contains an N-terminal coiled-coiled domain, a DNA binding domain, an $\mathrm{SH} 2$ domain, and a C-terminal transactivation domain. The primary function of the $\mathrm{SH} 2$ domain is to bind to phosphotyrosine residues on activated transmembrane receptors $[165,166]$. However, the STAT3 SH2 domain also participates in phosphotyrosine-independent interactions by binding to the p32 subunit of replication protein A (RPA). This interaction augments the phosphorylation of STAT3 upon EGF stimulation. While the mechanism of how this is achieved is not clear and was not further investigated, it was shown that STAT3 retains a higher level of phosphorylation when interacting with p32 RPA, resulting in increased transcriptional activity. 


\section{$\underline{\text { Kinases }}$}

Protein kinases act on proteins to modify their activity by transferring phosphate groups from ATP to specific substrates. The main function of kinases is to transmit signals in order to control a variety of complex signals in cells. Two subsets of protein kinases are lipid and tyrosine kinases, which are named for the small molecule substrates they phosphorylate. These two kinase groups have members that utilize non-conventional $\mathrm{SH} 2$ domain interactions.

An important lipid kinase in cellular signaling is phosphoinositide 3-kinase (PI3K). It has been shown to play key roles in cell survival, proliferation, differentiation and cancer. PI3K propagates cellular signaling from growth factors and cytokines into intracellular messages by phosphorylating phosphatidylinositol 4,5 bisphosphate $\left(\mathrm{PIP}_{2}\right)$ at the 3 position of the inositol ring, producing phosphatidylinositol 3,4,5-triphosphate $\left(\mathrm{PIP}_{3}\right) . \mathrm{PIP}_{3}$ functions as a second messenger, activating the serine kinase Akt and other downstream pathways that control the cellular events mentioned above. PI3K is a heterodimer that is made up of a p110 catalytic subunit and a p85 regulatory subunit that controls binding, activation and localization $[167,168]$. The p85 subunit contains $\mathrm{N}$ terminal and $\mathrm{C}$-terminal $\mathrm{SH} 2$ domains that are responsible for protein interactions and these domains have been implicated in phosphotyrosine-independent interactions.

The Anderson laboratory has extensively studied the interaction of the $\mathrm{N}$-terminal and $\mathrm{C}$-terminal $\mathrm{SH} 2$ domains of the p85 subunit of PI3K with the Raf isoform A-Raf. Initial work using phage display to identify binding partners to a section of basic residues in A-Raf that bound the A-Raf $\mathrm{SH} 2$ domain identified both $\mathrm{PI} 3 \mathrm{~K}$ SH2 domains as capable of binding to A-Raf independent of A-Raf tyrosine phosphorylation. The 
common theme between the phage display A-Raf peptides is that they all contained the same core motif of (L/I)-A-(R/K)-I-R. A-Raf has four of these sequences (LIKGRK, LQRIRS, EQRERK, and DKKKVKNL) and both p85 SH2 domains can bind all four of these regions. Typically the $\mathrm{p} 85 \mathrm{SH} 2$ domains recognize $\mathrm{pY}-\mathrm{X}-\mathrm{X}-\mathrm{M}$ ligands. This study shows that both p85 domains can interact with several basic-X-basic and led the authors to conclude that the site of $\mathrm{p} 85 \mathrm{SH} 2$ domain binding is separate from the hydrophobic binding pocket necessary for phosphotyrosine binding [169]. Follow up work by the same group indicated that mutation of the key arginines in the p85 Nterminal (R358) and C-terminal (R649) SH2 domains had no effect on their ability to bind A-Raf. A series of mutational studies on A-Raf, along with phosphatase treatment and phosphotyrosine peptide competition experiments resulted in the conclusion that that there are two separate binding sites for A-Raf on the PI3K SH2 domains, both of which are phosphotyrosine independent. The first binding site overlaps the region of the $\mathrm{SH} 2$ domain responsible for phosphotyrosine binding, since a phosphopeptide based on the p85 PDGFR docking site could partially block SH2 domain binding. This is termed the phosphotyrosine-dependent binding site (PDB). The second site lies in an area away from the PDB and does not overlap. This was referred to as the phosphorylation-independent binding site (PIB) [170].

Tyrosine kinases occur as transmembrane receptor and cytoplasmic non-receptor tyrosine kinases. An important group of non-receptor tyrosine kinases are the $A B L$ family of proteins. $\mathrm{ABL}$ proteins contain an $\mathrm{SH} 3, \mathrm{SH}$, and catalytic domain that is typical of many cytoplasmic tyrosine kinases. $A B L$ also has a DNA biding domain that plays a nuclear role in DNA repair [171]. The $\mathrm{ABL}$ SH2 domain binds several 
substrates in a phosphotyrosine-independent manner in the BCR-ABL and v-ABL variants.

Sequences in BCR necessary for transformation by the BCR-Abl oncogene bind to the $\mathrm{Abl} \mathrm{SH} 2$ domain in a phosphotyrosine-independent manner. The Abl $\mathrm{SH} 2$ domain binds to two serine/threonine rich regions in the $\mathrm{BCR}$ protein in a phosphoserine/threonine dependent manner as determined by phosphoamino analysis of the $\mathrm{BCR}$ protein, but further information regarding the precise mode of $\mathrm{SH} 2$ domain interaction were not reported. Other work demonstrated that the Abelson murine leukemia virus encoded protein, v-Abl, binds via its $\mathrm{SH} 2$ domain to the adaptor protein Shc with phosphotyrosine-independence. The interaction occurs within the first 85 amino acids of the Shc N-terminus, however the exact binding site was not identified [172].

Another important family of non-receptor tyrosine kinases is the Src gene family, which consists of nine members. Src family kinases are all similarly structured as described earlier. Src family proteins regulate important cellular functions such as growth, adhesion, invasion, and motility [31]. Some of the Src family members are ubiquitously expressed, while others are only present in cells of hematopoietic lineage. Src family members that have been implicated in non-conventional $\mathrm{SH} 2$ domain interactions to date are Blk, Lck, Fyn and c-Src.

Blk is found in $\mathrm{B}$ lymphocytes where the $\mathrm{SH} 2$ domain binds the protein $\mathrm{p} 130^{\mathrm{PITSLRE}}, \mathrm{a}$ high molecular weight homologue of cyclin-dependent kinases. This interaction occurs in a serine and glutamic acid rich region within $\mathrm{p} 130^{\mathrm{PITSLRE}}$, where no tyrosine phosphorylation is detected. Serine phosphorylation of $\mathrm{p} 130^{\text {PITSLRE }}$ by the 
serine/threonine kinase casein kinase II (CKII) is necessary to facilitate Blk SH2 domain binding, utilizing the phosphotyrosine hydrophobic binding pocket. $[173,174]$. The Blk $\mathrm{SH} 2$ domain also binds an acidic region of the protein $\mathrm{p} 150^{\mathrm{TSP}}$, a component of the RNA polymerase II complex, which is devoid of tyrosine residues. However, it is dependent on phosphorylation of serine and/or threonine residues within this acidic region. This interaction was shown to be mediated by the Blk SH2 domain phosphotyrosine binding region [175].

Another Src family cytoplasmic kinase that is found mainly in cells of lymphoid origin and exhibits phosphotyrosine-independent interactions is $p 56^{\text {lck }}$. A series of papers published from the Shin lab show that Lck binds and is regulated by a previously undescribed 62kDa protein [176-178]. This protein was later identified as a member of a novel class of ubiquitin binding proteins that controls signaling in part by ubitiquinmediated protein degradation and forms a structure known as the sequestosome $[178$, 179]. p62, or sequestosome 1, binds the Lck SH2 domain independent of phosphotyrosine, and in a follow-up study, p62 was cloned and the binding specificities with Lck SH2 domain were further determined, mapping the site of interaction between proline 29 and arginine 50 in the p62 amino terminus [176]. However, no specific residue(s) was indicated as being essential for mediating binding.

The Lck SH2 domain also binds the Human Immunodeficiency Virus (HIV) Type 1 Nef protein. Binding assays with the Lck SH2 domain showed that the $\mathrm{SH} 2$ interaction of Nef was reduced when lysates were pre-treated with phosphatase. Nef contains seven tyrosine residues but when mutated individually to phenyalanine, none of the variants affected Lck SH2 binding. Binding was additionally tested with a Lck SH2 
domain phosphotyrosine binding mutant (R134S-R154Q) and no interaction with Nef was observed, although the specific Nef amino acids responsible for the interaction were never deduced [180].

Fyn is a more ubiquitously expressed Src family member that also binds a ligand subset in a SH2-mediated phosphorylation-independent manner. The Fyn $\mathrm{SH} 2$ domain interacts with the serine/threonine kinase Raf-1 when Raf-1 is exclusively serine phosphorylated [181]. Treatment of Raf-1 with phosphatase ablated of the ability of the Fyn $\mathrm{SH} 2$ domain to bind Raf-1, indicating that serine phosphorylation is necessary for the interaction to occur. Binding assays in the presence of phosphotyrosine or phenyl phosphate reduced the interaction, indicating that the hydrophobic pTyr binding pocket in the Fyn SH2 domain is responsible for binding [182].

Src is a ubiquitously expressed protein that plays many important roles in cell growth, adhesion, and motility. The Src SH2 domain also binds Raf-1 and exhibits the same phosphoserine Raf-1 binding requirements as the Fyn $\mathrm{SH} 2$ domain described above. In neurons, Src phosphorylates the N-methyl-D-aspartate subtype of glutamate receptor (NMDAR) to upregulate its activity. Within the activated receptor complex, the protein PSD-95 acts as a negative regulator of Src-induced NMDAR activation. Src binds PSD-95 through $\mathrm{SH} 2$ domain binding independent of tyrsosine phosphorylation. Deletion analysis of PSD-95 identified amino acids 1-54 as the region responsible for Src SH2 binding. Three tyrosine residues present within this region are not responsible for SH2 domain binding. Further mapping narrowed the binding region between amino acids 43-54. Mutation of the phosphotyrosine binding arginine 175 in the SH2 domain did not impact binding, further confirming phosphotyrosine indpendence [183]. 


\section{$\underline{\text { Discussion }}$}

There are a number of reports describing non-conventional, phosphotyrosineindependent SH2 domain interactions with their cognate ligands. The remarkable aspect of these studies is that these binding methods differ from protein to domain, showing very little mechanistic conservation. An interesting speculation could be that these interactions evolved in a very protein-specific manner to suit the signaling needs of cells as organisms became more complex. SH2 domains have appeared in simple, single-celled organisms such as amoeboa [129], so it is no surprise that evolutionary pressure may have occurred to promote such binding events. However, to our knowledge these pTyr-independent interactions have only been shown using $\mathrm{SH} 2$ domains from mammalian proteins. One scenario that comes to mind that could give arise to such a need is that of cells being in a state of nutrient deprivation. In this state, nutrient intake and thus, ATP production would be low, producing a need for a mechanism of basal-state signaling to allow the most basic cellular processes to occur. ATP scarcity would lead to a lack of traditional pTyr binding. The non-conventional SH2 domain binding would allow for critical protein-protein interactions to occur and maintain signaling integrity, enabling cell survival.

Another possibility is that these types of interactions act as priming events for the activation of signaling pathways and cellular functions. In other words, these events could set into motion the series of interactions that would allow tyrosine phosphorylation that creates the conventional $\mathrm{SH} 2$ domain binding sites. We see this in several instances, where tyrosine phosphorylation of proteins is critical for their function due to 
the creation of docking sites for $\mathrm{SH} 2$ domain containing interaction partners. The question is then how do these proteins get phosphorylated in the first place? Of course, kinases that contain $\mathrm{SH} 3$ domains can interact with targets independent of pTyr status; however this would not explain all of the initial phosphorylation events of proteins. Nonconventional binding would allow critical initial phosphorylation events; enabling a litany of translocation, transcription and protein bridging events to occur, giving a "jump start" to initiating cellular signaling systems.

Given the knowledge at hand further studies seeking out and classifying additional phospho-tyrosine independent interactions is warranted. Such studies would lead to a much more in-depth understanding of the intricacies of many different signaling pathways, potentially providing novel targets for therapeutics for a variety of disease states. It will be exciting and enlightening to see what additional new discoveries lay in wait with $\mathrm{SH} 2$ domains as well as other well known interaction domains that are thought to have been exhaustively characterized in terms of function. 


\section{$\underline{\text { References }}$}

1. Rous, P., A Sarcoma of the Fowl Transmissible by an Agent Separable from the Tumor Cells. J Exp Med, 1911. 13(4): p. 397-411.

2. Martin, G.S., The hunting of the Src. Nat Rev Mol Cell Biol, 2001. 2(6): p. 467-75.

3. Sadowski, I., J.C. Stone, and T. Pawson, A noncatalytic domain conserved among cytoplasmic protein-tyrosine kinases modifies the kinase function and transforming activity of Fujinami sarcoma virus P130gag-fps. Mol Cell Biol, 1986. 6(12): p. 4396-408.

4. Mayer, B.J., M. Hamaguchi, and H. Hanafusa, A novel viral oncogene with structural similarity to phospholipase C. Nature, 1988. 332(6161): p. 272-5.

5. $\quad \mathrm{Xu}, \mathrm{W} ., \mathrm{S} . \mathrm{C}$. Harrison, and M.J. Eck, Three-dimensional structure of the tyrosine kinase c-Src. Nature, 1997. 385(6617): p. 595-602.

6. $\mathrm{Xu}, \mathrm{W}$. , et al., Crystal structures of c-Src reveal features of its autoinhibitory mechanism. Mol Cell, 1999. 3(5): p. 629-38.

7. Young, M.A., et al., Dynamic coupling between the SH2 and SH3 domains of c-Src and Hck underlies their inactivation by C-terminal tyrosine phosphorylation. Cell, 2001. 105(1): p. 115-26.

8. Bjorge, J.D., A. Pang, and D.J. Fujita, Identification of protein-tyrosine phosphatase $1 B$ as the major tyrosine phosphatase activity capable of dephosphorylating and activating c-Src in several human breast cancer cell lines. J Biol Chem, 2000. 275(52): p. 41439-46.

9. Parsons, J.T. and M.J. Weber, Genetics of src: structure and functional organization of a protein tyrosine kinase. Curr Top Microbiol Immunol, 1989. 147: p. 79-127.

10. Giannoni, E., et al., Intracellular reactive oxygen species activate Src tyrosine kinase during cell adhesion and anchorage-dependent cell growth. Mol Cell Biol, 2005. 25(15): p. 6391-403.

11. Schaller, M.D., et al., Autophosphorylation of the focal adhesion kinase, pp125FAK, directs SH2dependent binding of pp60src. Mol Cell Biol, 1994. 14(3): p. 1680-8.

12. Burnham, M.R., et al., Regulation of c-SRC activity and function by the adapter protein CAS. Mol Cell Biol, 2000. 20(16): p. 5865-78.

13. Thomas, J.W., et al., SH2- and SH3-mediated interactions between focal adhesion kinase and Src. J Biol Chem, 1998. 273(1): p. 577-83.

14. Tice, D.A., et al., Mechanism of biological synergy between cellular Src and epidermal growth factor receptor. Proc Natl Acad Sci U S A, 1999. 96(4): p. 1415-20.

15. Cooper, J.A., et al., Tyr527 is phosphorylated in pp60c-src: implications for regulation. Science, 1986. 231(4744): p. 1431-4.

16. Kim, M., et al., Cbl-c suppresses v-Src-induced transformation through ubiquitin-dependent protein degradation. Oncogene, 2004. 23(9): p. 1645-55.

17. Frame, M.C., Newest findings on the oldest oncogene; how activated src does it. J Cell Sci, 2004. 117(Pt 7): p. 989-98.

18. Reynolds, A.B., et al., Activation of the oncogenic potential of the avian cellular src protein by specific structural alteration of the carboxy terminus. EMBO J, 1987. 6(8): p. 2359-64.

19. Catling, A.D., et al., Mutations in v-SrC SH3 and catalytic domains that jointly confer temperature-sensitive transformation with minimal temperature-dependent changes in cellular tyrosine phosphorylation. J Virol, 1994. 68(7): p. 4392-9.

20. Tian, M. and G.S. Martin, Reduced phosphotyrosine binding by the v-Src SH2 domain is compatible with wild-type transformation. Oncogene, 1996. 12(4): p. 727-34.

21. Weng, Z., et al., Structure-function analysis of SH3 domains: SH3 binding specificity altered by single amino acid substitutions. Mol Cell Biol, 1995. 15(10): p. 5627-34. 
22. Snyder, M.A., et al., A mutation at the ATP-binding site of pp60v-src abolishes kinase activity, transformation, and tumorigenicity. Mol Cell Biol, 1985. 5(7): p. 1772-9.

23. Mori, S., et al., Identification of two juxtamembrane autophosphorylation sites in the PDGF betareceptor; involvement in the interaction with Src family tyrosine kinases. EMBO J, 1993. 12(6): p. 2257-64.

24. Frame, M.C., Src in cancer: deregulation and consequences for cell behaviour. Biochim Biophys Acta, 2002. 1602(2): p. 114-30.

25. Riley, D., et al., The mechanism of cell cycle regulation by v-Src. Oncogene, 2001. 20(42): p. 5941-50.

26. Bromberg, J.F., et al., Epidermal growth factor-induced growth inhibition requires Stat1 activation. Cell Growth Differ, 1998. 9(7): p. 505-12.

27. Turkson, J., et al., Requirement for Ras/Rac1-mediated $p 38$ and c-Jun N-terminal kinase signaling in Stat3 transcriptional activity induced by the Src oncoprotein. Mol Cell Biol, 1999. 19(11): p. 7519-28.

28. Roche, S., et al., Requirement of phospholipase C gamma, the tyrosine phosphatase Syp and the adaptor proteins Shc and Nck for PDGF-induced DNA synthesis: evidence for the existence of Rasdependent and Ras-independent pathways. EMBO J, 1996. 15(18): p. 4940-8.

29. Olayioye, M.A., et al., ErbB receptor-induced activation of stat transcription factors is mediated by Src tyrosine kinases. J Biol Chem, 1999. 274(24): p. 17209-18.

30. Sastry, S.K. and K. Burridge, Focal adhesions: a nexus for intracellular signaling and cytoskeletal dynamics. Exp Cell Res, 2000. 261(1): p. 25-36.

31. Yeatman, T.J., A renaissance for SRC. Nat Rev Cancer, 2004. 4(6): p. 470-80.

32. Felsenfeld, D.P., et al., Selective regulation of integrin--cytoskeleton interactions by the tyrosine kinase Src. Nat Cell Biol, 1999. 1(4): p. 200-6.

33. Frame, M.C., et al., v-Src's hold over actin and cell adhesions. Nat Rev Mol Cell Biol, 2002. 3(4): p. 233-45.

34. Fujita, Y., et al., Hakai, a c-Cbl-like protein, ubiquitinates and induces endocytosis of the Ecadherin complex. Nat Cell Biol, 2002. 4(3): p. 222-31.

35. Hynes, R.O., Integrins: versatility, modulation, and signaling in cell adhesion. Cell, 1992. 69(1): p. 11-25.

36. Glading, A., et al., Membrane proximal ERK signaling is required for M-calpain activation downstream of epidermal growth factor receptor signaling. J Biol Chem, 2001. 276(26): p. 23341-8.

37. Sieg, D.J., et al., FAK integrates growth-factor and integrin signals to promote cell migration. Nat Cell Biol, 2000. 2(5): p. 249-56.

38. Lauffenburger, D.A. and A.F. Horwitz, Cell migration: a physically integrated molecular process. Cell, 1996. 84(3): p. 359-69.

39. Laukaitis, C.M., et al., Differential dynamics of alpha 5 integrin, paxillin, and alpha-actinin during formation and disassembly of adhesions in migrating cells. J Cell Biol, 2001. 153(7): p. 1427-40.

40. Burridge, K. and M. Chrzanowska-Wodnicka, Focal adhesions, contractility, and signaling. Annu Rev Cell Dev Biol, 1996. 12: p. 463-518.

41. Ammer, A.G. and S.A. Weed, Cortactin branches out: roles in regulating protrusive actin dynamics. Cell Motil Cytoskeleton, 2008. 65(9): p. 687-707.

42. Schaller, M.D. and J.T. Parsons, pp125FAK-dependent tyrosine phosphorylation of paxillin creates a high-affinity binding site for Crk. Mol Cell Biol, 1995. 15(5): p. 2635-45.

43. Playford, M.P. and M.D. Schaller, The interplay between Src and integrins in normal and tumor biology. Oncogene, 2004. 23(48): p. 7928-46. 
44. Fincham, V.J., A. Chudleigh, and M.C. Frame, Regulation of 190 Rho-GAP by v-Src is linked to cytoskeletal disruption during transformation. J Cell Sci, 1999. 112 ( Pt 6): p. 947-56.

45. Arthur, W.T. and K. Burridge, RhoA inactivation by 190 RhoGAP regulates cell spreading and migration by promoting membrane protrusion and polarity. Mol Biol Cell, 2001. 12(9): p. 271120.

46. Nobes, C.D. and A. Hall, Rho, rac, and cdc42 GTPases regulate the assembly of multimolecular focal complexes associated with actin stress fibers, lamellipodia, and filopodia. Cell, 1995. 81(1): p. 53-62.

47. Nobes, C.D. and A. Hall, Rho GTPases control polarity, protrusion, and adhesion during cell movement. J Cell Biol, 1999. 144(6): p. 1235-44.

48. Wu, H., et al., Identification and characterization of a novel cytoskeleton-associated pp60src substrate. Mol Cell Biol, 1991. 11(10): p. 5113-24.

49. Weaver, A.M., et al., Integration of signals to the Arp2/3 complex. Curr Opin Cell Biol, 2003. 15(1): p. 23-30.

50. Weaver, A.M., et al., Interaction of cortactin and N-WASp with Arp2/3 complex. Curr Biol, 2002. 12(15): p. 1270-8.

51. Pollard, T.D., Regulation of actin filament assembly by Arp2/3 complex and formins. Annu Rev Biophys Biomol Struct, 2007. 36: p. 451-77.

52. Miglarese, M.R., et al., The protein tyrosine kinase substrate cortactin is differentially expressed in murine B lymphoid tumors. Oncogene, 1994. 9(7): p. 1989-97.

53. Du, Y., et al., Identification of a novel cortactin SH3 domain-binding protein and its localization to growth cones of cultured neurons. Mol Cell Biol, 1998. 18(10): p. 5838-51.

54. Weed, S.A., Y. Du, and J.T. Parsons, Translocation of cortactin to the cell periphery is mediated by the small GTPase Rac1. J Cell Sci, 1998. 111 ( Pt 16): p. 2433-43.

55. Daly, R.J., Cortactin signalling and dynamic actin networks. Biochem J, 2004. 382(Pt 1): p. 13-25.

56. Weed, S.A., et al., Cortactin localization to sites of actin assembly in lamellipodia requires interactions with F-actin and the Arp2/3 complex. J Cell Biol, 2000. 151(1): p. 29-40.

57. Galletta, B.J., D.Y. Chuang, and J.A. Cooper, Distinct roles for Arp2/3 regulators in actin assembly and endocytosis. PLoS Biol, 2008. 6(1): p. e1.

58. Zhang, X., et al., HDAC6 modulates cell motility by altering the acetylation level of cortactin. Mol Cell, 2007. 27(2): p. 197-213.

59. Webb, B.A., et al., Phosphorylation of cortactin by p21-activated kinase. Arch Biochem Biophys, 2006. 456(2): p. 183-93.

60. Huang, C., et al., Proteolysis of platelet cortactin by calpain. J Biol Chem, 1997. 272(31): p. 19248-52.

61. Perrin, B.J., K.J. Amann, and A. Huttenlocher, Proteolysis of cortactin by calpain regulates membrane protrusion during cell migration. Mol Biol Cell, 2006. 17(1): p. 239-50.

62. Campbell, D.H., R.L. Sutherland, and R.J. Daly, Signaling pathways and structural domains required for phosphorylation of EMS1/cortactin. Cancer Res, 1999. 59(20): p. 5376-85.

63. Evans, J.V., et al., Src binds cortactin through a SH2 domain cystine-mediated linkage. J Cell Sci, 2012.

64. Lapetina, S., et al., Arg interacts with cortactin to promote adhesion-dependent cell edge protrusion. J Cell Biol, 2009. 185(3): p. 503-19.

65. Oser, M., et al., Cortactin regulates cofilin and N-WASp activities to control the stages of invadopodium assembly and maturation. J Cell Biol, 2009. 186(4): p. 571-87.

66. McNiven, M.A., et al., Regulated interactions between dynamin and the actin-binding protein cortactin modulate cell shape. J Cell Biol, 2000. 151(1): p. 187-98. 
67. Tomar, A., et al., Cortactin as a target for FAK in the regulation of focal adhesion dynamics. PLoS One, 2012. 7(8): p. e44041.

68. Ren, G., M.S. Crampton, and A.S. Yap, Cortactin: Coordinating adhesion and the actin cytoskeleton at cellular protrusions. Cell Motil Cytoskeleton, 2009. 66(10): p. 865-73.

69. Liotta, L.A., et al., Tumor cell motility. Semin Cancer Biol, 1991. 2(2): p. 111-4.

70. Ponti, A., et al., Two distinct actin networks drive the protrusion of migrating cells. Science, 2004. 305(5691): p. 1782-6.

71. Orth, J.D. and M.A. McNiven, Get off my back! Rapid receptor internalization through circular dorsal ruffles. Cancer Res, 2006. 66(23): p. 11094-6.

72. Yamaguchi, H. and J. Condeelis, Regulation of the actin cytoskeleton in cancer cell migration and invasion. Biochim Biophys Acta, 2007. 1773(5): p. 642-52.

73. Urrutia, R., et al., The dynamins: redundant or distinct functions for an expanding family of related GTPases? Proc Natl Acad Sci U S A, 1997. 94(2): p. 377-84.

74. Schafer, D.A., et al., Dynamin2 and cortactin regulate actin assembly and filament organization. Curr Biol, 2002. 12(21): p. 1852-7.

75. Cao, H., et al., Cortactin is a component of clathrin-coated pits and participates in receptormediated endocytosis. Mol Cell Biol, 2003. 23(6): p. 2162-70.

76. Kaksonen, M., H.B. Peng, and H. Rauvala, Association of cortactin with dynamic actin in lamellipodia and on endosomal vesicles. J Cell Sci, 2000. 113 Pt 24: p. 4421-6.

77. Pant, K., et al., Cortactin binding to F-actin revealed by electron microscopy and $3 \mathrm{D}$ reconstruction. J Mol Biol, 2006. 359(4): p. 840-7.

78. McLaughlin, P.J., et al., Structure of gelsolin segment 1-actin complex and the mechanism of filament severing. Nature, 1993. 364(6439): p. 685-92.

79. Dominguez, R., Actin-binding proteins--a unifying hypothesis. Trends Biochem Sci, 2004. 29(11): p. 572-8.

80. Bryce, N.S., et al., Cortactin promotes cell motility by enhancing lamellipodial persistence. Curr Biol, 2005. 15(14): p. 1276-85.

81. Weaver, A.M., et al., Cortactin promotes and stabilizes Arp2/3-induced actin filament network formation. Curr Biol, 2001. 11(5): p. 370-4.

82. Blanchoin, L., T.D. Pollard, and R.D. Mullins, Interactions of ADF/cofilin, Arp2/3 complex, capping protein and profilin in remodeling of branched actin filament networks. Curr Biol, 2000. 10(20): p. 1273-82.

83. Rinnerthaler, G., B. Geiger, and J.V. Small, Contact formation during fibroblast locomotion: involvement of membrane ruffles and microtubules. J Cell Biol, 1988. 106(3): p. 747-60.

84. Small, J.V., M. Herzog, and K. Anderson, Actin filament organization in the fish keratocyte lamellipodium. J Cell Biol, 1995. 129(5): p. 1275-86.

85. Machesky, L.M., et al., Scar, a WASp-related protein, activates nucleation of actin filaments by the Arp2/3 complex. Proc Natl Acad Sci U S A, 1999. 96(7): p. 3739-44.

86. Uruno, T., et al., Sequential interaction of actin-related proteins 2 and 3 (Arp2/3) complex with neural Wiscott-Aldrich syndrome protein (N-WASP) and cortactin during branched actin filament network formation. J Biol Chem, 2003. 278(28): p. 26086-93.

87. Mizutani, K., et al., Essential role of neural Wiskott-Aldrich syndrome protein in podosome formation and degradation of extracellular matrix in src-transformed fibroblasts. Cancer Res, 2002. 62(3): p. 669-74.

88. Kinley, A.W., et al., Cortactin interacts with WIP in regulating Arp2/3 activation and membrane protrusion. Curr Biol, 2003. 13(5): p. 384-93.

89. Tehrani, S., et al., Src phosphorylation of cortactin enhances actin assembly. Proc Natl Acad Sci U S A, 2007. 104(29): p. 11933-8. 
90. Reynolds, A.B., et al., Transformation-specific tyrosine phosphorylation of a novel cellular protein in chicken cells expressing oncogenic variants of the avian cellular src gene. Mol Cell Biol, 1989. 9(2): p. 629-38.

91. Wu, H. and J.T. Parsons, Cortactin, an 80/85-kilodalton pp60src substrate, is a filamentous actinbinding protein enriched in the cell cortex. J Cell Biol, 1993. 120(6): p. 1417-26.

92. Zhan, X., et al., Association of fibroblast growth factor receptor-1 with c-Src correlates with association between c-Src and cortactin. J Biol Chem, 1994. 269(32): p. 20221-4.

93. LaVallee, T.M., et al., Activation of the MAP kinase pathway by FGF-1 correlates with cell proliferation induction while activation of the Src pathway correlates with migration. J Cell Biol, 1998. 141(7): p. 1647-58.

94. Liu, J., C. Huang, and X. Zhan, Src is required for cell migration and shape changes induced by fibroblast growth factor 1. Oncogene, 1999. 18(48): p. 6700-6.

95. Okamura, H. and M.D. Resh, p80/85 cortactin associates with the Src SH2 domain and colocalizes with $v$-Src in transformed cells. J Biol Chem, 1995. 270(44): p. 26613-8.

96. Vuori, K. and E. Ruoslahti, Tyrosine phosphorylation of p130Cas and cortactin accompanies integrin-mediated cell adhesion to extracellular matrix. J Biol Chem, 1995. 270(38): p. 22259-62.

97. Huang, C., et al., Down-regulation of the filamentous actin cross-linking activity of cortactin by Src-mediated tyrosine phosphorylation. J Biol Chem, 1997. 272(21): p. 13911-5.

98. Li, Y., J. Liu, and X. Zhan, Tyrosine phosphorylation of cortactin is required for H2O2-mediated injury of human endothelial cells. J Biol Chem, 2000. 275(47): p. 37187-93.

99. Li, Y., et al., Cortactin potentiates bone metastasis of breast cancer cells. Cancer Res, 2001. 61(18): p. 6906-11.

100. Patel, A.S., et al., Overexpression of EMS1/cortactin in NIH3T3 fibroblasts causes increased cell motility and invasion in vitro. Oncogene, 1998. 16(25): p. 3227-32.

101. Martinez-Quiles, N., et al., Erk/Src phosphorylation of cortactin acts as a switch on-switch off mechanism that controls its ability to activate N-WASP. Mol Cell Biol, 2004. 24(12): p. 5269-80.

102. Head, J.A., et al., Cortactin tyrosine phosphorylation requires Rac1 activity and association with the cortical actin cytoskeleton. Mol Biol Cell, 2003. 14(8): p. 3216-29.

103. Oser, M., et al., Specific tyrosine phosphorylation sites on cortactin regulate Nck1-dependent actin polymerization in invadopodia. J Cell Sci, 2010. 123(Pt 21): p. 3662-73.

104. Kelley, L.C., et al., Cortactin phosphorylated by ERK1/2 localizes to sites of dynamic actin regulation and is required for carcinoma lamellipodia persistence. PLoS One, 2011. 5(11): p. e13847.

105. Kelley, L.C., et al., Revisiting the ERK/Src cortactin switch. Commun Integr Biol. 4(2): p. 205-7.

106. Weaver, A.M., Cortactin in tumor invasiveness. Cancer Lett, 2008. 265(2): p. 157-66.

107. Ammer, A.G., et al., Saracatinib Impairs Head and Neck Squamous Cell Carcinoma Invasion by Disrupting Invadopodia Function. J Cancer Sci Ther, 2009. 1(2): p. 52-61.

108. Matsubara, T., et al., Critical role of cortactin in actin ring formation and osteoclastic bone resorption. J Bone Miner Metab, 2006. 24(5): p. 368-72.

109. Tehrani, S., et al., Cortactin has an essential and specific role in osteoclast actin assembly. Mol Biol Cell, 2006. 17(7): p. 2882-95.

110. Zhou, S., et al., Effects of tyrosine phosphorylation of cortactin on podosome formation in A7r5 vascular smooth muscle cells. Am J Physiol Cell Physiol, 2006. 290(2): p. C463-71.

111. Bowden, E.T., et al., Co-localization of cortactin and phosphotyrosine identifies active invadopodia in human breast cancer cells. Exp Cell Res, 2006. 312(8): p. 1240-53.

112. Webb, B.A., et al., Dissecting the functional domain requirements of cortactin in invadopodia formation. Eur J Cell Biol, 2007. 86(4): p. 189-206. 
113. Artym, V.V., et al., Dynamic interactions of cortactin and membrane type 1 matrix metalloproteinase at invadopodia: defining the stages of invadopodia formation and function. Cancer Res, 2006. 66(6): p. 3034-43.

114. Kelley, L.C., et al., Oncogenic Src requires a wild-type counterpart to regulate invadopodia maturation. J Cell Sci, 2010. 123(Pt 22): p. 3923-32.

115. Koppikar, P., et al., Combined inhibition of c-Src and epidermal growth factor receptor abrogates growth and invasion of head and neck squamous cell carcinoma. Clin Cancer Res, 2008. 14(13): p. 4284-91.

116. Magalhaes, M.A., et al., Cortactin phosphorylation regulates cell invasion through a pHdependent pathway. J Cell Biol, 2011. 195(5): p. 903-20.

117. Dehio, C., M.C. Prevost, and P.J. Sansonetti, Invasion of epithelial cells by Shigella flexneri induces tyrosine phosphorylation of cortactin by a pp60c-src-mediated signalling pathway. EMBO J, 1995. 14(11): p. 2471-82.

118. Dumenil, G., et al., Interferon alpha inhibits a Src-mediated pathway necessary for Shigellainduced cytoskeletal rearrangements in epithelial cells. J Cell Biol, 1998. 143(4): p. 1003-12.

119. Hoffmann, I., et al., Activation of ErbB2 receptor tyrosine kinase supports invasion of endothelial cells by Neisseria meningitidis. J Cell Biol, 2001. 155(1): p. 133-43.

120. Selbach, M., et al., The Helicobacter pylori CagA protein induces cortactin dephosphorylation and actin rearrangement by c-Src inactivation. EMBO J, 2003. 22(3): p. 515-28.

121. Kinnunen, T., et al., Cortactin-Src kinase signaling pathway is involved in N-syndecan-dependent neurite outgrowth. J Biol Chem, 1998. 273(17): p. 10702-8.

122. Yang, L., et al., Endothelial cell cortactin phosphorylation by Src contributes to polymorphonuclear leukocyte transmigration in vitro. Circ Res, 2006. 98(3): p. 394-402.

123. Zhu, J., et al., Receptor-mediated endocytosis involves tyrosine phosphorylation of cortactin. J Biol Chem, 2007. 282(22): p. 16086-94.

124. Singh, V.P. and M.A. McNiven, Src-mediated cortactin phosphorylation regulates actin localization and injurious blebbing in acinar cells. Mol Biol Cell, 2008. 19(5): p. 2339-47.

125. Kapus, A., et al., Cell shrinkage regulates Src kinases and induces tyrosine phosphorylation of cortactin, independent of the osmotic regulation of $\mathrm{Na}+\mathrm{H}+$ exchangers. J Biol Chem, 1999. 274(12): p. 8093-102.

126. Zahradka, P., B. Storie, and B. Wright, IGF-1 receptor transactivation mediates Src-dependent cortactin phosphorylation in response to angiotensin II. Can J Physiol Pharmacol, 2009. 87(10): p. 805-12.

127. Campbell, S.J. and R.M. Jackson, Diversity in the SH2 domain family phosphotyrosyl peptide binding site. Protein Eng, 2003. 16(3): p. 217-27.

128. Margolis, B., Proteins with SH2 domains: transducers in the tyrosine kinase signaling pathway. Cell Growth Differ, 1992. 3(1): p. 73-80.

129. Liu, B.A., et al., The SH2 domain-containing proteins in 21 species establish the provenance and scope of phosphotyrosine signaling in eukaryotes. Sci Signal, 2010. 4(202): p. ra83.

130. Koch, C.A., et al., SH2 and SH3 domains: elements that control interactions of cytoplasmic signaling proteins. Science, 1991. 252(5006): p. 668-74.

131. Filippakopoulos, P., S. Muller, and S. Knapp, SH2 domains: modulators of nonreceptor tyrosine kinase activity. Curr Opin Struct Biol, 2009. 19(6): p. 643-9.

132. Rameh, L.E., C.S. Chen, and L.C. Cantley, Phosphatidylinositol $(3,4,5) P 3$ interacts with SH2 domains and modulates PI 3-kinase association with tyrosine-phosphorylated proteins. Cell, 1995. 83(5): p. 821-30.

133. Bae, J.H., et al., The selectivity of receptor tyrosine kinase signaling is controlled by a secondary SH2 domain binding site. Cell, 2009. 138(3): p. 514-24. 
134. Sayos, J., et al., The X-linked lymphoproliferative-disease gene product SAP regulates signals induced through the co-receptor SLAM. Nature, 1998. 395(6701): p. 462-9.

135. Poy, F., et al., Crystal structures of the XLP protein SAP reveal a class of SH2 domains with extended, phosphotyrosine-independent sequence recognition. Mol Cell, 1999. 4(4): p. 555-61.

136. Li, S.C., et al., Novel mode of ligand binding by the SH2 domain of the human XLP disease gene product SAP/SH2D1A. Curr Biol, 1999. 9(23): p. 1355-62.

137. Morra, M., et al., Structural basis for the interaction of the free SH2 domain EAT-2 with SLAM receptors in hematopoietic cells. EMBO J, 2001. 20(21): p. 5840-52.

138. Dechert, U., et al., Characterization of protein tyrosine phosphatase SH-PTP2. Study of phosphopeptide substrates and possible regulatory role of SH2 domains. J Biol Chem, 1994. 269(8): p. 5602-11.

139. Joseph, R.E., et al., A remote substrate docking mechanism for the tec family tyrosine kinases. Biochemistry, 2007. 46(18): p. 5595-603.

140. Filippakopoulos, P., et al., Structural coupling of SH2-kinase domains links Fes and Abl substrate recognition and kinase activation. Cell, 2008. 134(5): p. 793-803.

141. Choi, J.H., S.H. Ryu, and P.G. Suh, On/off-regulation of phospholipase C-gamma 1-mediated signal transduction. Adv Enzyme Regul, 2007. 47: p. 104-16.

142. Rhee, S.G., Regulation of phosphoinositide-specific phospholipase C. Annu Rev Biochem, 2001. 70: p. 281-312.

143. Middlemas, D.S., J. Meisenhelder, and T. Hunter, Identification of TrkB autophosphorylation sites and evidence that phospholipase C-gamma 1 is a substrate of the TrkB receptor. J Biol Chem, 1994. 269(7): p. 5458-66.

144. Kim, J.W., et al., Tyrosine residues in bovine phospholipase C-gamma phosphorylated by the epidermal growth factor receptor in vitro. J Biol Chem, 1990. 265(7): p. 3940-3.

145. Alava, M.A., et al., Increased intracellular cyclic AMP inhibits inositol phospholipid hydrolysis induced by perturbation of the T cell receptor/CD3 complex but not by G-protein stimulation. Association with protein kinase A-mediated phosphorylation of phospholipase C-gamma 1. Biochem J, 1992. 284 ( Pt 1): p. 189-99.

146. Bae, S.S., et al., Regulation of phospholipase C-gamma1 by protein kinase A-dependent phosphorylation. Adv Enzyme Regul, 2002. 42: p. 195-211.

147. Nanney, L.B., et al., Altered distribution of phospholipase C-gamma 1 in benign hyperproliferative epidermal diseases. Cell Growth Differ, 1992. 3(4): p. 233-9.

148. Muller, A.J., et al., A limited set of SH2 domains binds BCR through a high-affinity phosphotyrosine-independent interaction. Mol Cell Biol, 1992. 12(11): p. 5087-93.

149. Vigil, D., et al., Ras superfamily GEFs and GAPs: validated and tractable targets for cancer therapy? Nat Rev Cancer, 2010. 10(12): p. 842-57.

150. Pertz, O., Spatio-temporal Rho GTPase signaling - where are we now? J Cell Sci, 2010. 123(Pt 11): p. 1841-50.

151. Mahajan, N.P. and H.S. Earp, An SH2 domain-dependent, phosphotyrosine-independent interaction between Vav1 and the Mer receptor tyrosine kinase: a mechanism for localizing guanine nucleotide-exchange factor action. J Biol Chem, 2003. 278(43): p. 42596-603.

152. Han, D.C., T.L. Shen, and J.L. Guan, The Grb7 family proteins: structure, interactions with other signaling molecules and potential cellular functions. Oncogene, 2001. 20(44): p. 6315-21.

153. Morrione, A., Grb10 adapter protein as regulator of insulin-like growth factor receptor signaling. J Cell Physiol, 2003. 197(3): p. 307-11.

154. Oligino, L., et al., Nonphosphorylated peptide ligands for the Grb2 Src homology 2 domain. J Biol Chem, 1997. 272(46): p. 29046-52. 
155. Pero, S.C., et al., Identification of novel non-phosphorylated ligands, which bind selectively to the SH2 domain of Grb7. J Biol Chem, 2002. 277(14): p. 11918-26.

156. Nantel, A., et al., Interaction of the Grb10 adapter protein with the Raf1 and MEK1 kinases. J Biol Chem, 1998. 273(17): p. 10475-84.

157. Morrione, A., et al., mGrb10 interacts with Nedd4. J Biol Chem, 1999. 274(34): p. 24094-9.

158. Huang, Q. and D.M. Szebenyi, Structural basis for the interaction between the growth factorbinding protein GRB10 and the E3 ubiquitin ligase NEDD4. J Biol Chem, 2010. 285(53): p. 421309.

159. Qian, X., et al., The Tensin-3 protein, including its SH2 domain, is phosphorylated by Src and contributes to tumorigenesis and metastasis. Cancer Cell, 2009. 16(3): p. 246-58.

160. Albasri, A., et al., CTEN (C-terminal tensin-like), a novel oncogene overexpressed in invasive breast carcinoma of poor prognosis. Breast Cancer Res Treat, 2011. 126(1): p. 47-54.

161. Liao, Y.C., et al., The phosphotyrosine-independent interaction of DLC-1 and the SH2 domain of cten regulates focal adhesion localization and growth suppression activity of DLC-1. J Cell Biol, 2007. 176(1): p. 43-9.

162. Dai, K., et al., Solution structure of tensin2 SH2 domain and its phosphotyrosine-independent interaction with DLC-1. PLoS One, 2011. 6(7): p. e21965.

163. De Sepulveda, P., S. Ilangumaran, and R. Rottapel, Suppressor of cytokine signaling-1 inhibits VAV function through protein degradation. J Biol Chem, 2000. 275(19): p. 14005-8.

164. Schmandt, R., S.K. Liu, and C.J. McGlade, Cloning and characterization of MPAL, a novel Shc SH2 domain-binding protein expressed in proliferating cells. Oncogene, 1999. 18(10): p. 1867-79.

165. Johnston, P.A. and J.R. Grandis, STAT3 signaling: anticancer strategies and challenges. Mol Interv, 2011. 11(1): p. 18-26.

166. Ram, P.T. and R. lyengar, $G$ protein coupled receptor signaling through the Src and Stat3 pathway: role in proliferation and transformation. Oncogene, 2001. 20(13): p. 1601-6.

167. Zhao, L. and P.K. Vogt, Class I PI3K in oncogenic cellular transformation. Oncogene, 2008. 27(41): p. 5486-96.

168. Liu, P., et al., Targeting the phosphoinositide 3-kinase pathway in cancer. Nat Rev Drug Discov, 2009. 8(8): p. 627-44.

169. King, T.R., et al., Using a phage display library to identify basic residues in A-Raf required to mediate binding to the Src homology 2 domains of the p85 subunit of phosphatidylinositol 3'kinase. J Biol Chem, 2000. 275(46): p. 36450-6.

170. Fang, Y., et al., Two phosphorylation-independent sites on the p85 SH2 domains bind A-Raf kinase. Biochem Biophys Res Commun, 2002. 290(4): p. 1267-74.

171. Colicelli, J., $A B L$ tyrosine kinases: evolution of function, regulation, and specificity. Sci Signal, 2010. 3(139): p. re6.

172. Raffel, G.D., K. Parmar, and N. Rosenberg, In vivo association of v-Ab/ with Shc mediated by a non-phosphotyrosine-dependent SH2 interaction. J Biol Chem, 1996. 271(9): p. 4640-5.

173. Malek, S.N. and S. Desiderio, SH2 domains of the protein-tyrosine kinases Blk, Lyn, and Fyn(T) bind distinct sets of phosphoproteins from B lymphocytes. J Biol Chem, 1993. 268(30): p. 2255765.

174. Malek, S.N. and S. Desiderio, A cyclin-dependent kinase homologue, p13OPITSLRE is a phosphotyrosine-independent SH2 ligand. J Biol Chem, 1994. 269(52): p. 33009-20.

175. Malek, S.N., et al., p150TSP, a conserved nuclear phosphoprotein that contains multiple tetratricopeptide repeats and binds specifically to SH2 domains. J Biol Chem, 1996. 271(12): p. 6952-62.

176. Joung, I., J.L. Strominger, and J. Shin, Molecular cloning of a phosphotyrosine-independent ligand of the p56/ck SH2 domain. Proc Natl Acad Sci U S A, 1996. 93(12): p. 5991-5. 
177. Park, I., et al., Phosphotyrosine-independent binding of a 62-kDa protein to the src homology 2 (SH2) domain of p56lck and its regulation by phosphorylation of Ser-59 in the lck unique Nterminal region. Proc Natl Acad Sci U S A, 1995. 92(26): p. 12338-42.

178. Vadlamudi, R.K., et al., p62, a phosphotyrosine-independent ligand of the SH2 domain of p56/ck, belongs to a new class of ubiquitin-binding proteins. J Biol Chem, 1996. 271(34): p. 20235-7.

179. Shin, J., P62 and the sequestosome, a novel mechanism for protein metabolism. Arch Pharm Res, 1998. 21(6): p. 629-33.

180. Dutartre, H., et al., The human immunodeficiency virus type 1 Nef protein binds the Src-related tyrosine kinase Lck SH2 domain through a novel phosphotyrosine independent mechanism. Virology, 1998. 247(2): p. 200-11.

181. Morrison, D.K., et al., Direct activation of the serine/threonine kinase activity of Raf-1 through tyrosine phosphorylation by the PDGF beta-receptor. Cell, 1989. 58(4): p. 649-57.

182. Cleghon, V. and D.K. Morrison, Raf-1 interacts with Fyn and Src in a non-phosphotyrosinedependent manner. J Biol Chem, 1994. 269(26): p. 17749-55.

183. Kalia, L.V., et al., PSD-95 is a negative regulator of the tyrosine kinase Src in the NMDA receptor complex. EMBO J, 2006. 25(20): p. 4971-82. 


\title{
Study 1: Src Binds Cortactin Through an SH2 Domain Cystine-mediated Linkage
}

\author{
Jason V. Evans ${ }^{1}$, Amanda G. Ammer ${ }^{1 \dagger}$, John E. Jett ${ }^{2 \dagger}$, Chris A. Bolcato ${ }^{4}$, Jason C. \\ Breaux $^{4}$, Karen H. Martin ${ }^{1}$, Mark V. Culp ${ }^{3}$, Peter M. Gannett ${ }^{2}$, and Scott A. Weed ${ }^{1^{*}}$ \\ ${ }^{1}$ Departments of Neurobiology and Anatomy, Program in Cancer Cell Biology, Mary \\ Babb Randolph Cancer Center \\ ${ }^{2}$ Department of Pharmaceutical and Pharmacological Sciences \\ ${ }^{3}$ Department of Statistics \\ West Virginia University, Morgantown, WV 26506, USA \\ ${ }^{4}$ Protea Biosciences, Inc., Morgantown, WV 26507, USA \\ *Correspondence: sweed@hsc.wvu.edu \\ ${ }^{\dagger}$ Equal Contribution
}

Running title: Cystine bonding between Src and cortactin Keywords: Src, SH2 domain, cortactin, cystine 


\section{Summary}

Tyrosine kinase-based signal transduction mediated by modular protein domains is critical for cellular function. The Src homology (SH)2 domain is an important conductor of intracellular signalling that binds to phosphorylated tyrosines on acceptor proteins, producing molecular complexes responsible for signal relay. Cortactin is a cytoskeletal protein and tyrosine kinase substrate that regulates actin-based motility through interactions with $\mathrm{SH} 2$ domain-containing proteins. The $\mathrm{Src}$ kinase $\mathrm{SH} 2$ domain mediates cortactin binding and tyrosine phosphorylation, but how Src interacts with cortactin is unknown. Here we demonstrate that Src binds cortactin through cystine bonding between $\mathrm{Src}$ C185 in the $\mathrm{SH} 2$ domain within the phosphotyrosine binding pocket and cortactin C112/246 in the cortactin repeats domain independent of tyrosine phosphorylation. Interaction studies in the presence of reducing agents ablate Srccortactin binding, eliminates cortactin phosphorylation by Src, and prevents $\mathrm{Src} \mathrm{SH} 2$ domain binding to cortactin. Tandem MS/MS sequencing demonstrates cystine bond formation between Src C185 and cortactin C112/246. Mutational studies indicate that an intact cystine binding interface is required for Src-mediated cortactin phosphorylation, cell migration, and pre-invadopodia formation. Our results identify a novel phosphotyrosine independent binding mode between the $\mathrm{Src} \mathrm{SH} 2$ domain and cortactin. Besides Src, one quarter of all SH2 domains contain cysteines at or near the analogous Src C185 position. This provides a potential alternative mechanism to tyrosine phosphorylation for cysteine-containing $\mathrm{SH} 2$ domains to bind cognate ligands that may be widespread in propagating signals regulating diverse cellular functions. 


\section{Introduction}

Signal transduction through protein-protein interactions is essential for cellular function and is mediated by specialized protein domains. The $\mathrm{SH} 2$ domain is one of the initially discovered and best-characterized protein interaction motifs [1]. SH2 domains are $\sim 100$ amino acids in length, and genomic analysis indicates that $121 \mathrm{SH} 2$ domains are found in 115 individual human proteins that participate in a wide range of signaling events [2]. SH2 domains function by binding to phosphorylated tyrosine residues in target proteins typically specified by residues in positions -2 to +4 of the phosphotyrosine $[3,4]$. SH2 domains are structurally conserved, consisting of a $\beta$-sheet flanked by opposing a-helices [5]. A positively charged binding pocket within the $\beta$ sheet contains the canonical FLVRES sequence, where arginine $\beta B 5$ forms the critical electrostatic bond with two oxygen atoms in the phosphotyrosine to generate domainligand binding [6]. While this contact is central for $\mathrm{SH} 2$-phosphotyrosine interactions, carboxyl-terminal residues within the $\beta$-sheet create variable binding interfaces ("specificity pockets") that dictate ligand specificity based on the residues flanking the phosphotyrosine [7-9]. Though well characterized in terms of phosphotyrosine ligand binding, emerging reports have determined that select $\mathrm{SH} 2$ domains bind certain ligands independent of tyrosine phosphorylation $[7,10,11]$. These interactions are mediated by ligand binding to regions on the $\mathrm{SH} 2$ domain that either include or exclude participation of the phosphotyrosine binding cleft, potentially increasing the variability and complexity of $\mathrm{SH} 2$ domain function in signal relay systems.

Src and related tyrosine kinases are highly regulated enzymes where the SH2

domain plays a pivotal role in controlling kinase function. Binding of the Src SH2 
domain to the phosphorylated carboxyl-terminal tyrosine 527 is key in maintaining Src in a closed autoinhibitory state $[12,13]$. Tyrosine 527 dephosphorylation results in an open conformation, allowing the kinase $(\mathrm{SH} 1)$ domain to phosphorylate substrates. Elimination of tyrosine 527 results in constitutive kinase activation and neoplastic transformation [14]. The Src SH2 domain also potentiates kinase activity through stable binding to several tyrosine phosphorylated substrates, notably focal adhesion kinase (FAK) and p130CAS $[15,16]$. Src regulates cellular growth, division, adhesion, and motility through SH2-mediated interactions and subsequent cis and/or trans substrate tyrosine phosphorylation [17]. Elevated growth factor signaling in human cancer leads to Src hyperactivation, promoting tumor progression through increased growth and invasive potential. Increased Src activity accomplishes this by promoting tumor cell migration, invadopodia formation, and matrix metalloproteinase (MMP) activity [18]. These attributes have resulted in the development of kinase-targeted Src inhibitory compounds that are currently being evaluated for efficacy as anti-tumor and -metastatic therapeutics. [19].

Several actin-binding proteins serve as $\mathrm{Src}$ targets for $\mathrm{SH} 2$ domain binding and phosphorylation that modulate actin dynamics essential for whole and intracellular motility. Cortactin is a filamentous (F)-actin binding protein that regulates actin related protein (Arp)2/3-based actin network formation responsible for cortical actin-based membrane protrusion [20]. Src phosphorylates cortactin at three positions (Y421/466/482 in the murine form) within a proline (P)-rich domain near the carboxyl terminus [21]. Cortactin tyrosine phosphorylation coincides with cellular membrane deforming events involving cortical actin remodeling, including cell migration, pathogen 
uptake, endocytosis, osmotic shock, synaptic remodeling, cell junction regulation, and invadopodia formation [22]. Mechanistic insight to date indicates that cortactin tyrosine phosphorylation creates binding sites for $\mathrm{SH} 2$ domain-containing adaptor proteins. These include Crk during Shigella internalization [23] and Nck1 in invadopodia maturation [24]. For Nck1, additional Nck1 domains mediate the assembly of N-WASpcontaining macromolecular complexes that further enhance Arp2/3 actin network formation [25]. Src-mediated cortactin phosphorylation also enhances binding of the cortactin carboxyl-terminal SH3 domain to proline-rich domains in target proteins [26], although the molecular details of this process are currently unclear.

While the $\mathrm{SH} 2$ domain has been previously shown to be solely responsible for mediating Src association with cortactin [27], the precise $\mathrm{SH} 2 \mathrm{Src}$ interaction site on cortactin is unknown. Here we demonstrate that Src associates with cortactin through a phosphotyrosine-independent $\mathrm{SH} 2$ domain interaction involving the formation of a cystine linkage. Deletion and mutational mapping indicates that cysteine residues 112 in the 1 st and 246 in the 5th cortactin repeat represent two separate docking sites for the $\mathrm{Src} \mathrm{SH} 2$ domain. Src and cortactin form a stable redox-sensitive linkage in cells that is required for cortactin phosphorylation. Molecular modeling of the $\mathrm{Src} \mathrm{SH}_{2}$ domain shows peptides containing cortactin $\mathrm{C} 112$ and $\mathrm{C} 246$ dock within the Src phosphotyrosine-binding cleft, with cortactin cysteine residues in close proximity to Src C185 at position $\beta C 3$. Tandem mass spectroscopy of the Src SH2 domain mixed with cortactin peptides demonstrates formation of cystine bonds between Src C185 and cortactin C112 and C246. Cells containing cortactin mutants lacking C112 and C246 display reduced cortactin tyrosine phosphorylation, motility, and adhesion. Cortactin 
C112/246 is required for the formation of initial (pre-) invadopodia complexes and extracellular matrix degradation. Our results indicate that Src interacts with cortactin independent of tyrosine phosphorylation through novel cystine bonding within the Src $\mathrm{SH} 2$ domain phosphotyrosine-binding region. Sequence inspection indicates that $25 \%$ of all $\mathrm{SH} 2$ domains contain a cysteine at, or in close proximity to $\beta \mathrm{C} 3$, pointing to potential widespread usage of cystine-based $\mathrm{SH} 2$ domain interactions in numerous signaling pathways. 


\section{Results}

\section{The Src SH2 domain binds cortactin independent of tyrosine phosphorylation}

Src and other tyrosine kinases phosphorylate cortactin on tyrosines 421,466 , and $482[26,28]$. Using affinity precipitation assays with purified $\mathrm{Src} \mathrm{SH} 2, \mathrm{SH}$, and tandem $\mathrm{SH} 2 / \mathrm{SH} 3$ fusion proteins, we confirmed previous work [27] that the Src $\mathrm{SH} 2$ domain is the only region on Src responsible for binding cortactin (Fig. S1A). To determine whether the Src SH2 domain can interact with any of the primary cortactin phosphotyrosine residues, a commercial $\mathrm{SH} 2$ domain array was screened with cortactin peptides surrounding the two main Src phosphorylation targets (Y421 and Y466). Interestingly, neither phosphorylated nor unphosphorylated peptides interacted with the $\mathrm{Src} \mathrm{SH} 2$ domain, although both peptides showed phospho-dependent binding to the $\mathrm{SH} 2$ domains of the tyrosine kinases Abl and Fyn (Fig. S1B-D), both which target cortactin $[29,30]$. We pursued this finding utilizing GST-Src SH2 pull-down assays from lysates of epidermal growth factor (EGF)-stimulated cells. There was no difference between the amounts of cortactin precipitated with the Src GST-SH2 domain from EGFstimulated cells compared to non-stimulated cells, even though stimulated cells showed increased cortactin tyrosine phosphorylation (Fig. 1A). We next evaluated the direct association of GST-Src SH2 with cortactin using Far Western analysis. Cells were transfected with FLAG-tagged constructs expressing full-length cortactin (FL) or cortactin containing phenylalanine substitutions at the dominant Src phosphorylation site (Y421F;[21]) or all three Src phosphorylation sites (triple tyrosine mutant; TYM). The Src SH2 domain bound cortactin at equivalent levels from starved or EGFstimulated cells whether or not Src-targeted cortactin tyrosine residues were present 
(Fig. 1B). The same result was obtained by GST-Src SH2 affinity precipitation (Fig. 1C). To further confirm phosphotyrosine independence, we assayed bacteriallyproduced, non-phosphorylated recombinant cortactin by GST-SH2 affinity precipitation. A dose-dependent increase in cortactin binding by the $\mathrm{Src} \mathrm{SH} 2$ domain was observed above saturated GST control levels (Fig. 1D). Constitutively active Src phosphorylated recombinant cortactin (Fig. S2A) and GST-Src SH2 interacts with FAK Y397 in a phosphotyrosine-dependent manner (Fig. S2B), indicating that the assayed proteins retained correct functionality.

\section{The Src SH2 domain binds to cortactin repeat 1 and repeat 5}

To identify the cortactin region responsible Src SH2 binding, we utilized a series of deletion mutants with the systematic removal of cortactin structural domains that independently retain their respective functions [31, 32] (Fig. S3). Far Western and affinity precipitation assays indicated that GST-Src $\mathrm{SH} 2$ bound to the amino terminal (NT) half of cortactin (residues 1-330) rather than the carboxyl terminal (CT) half that contains the sites of tyrosine phosphorylation (Fig. S2C-D). Separation of the Arp2/3binding N-terminal acidic (NTA) domain from the F-actin binding repeats domain indicated that the Src SH2 domain associated with the cortactin repeats region (Fig. 1E). Serial deletion of individual cortactin repeats beginning with repeat 3 and retaining the carboxyl terminus demonstrated a significant reduction of $\mathrm{Src} \mathrm{SH} 2$ domain binding with removal of the 5th cortactin repeat (Fig. 1F). Deletion of repeat 5 in the context of the full-length cortactin protein failed to prevent $\mathrm{SH} 2$ binding (Fig. S4A), suggesting that there is at least one additional repeat that binds the $\mathrm{Src} \mathrm{SH} 2$ domain. To test this, we utilized a carboxyl terminal deletion series by Far Western analysis where each 
cortactin repeat was serially removed (Fig. S4B). Src SH2 binding was observed in all constructs containing repeat 1 . Since either set of deletion constructs cannot clearly determine that repeats 1 and 5 are the only repeats capable of $\mathrm{Src} \mathrm{SH} 2$ binding, we created chimeric constructs by adding each individual cortactin repeat in tandem amino terminal to repeat 6 in the SH2-null binding R6-CT construct (Fig. S3). Far Western assays of these constructs unambiguously established cortactin repeat 1 and repeat 5 as individual interaction regions for Src SH2 domain binding (Fig. 1G).

\section{Cysteine 112 and cysteine 246 mediate cortactin binding to the Src SH2 domain}

Sequence alignment of each cortactin repeat identified cysteine 112 in repeat 1 and cysteine 246 in repeat 5 as residues that lacked significant homology with cortactin residues in the same position (Fig. 2A). To determine if these residues were responsible for their respective cortactin repeats to bind the $\mathrm{Src} \mathrm{SH} 2$ domain, we mutated each cysteine individually to alanine in the full-length wild type molecule (WT C112A and WT C246A) and in their respective dual tandem repeat 6 chimeras (R1R6 C112A and R5R6 C246A). Far Western assays indicated that Src SH2 binding was retained in the WT C112A and WT C246A constructs but not by the R1R6 C112A and R5R6 C246A chimeric mutants (Fig. 2B-C). These data indicate that C112 and C246 function as separate, independent binding motifs for the $\mathrm{Src} \mathrm{SH} 2$ domain. Given the duplicity of these SH2 binding sites, we mutated cysteine 112 and 246 to alanine in the WT cortactin construct (double cysteine mutant; DCM) and tested Src SH2 binding by Far Western analysis and affinity precipitation. Mutation of both cysteine residues 
abolished Src SH2 domain binding (Fig. 2D-E). These data indicate that cysteine 112 and 246 are required for Src SH2 association with cortactin.

\section{Molecular modeling of cortactin C112 and C246 pentapeptides with the Src SH2 domain}

Given the apparent unique binding requirements for cortactin to the Src SH2 domain, we conducted molecular modeling with cortactin C112 and C246 peptides to gain mechanistic insight into how the cortactin cysteine residues might interact with the $\mathrm{SH} 2$ domain. The SH2 domain of Src was obtained from the published crystal structure [33]. As a positive control, a pentapeptide encompassing Src pY527 was docked to Src SH2. The peptide was predicted to dock in the phospho-tyrosine binding pocket in close proximity with the $\beta B 5$ arginine 175 (Fig. 3A). The phosphotyrosine in the docked structure displayed the identical $2.72 \AA$ distance between R175 and the pY527 phosphate group obtained by co-crystallization and NMR analysis [34]. Analogous cortactin and corresponding control pentapeptides, encompassing C112, A112, C246, and $\mathrm{A} 246$, docked in the presence the SH2/pY527 complex failed to produce sufficient binding energies predictive of cortactin binding. This suggested that the tested cortactin peptides might bind within the Src SH2 phosphotyrosine binding cavity. To test this, similar docking studies were conducted in the absence of the pY527 Src peptide. Under these conditions all cortactin peptides were predicted to bind within the Src SH2 phospho-tyrosine binding cleft (Fig. 3A). Interestingly, the cysteine/alanine residues within each cortactin peptide dock in close proximity to Src C185 in the $\beta C 3$ position 
(C112; $5.83 \AA$, A112; $5.96 \AA, C 246 ; 3.56 \AA$, A246; $3.17 \AA$ ) (Fig. 3A). These data suggested the possibility that cortactin C112 or C246 might form a cystine bond with Src C185 to mediate Src-cortactin binding. The predicted distances between cortactin C112/246 and Src C185 are greater than the typical $\sim 2 \AA$ distance for cystine bonds determined by Raman spectroscopy [35]. This difference is attributed to the inability of the modeling program to construct disulfide bonds, as well as program-predicted deprotonation of all cysteines in the assay, imparting negative charges to C112/246 and Src C185. In spite of the introduced repulsive effects, the predicted binding energies of the $\mathrm{C} 112 / \mathrm{C} 246$ cortactin peptides are lower than the A112/A246 peptides by $6-8$ $\mathrm{kcal} / \mathrm{mole}$, suggesting a more favorable binding affinity for the cysteine-containing cortactin peptides (Fig. 3B). Although nearly 50\% weaker than predicted pY527 peptide binding, the presence and location of cysteine at cortactin 112 and 246 within the Src $\mathrm{SH} 2$ binding pocket suggested a potentially favorable interaction with the Src SH2 domain. These data implicate Src $\mathrm{C} 185$ as a key residue within the SH2 domain responsible for cortactin binding through cystine bond formation.

\section{Cystine bonding mediates Src SH2 binding to cortactin}

To evaluate if cystine bonding mediates Src binding to cortactin, the association of endogenous Src and cortactin was determined under differential redox conditions. Cortactin specifically co-immunoprecipitated with Src from UMSCC1 HNSCC cells migrated at the typical $70 \mathrm{kDa} M_{\mathrm{r}}$ when immune complexes were incubated with 2mercaptoethanol and analyzed by SDS-PAGE and Western blotting (Fig. 4A). 
However, cortactin $M_{r}$ was severely retarded when immunoprecipitates were prepared in the absence of reductant, banding as several higher molecular weight species at 90 , 120 and $165 \mathrm{kDa}$ (Fig. 4A). A similar pattern was observed for Src in cortactin immunoprecipitates (Fig. 4B), indicating that a subset of Src and cortactin complex under oxidative conditions required for cystine bonding. Cortactin phosphorylation by Src is also oxidation dependent, as incubation of Src immunoprecipitates with purified recombinant cortactin in the presence of dithiothreitol (DTT) prevents Src phosphorylation of cortactin Y421 (Fig. 4C).

Since Src C185 is predicted to be the key residue mediating Src-cortactin bonding, Src C185 was mutated to alanine (C185A) and the recombinant $\mathrm{SH} 2$ domain assayed for cortactin binding. C185A eliminated WT cortactin binding but did not perturb phosphotyrosine-dependent binding to FAK (Fig. 4D-E). Mutation of the arginine responsible for phosphotyrosine binding (R175) to alanine did not alter binding to WT or NT cortactin, verifying phosphotyrosine independence (Fig. S5). Reduction of the Src SH2 domain with DTT did not impact binding to FAK but abolished cortactin binding (Fig. 4D).

To directly verify the existence of cystine bonding between Src C185 and cortactin C112/246, we incubated the GST-Src SH2 domain with saturating amounts of 7 mer peptides encompassing cortactin C112 and C246 in the absence of reducing agents. Control (GST-SH2 alone) and SH2-cortactin peptide mixtures were subjected to LCMS/MS to identify cystine bonding between the Src SH2 domain and each cortactin peptide. The predicted trypsin digest product from the Src SH2 domain containing C185 is a 14 mer peptide with C185 in position four. The fragment composition and 
mass for the Src SH2 domain alone and cystine-bound to the digested C112 and C246 cortactin peptides are shown in Fig. 5A. Fragmentation and subsequent spectral sequence analysis indicated that cystine bonding occurred between Src C185 and cortactin C112 or C246, as evidenced by mass shifts of the b4 ions in the Src C185 peptide fragment in experiments containing cortactin C112 (Fig. 5B vs 5C) and C246 (Fig. 5B vs 5D) peptides. Analogous shifts were observed upon inspection of the y11 ions for each experimental condition (Fig. S6). Complete sequence coverage of the $\mathrm{SH} 2$ domain fragment from the SH2-C112 and $\mathrm{SH} 2-\mathrm{C} 246$ experiments in the b- and $\mathrm{y}$ planes yielded observed peptide masses identical or very close to the predicted mass at each ion parameter (Table 1). These data collectively indicate that Src C185 is capable of forming cystine bonds with cortactin C112 and C246 under oxidizing conditions, representing a novel mode of interaction between an $\mathrm{SH} 2$ domain and its respective target ligand.

\section{Cortactin C112/C246 are required for cortactin tyrosine phosphorylation and Src- based cellular processes}

To evaluate the impact of cortactin C112/C246 on biochemical and cellular functions involving Src, we initially determined the ability of Src to phosphorylate cortactin DCM. While expression of cortactin WT with WT Src (c-Src) in murine fibroblasts lacking Src, Yes, and Fyn (SYF) demonstrated robust cortactin Y421 phosphorylation, expression of cortactin DCM with c-Src resulted in a complete lack of cortactin tyrosine 421 phosphorylation (Fig. 6A). Expression of cortactin DCM in MTLn3 cells resulted in 
diminished Y421 phosphorylation on par with the phosphorylation-null cortactin TYM construct (Fig. 6B), indicating that cortactin C112/C246 are essential for Src-mediated cortactin phosphorylation. We next analyzed the effect of cortactin DCM on cell migration and adhesion, two processes that involve Src activation and downstream cortactin tyrosine phosphorylation. Cortactin DCM expression in 1483 head and neck squamous cell carcinoma (HNSCC) cells with stable endogenous cortactin knockdown (Fig. S7A) failed to rescue adhesion and migration to levels similar to control or WT cortactin, and were equivalent to knockdown (sh) and TYM expressing cells (Fig. 6C-D). Finally, we analyzed how cortactin DCM affects invadopodia formation and extracellular matrix degradation, processes dependent on Src and cortactin. Control (Ctl) OSC19 HNSCC cells spontaneously form numerous invadopodia that focally degrade labeled gelatin matrices. Invadopodia were completely absent in cortactin knockdown (sh) cells (Fig. 6E) as previously shown [36, 37]. Re-expression of WT cortactin rescued the knockdown phenotype, restoring matrix degradation to control levels. However, rescue of cortactin sh cells with cortactin TYM resulted in the formation of invadopodia structures with limited matrix degradation ability. These structures are likely preinvadopodia as previously described $[24,38]$. Rescue of cortactin sh cells with cortactin DCM failed to restore invadopodia/pre-invadopodia formation and geletin degradation. These results indicated that the genesis of pre-invadopodia requires initial binding of Src to cortactin (Fig. 6E). The DCM protein correctly localizes within lamellipodia (Fig. $6 \mathrm{E})$, suggesting that the $\mathrm{C} 112 / 246 \mathrm{~A}$ mutations do not deleteriously impact cortactin structure or alter proper cortactin subcellular localization. C112/246A does not significantly alter binding to F-actin as determined by F-actin co-sedimentation assays 
(Fig. S7B, C). Collectively these data indicate that cystine-mediated Src binding is required for Src phosphorylation of cortactin and regulation of cellular events required for pro-motile and invasive activity. 


\section{Discussion}

While $\mathrm{SH} 2$ domains have been shown to interact with ligands through phosphorylation-dependent and -independent mechanisms, binding to phosphotyrosine residues within target proteins is the predominant mode of interaction, having been characterized at the structural and thermodynamic levels for numerous ligand/domain pairs $[39,40]$. SH2 domains are present in proteins that mediate most cellular functions and provide a wide combinatorial variety for selective intracellular signal transfer [2]. The identification of a cystine-based, tyrosine phosphorylation-independent interaction between the phosphotyrosine binding interface of the $\mathrm{Src} \mathrm{SH} 2$ domain with cortactin increases the potential ability for Src to interact with substrate ligands in a previously unrealized manner, expanding the repertoire and complexity of $\mathrm{Src} \mathrm{SH} 2$ domain interactions in signal transduction.

Evidence from our analysis of $\mathrm{Src} \mathrm{SH} 2$ domain binding to cortactin indicates that Src C185 forms a cystine bond with C112 and/or C246 in the cortactin repeats region that is critical for mediating cortactin binding, tyrosine phosphorylation, and downstream cellular events. When bound to tyrosine phosphorylated ligands, C185 lies in close proximity to the phosphate group within the binding pocket, where the intrinsic repulsive nature of the deprotonated $\mathrm{C} 185$ facilitates release of pY527 from the SH2 domain, assisting in relieving the kinase from the autoinhibited state [41]. Our data provides an additional and alternative function for Src C185 in docking to cortactin, where cystine bonding to C112 or C246 mediates association with activated Src. Structural studies

indicate cortactin exists as a partially globular protein, where the repeats are in a paraordered molten globule state that is highly dynamic in solution $[42,43]$. Biophysical 
and biochemical analysis indicates that the carboxyl terminus folds back onto the amino terminal region, suggesting that the protein resides in a "closed" conformation as supported by previously observed conformational isomers $[44,45]$. C112 and C246 can be crosslinked to each other, suggesting they are exposed and either lie in close proximity or are dynamically brought together [42, 43]. The relatively unstructured nature of the cortactin repeats therefore provides the flexibility and accessibility of C112 and $\mathrm{C} 246$ to freely dock with the Src SH2 domain, allowing cystine bonding to occur. The nearness of the cortactin repeats region to the carboxyl terminal target tyrosine residues would allow for $\mathrm{SH}$ 2-directed Src binding to the cortactin amino terminus, followed by subsequent processive phosphorylation of Y421/466/482 within the P-rich domain [46]. In conjunction with Erk1/2 phosphorylation at S405/418 [45, 47], cortactin is predicted to assume an open conformation, exposing the phosphotyrosine residues for association with SH2 containing adaptor proteins [25] or Abl-family kinases [48] (Fig. 7A). The net effect of Src-based phosphorylation would enhance actin dynamics through adaptor protein interactions or by maintaining activation of $\mathrm{SH}$-bound $\mathrm{Abl}$ or Arg, promoting phosphorylation of cortactin and neighboring target proteins [48, 49]. This model could be regulated by additional binding interactions and modifications that involve the cortactin repeats region. While mutation of the C112/246 Src docking sites does not alter F-actin binding, other modifications to the cortactin repeats region do modulate F-actin binding, including binding of phosphatidylinositol 4,5-bisphosphate [50], acetylation of the cortactin repeats [51], and phosphorylation of S113 in the first repeat by PAK [52]. Whether these events impact the association of Src with cortactin remains to be determined. 
Given the unprecedented nature of $\mathrm{SH} 2$ domains utilizing cystine bonding for binding ligands, we conducted a phylogenetic analysis of sequences containing cortactin C112/C246 and Src C185 in species ranging from Homo sapiens to Suberites domuncula (Fig. 7B). This analysis shows that the cortactin cysteine 112 equivalent appears in accord with the equivalent Src C185 residue in Danio rerio and is conserved throughout higher species. Cortactin C246 first appears in Xenopus laevis and is present in all higher organisms, collectively indicating co-conservation of the Src and cortactin cysteines in vertebrates.

There is mounting evidence to date that oxidative-based cystine and disulfide bonding occurs within and between multiple different cytoplasmic proteins during conditions of cytoplasmic oxidative stress as a mechanism that serves to regulate protein functionality [53-55]. Src activity can be regulated by reactive oxygen species (ROS) [56], which have a broad impact on multiple cellular processes that utilize oxidative signaling [57]. Specifically, ROS-induced cysteine thiol oxidation of Src C245 in the $\mathrm{SH} 2$ domain and $\mathrm{C} 487$ in the kinase domain has been proposed to sustain Src activity by cystine bonding between these residues, maintaining Src in an open conformation to promote kinase activity [58]. The proximity of Src C185 to cortactin C112/246 docked within the SH2 phosphotyrosine binding cleft provides the molecular setting that allows for a similar redox-mediated oxidation and consequential cystine bonding between the two proteins. Oxidative regulation of Src-cortactin binding is supported by increased Src-based cortactin phosphorylation in cells treated with hydrogen peroxide [59]. The requirement for cortactin C112/246 in tumor cell motility, 
adhesion, and invadopodia formation is in line with oxidative Src regulation utilized in these processes $[57,60]$. Furthermore, work in invadopodia has shown that localized ROS production generated by the nicotinamide adenine dinucleotide phosphate (NADPH) oxidase (Nox) system occurs through association of the invadopodial protein Tks5 with the Nox component p22 ${ }^{\text {phox }}[61]$. Tks5 generated ROS promotes invadopodia formation and generates a feed forward loop through ROS-induced Src activity by suppression of the Src inactivating phosphatase PTP-PEST [61] and potentially by sustaining cystine-based Src activity as described. ROS production in invadopodia would therefore facilitate oxidation of Src C185 and cortactin C112/246, promoting cystine bonding and maintenance of the Src-cortactin complex at sites of invadopodia formation. Since pre-invadopodia can form in the absence of cortactin phosphorylation [24], we speculate that the initial event for pre-invadopodia formation involves oxidativebased cystine binding of Src to cortactin. Once established, successive cycles of Src activity coupled with dynamic cortactin phosphorylation/dephosphorylation promotes establishment of $\mathrm{SH} 2$ protein-based actin regulatory complexes to propagate actin filament production and ECM matrix degradation [24, 38]. While recent work in MDMBA-231 cells suggests that Arg, and not Src, is responsible for regulating cortactin phosphorylation during invadopodia formation [62], our data in HNSCC cells suggests that the tyrosine-independent binding event between Src and cortactin is the essential trigger for pre-invadopodia production. Determination of whether these differences are tumor specific remains to be resolved. 
Cystine formation between Src and cortactin must be transient and reversible, since the $\mathrm{Src}$ SH2 domain does not stably interact with cortactin as opposed to other phospho-tyrosine ligands [63, 64]. Also, dynamic cycling of Src kinase activity is required for invadopodia maturation $[24,47]$. Cytoplasmic glutathione likely plays a part in providing the reductive counterbalance to Tks5-generated ROS production, providing the necessary redox balance to generate and reduce thiolate anions at $\mathrm{pH}$ levels present in invadopodia $[53,65]$. Another possibility is that Src and cortactin binding may be downregulated through the action of thiol reductases, although the subcellular localization of these enzymes has not been extensively evaluated.

Collectively our data indicates that the $\mathrm{Src} \mathrm{SH} 2$ phosphotyrosine binding cleft is capable of phosphotyrosine and cystine dependent and independent interactions. The dual specificity of this $\mathrm{SH} 2$ subregion may be utilized to increase the selectivity between different classes of Src substrates (Fig. 7C) and may warrant reinvestigation of Src SH2-targeting compounds designed to react with $\mathrm{C} 185$ as alternative therapeutic strategy to the current class of kinase targeted Src inhibitors being evaluated as anticancer therapeutics in clinical trials [66]. Ablating C185 reactivity, while retaining phosphotyosine binding, would selectively impair the Src-cortactin axis without impeding essential phosphorylation-based $\mathrm{SH} 2$ domain-ligand interactions. This rationale may be warranted in HNSCC, where Src activating epidermal growth factor receptor (EGFR) and cortactin are frequently amplified and overexpressed, corresponding with poor patient outcome $[67,68]$. 
Database analysis indicates that Src is the only $\mathrm{SH} 2$ domain-containing cytoplasmic tyrosine kinase in its class that harbors a cysteine residue within the $\mathrm{SH} 2$ domain (Fig. 7D). However, there are thirty additional $\mathrm{SH} 2$ domains (25\% of the total known number) that contain cysteine residues within the phosphotyrosine binding region in close proximity to the Src $\beta C 3$ (Fig. 7D). While untested, this indicates that $\mathrm{SH} 2$ domains containing cysteine residues within this region may broadly utilize cystine bonding to select ligands. This alternate $\mathrm{SH} 2$ signaling mode may be commonplace in conditions of high intracellular ROS due to environmental stress (e.g.; heat, ionizating radiation, ultraviolet light), as well as in hypoxic tumors. Alternatively, cystine-based $\mathrm{SH} 2$ signaling may serve as a "backup" mode of preserving domain-ligand binding to ensure that essential survival signals are mediated in conditions of low ATP availability, where tyrosine kinase signaling might be compromised (i.e.; Warburg effect). While future investigation is required to examine these possibilities, our results demonstrating cystine bonding between Src and cortactin represent a new paradigm for $\mathrm{SH} 2$ domain function in mediating domain-based signal transduction. 


\section{Materials and Methods}

\section{Cell Culture}

MTLn3 cells were maintained in alpha-minimal essential medium ( $\alpha-M E M)$ (Mediatech) with 10\% FBS (Hyclone) in a 5\% $\mathrm{CO}_{2}$ humidified atmosphere. SYF, 293T, 1483, and OSC19 cell lines were maintained in Dulbecco's modified Eagles medium (DMEM) (Mediatech) with 10\% FBS.

\section{Generation of Plasmids}

Cortactin truncation, Y421, and TYM mutants were previously described [31, 46]. Tandem repeat chimeric mutants were generated by subcloning each individual repeat containing flanking BamH1 and Xho1 restriction sites with the Xho1/EcoR1 R6-CT fragment into BamH1/EcoR1 digested pcDNA3 FLAG-2AB. CFP-Src was produced by subcloning the Src containing Xho1/BamH1 fragment from GFP-Src (a gift from Margaret Frame) into pECFP-N1 (Clontech). FAK WT and Y397F were subcloned into FLAG-2AB using BamH1 and EcoR1 restriction sites. Site-directed mutagenesis of cortactin constructs was performed using the QuickChange II $^{\mathrm{TM}}$ Site-directed mutagenesis kit (Agilent Technologies) according to the manufacturer's protocol.

\section{Virus Production and Generation of Stable Cell Lines}

Stable knockdown of cortactin in 1483 and OSC19 cells was achieved by lentiviral transduction using short hairpin lentiviral constructs from Open Biosystems (1483: TRCN0000040275, OSC19: TRCN0000040275 and TRCN0000040273). Complete cortactin knockdown in OSC19 cells was achieved by subsequent transfection of 
cortactin-targeting siRNA (ON-TARGETplus SMARTpool cat\# L-010508-00-0020, Dharmacon).

\section{Confocal Microscopy and Gelatin Degradation Assay}

Immunofluorescent labeling, confocal microscopy, preparation of fluorescently-labeled gelatin coated coverslips, and ECM degradation assays were conducted as previously described [69].

\section{Peptide Synthesis and SH2 Domain Array Screening}

Peptides were synthesized by Macromolecular Resources at Colorado State University. Screening of Transignal ${ }^{\mathrm{TM}} \mathrm{SH} 2$ Domain Arrays (Panomics Cat. \# MA3040) was conducted using $1.0 \mathrm{mg}$ of each cortactin peptide according to the manufacturer's protocol.

\section{Fusion Protein Purification}

Purification of recombinant proteins were performed as described previously [27].

\section{Cell Transfection, Western Blotting, and Immunoprecipitation}

Plasmid transfection, Western blotting, and immunoprecipitation were performed as described [70].

\section{Far Western Assays}

Initially, cells transfected with FLAG-tagged cortactin plasmids were lysed and immunoprecipitated using EZ-View anti-FLAG resin (Invitrogen). Immune complexes were separated by SDS-PAGE, transferred to nitrocellulose membranes and proteins 
renatured in TBS-Tween containing 5\% nonfat milk. Far Western binding with Src SH2 domain constructs was conducted essentially as described [71], except $2 \mathrm{~h}$ incubations with 50-200 $\mu \mathrm{g}$ of fusion protein were used. In reduction experiments (Fig. 4D), $1 \mathrm{mM}$ DTT was added to the incubation solution containing the GST-Src SH2 domain.

\section{Affinity Precipitation}

$\mathrm{SH} 2$ domain precipitations were conducted as described [27], except 100-200 $\mu \mathrm{g}$ of recombinant fusion protein and 1.0-1.5 mg of cell lysate were used for each assay.

\section{Electric Cell-Substrate Impedance Sensing (ECIS)}

ECIS was performed as described [70].

\section{F-actin Cosedimentation Assay}

F-actin cosedimentation assays were performed as described [31].

\section{Statistical Analysis}

For standard errors (d-SE), variances were pooled using residual standard error from one-way ANOVA without the intercept. Delta-method approximation was used for all confidence bands (Fig. 6). Transformations performed in ANOVA used standard statistical model evaluation tools.

\section{Molecular Modeling}

Docking studies were performed using the program eHiTS (SymBioSys Toronto, CA). Prior to docking, the hydrogens were added to the crystal structure PDBID: 1FMK [33] of the $\mathrm{Src} \mathrm{SH} 2$ domain, solvated, and ions to neutralize the charge were added. The 
entire structure was then relaxed by running molecular dynamics (300 ps) using Amber (ver. 10) [72]. The cortactin peptides were built de novo in an extended conformation using Insight II (Accelrys, San Diego, CA) and each allowed to relax through 2500 steps of steepest descent molecular mechanics. The resulting structures were then docked. To obtain binding energies, molecular dynamics simulations were performed for $300 \mathrm{ps}$ for: 1) each cortactin peptide in complex with the $\mathrm{Src} \mathrm{SH} 2$ receptor, with docking results used as the starting structure; 2) the Src SH2 receptor alone and 3) each cortactin ligand alone. The resulting average potential energies calculated from the molecular dynamics simulation were then used to calculate the binding energy for each cortactin ligand to the $\mathrm{SH} 2$ receptor as the energy of the complex minus the energy of the Src $\mathrm{SH} 2$ receptor alone minus the energy of the cortactin ligand alone. Parameters for the phosphotyrosine were used [73].

\section{Mass Spectrometry}

Purified GST-Src SH2 domain was incubated alone or mixed with a 10-fold molar excess of cortactin C112 peptide (SKHCSQV) or C246 peptide (QDKCALG) (Anaspec) in TBS ( $\mathrm{pH} 7.2)$ for $2 \mathrm{~h}$ at room temperature. GST-Src SH2 samples (40 $\square \mathrm{g}$ ) were subsequently digested with trypsin (Promega) at a 1:50 (wt/wt) ratio in $1 \mathrm{mM}$ Tris, 150 $\mathrm{mM} \mathrm{NaCl}$ (TBS), $\mathrm{pH} 7.2$ overnight at $37^{\circ} \mathrm{C}$. Digested samples were frozen at $-80^{\circ} \mathrm{C}$ and lyophilized to remove solvents. Tryptic peptides were separated using an Acquity UPLC $®$ System (Waters). Samples were separated using a reverse-phase Acquity BEH C18 $1.7 \mathrm{~mm}, 1.0$ x $50 \mathrm{~mm}$ column (Waters) directly coupled to a Waters Synapt ${ }^{\circledR}$ G2 HDMS mass spectrometer. Data were acquired using time of flight (TOF) MS mode, which provides a comprehensive catalog of information for both precursor and 
fragment ions in a single analysis. A customized database was created using the GSTSrc SH2 sequence, and the resulting precursor masses and MS/MS spectra were searched against the database using Biopharmalynx 3.1 (Waters). To evaluate cortactin $\mathrm{C} 112$ and $\mathrm{C} 246$ peptide binding to the $\mathrm{Src}$ SH2 domain, custom posttranslational modifications were specified in Biopharmalynx based on monoisotopic masses for the C112 and C246 peptides binding at cysteine residues to Src C185 through disulfide bond formation. Extracted ion chromatograms (XIC) were obtained using MassLynx 2.2 (Waters).

\section{Accession Numbers for Phylogenetic Sequence Analysis}

Respective Genebank accession numbers for cortactin and Src are as follows: Homo sapiens AAH08799.1, NP_005408.1; Canis familiaris XP_851317.1, XP_865870.1; Mus musculus AAA19689.1, AAX90616.1; Gallus gallus Q01406.1, NP_990788.2; Xenopus laevis BAB79435.1, AAH45134.1; Danio rerio NP_001004121.1, AAI65380.1; Tetraodon nigroviridis CAF92908.1, CAG10364.1; Drosophila melanogaster NP_524426.2, NP_001189051.1; Anopheles gambiae XP_557457.3, XP_316537.2;

Loa loa XP_003142854.1, EFO14749.1; Stongylocentrotus purpuratus AAD08655.1, ACI14304.1; Suberites domuncula CAC80140.1, AAT67598.1.

\section{SH2 Domain Alignments}

Twenty amino acids within the phosphotyrosine binding pocket of the $\mathrm{Src} \mathrm{SH} 2$ domain beginning with the FLVRES sequence were aligned relative to Src C185, which was used as the zero residue reference point. SH2 domain sequence data were obtained 
from the University of Chicago Nash Laboratory $\mathrm{SH} 2$ domain database (http://sh2.uchicago.edu/clustalalignment.html).

\section{Antibodies}

Antibodies for immunoblotting were as follows: anti-cortactin (4F11), 1:1000; anti-pY421 cortactin (Invitrogen), 1:1000; anti-FLAG and anti-GST (Millipore), 1:1000; anti-GFP (JL8; Clontech), 1:1000; anti-phosphotyrosine (BD Transduction), 1:1000. Antibodies for immunofluorescent labeling were used as described [69].

\section{Acknowledgements}

We thank Mike Schaller, members of the Weed laboratory and MBR Cancer Center for advice and suggestions, M. Auble for early experimental help, and B. Robke for peptide synthesis. Imaging experiments were performed in the West Virginia University Microscope Imaging Facility, which is supported in part by the Mary Babb Randolph Cancer Center and NIH grant P30 RR032138/GM103488. This work was supported by NIH grants DE014364, DE014578 and RR16440 to SAW, by the Mary Babb Randolph Cancer Center, and by the West Virginia University Department of Neurobiology and Anatomy. 


\section{References}

1. Koch, C.A., et al., SH2 and SH3 domains: elements that control interactions of cytoplasmic signaling proteins. Science, 1991. 252(5006): p. 668-74.

2. Liu, B.A., et al., The human and mouse complement of SH2 domain proteins-establishing the boundaries of phosphotyrosine signaling. Mol Cell, 2006. 22(6): p. 851-68.

3. Songyang, Z., et al., SH2 domains recognize specific phosphopeptide sequences. Cell, 1993. 72(5): p. 767-78.

4. Machida, K., et al., High-throughput phosphotyrosine profiling using SH2 domains. Mol Cell, 2007. 26(6): p. 899-915.

5. Pawson, T., G.D. Gish, and P. Nash, SH2 domains, interaction modules and cellular wiring. Trends Cell Biol, 2001. 11(12): p. 504-11.

6. Kuriyan, J. and D. Cowburn, Modular peptide recognition domains in eukaryotic signaling. Annu Rev Biophys Biomol Struct, 1997. 26: p. 259-88.

7. Bae, J.H., et al., The selectivity of receptor tyrosine kinase signaling is controlled by a secondary SH2 domain binding site. Cell, 2009. 138(3): p. 514-24.

8. Muller, S. and S. Knapp, Out of the box binding determines specificity of SH2 domain interaction. Structure, 2009. 17(8): p. 1040-1.

9. Yaffe, M.B., Phosphotyrosine-binding domains in signal transduction. Nat Rev Mol Cell Biol, 2002. 3(3): p. 177-86.

10. King, T.R., et al., Using a phage display library to identify basic residues in A-Raf required to mediate binding to the Src homology 2 domains of the p85 subunit of phosphatidylinositol 3'kinase. J Biol Chem, 2000. 275(46): p. 36450-6.

11. Liao, Y.C., et al., The phosphotyrosine-independent interaction of DLC-1 and the SH2 domain of cten regulates focal adhesion localization and growth suppression activity of DLC-1. J Cell Biol, 2007. 176(1): p. 43-9.

12. $\mathrm{Xu}, \mathrm{W}$., et al., Crystal structures of c-Src reveal features of its autoinhibitory mechanism. Mol Cell, 1999. 3(5): p. 629-38.

13. Okada, M. and H. Nakagawa, A protein tyrosine kinase involved in regulation of pp60c-src function. J Biol Chem, 1989. 264(35): p. 20886-93.

14. Yeatman, T.J., A renaissance for SRC. Nat Rev Cancer, 2004. 4(6): p. 470-80.

15. Schaller, M.D., et al., Autophosphorylation of the focal adhesion kinase, pp125FAK, directs SH2-dependent binding of pp60src. Mol Cell Biol, 1994. 14(3): p. 1680-8.

16. Burnham, M.R., et al., Regulation of c-SRC activity and function by the adapter protein CAS. Mol Cell Biol, 2000. 20(16): p. 5865-78.

17. Brown, M.T. and J.A. Cooper, Regulation, substrates and functions of src. Biochim Biophys Acta, 1996. 1287(2-3): p. 121-49.

18. Guarino, M., Src signaling in cancer invasion. J Cell Physiol, 2010. 223(1): p. 14-26.

19. Elsberger, B., et al., Is Src a viable target for treating solid tumours? Curr Cancer Drug Targets, 2010. 10(7): p. 683-94.

20. Kirkbride, K.C., et al., Cortactin: A multifunctional regulator of cellular invasiveness. Cell Adh Migr, 2011. 5(2).

21. Huang, C., et al., The role of tyrosine phosphorylation of cortactin in the locomotion of endothelial cells. J Biol Chem, 1998. 273(40): p. 25770-6.

22. Cosen-Binker, L.I. and A. Kapus, Cortactin: the gray eminence of the cytoskeleton. Physiology (Bethesda), 2006. 21: p. 352-61.

23. Bougneres, L., et al., Cortactin and Crk cooperate to trigger actin polymerization during Shigella invasion of epithelial cells. J Cell Biol, 2004. 166(2): p. 225-35. 
24. Oser, M., et al., Cortactin regulates cofilin and $N$-WASp activities to control the stages of invadopodium assembly and maturation. J Cell Biol, 2009. 186(4): p. 571-87.

25. Tehrani, S., et al., Src phosphorylation of cortactin enhances actin assembly. Proc Natl Acad Sci U S A, 2007. 104(29): p. 11933-8.

26. Ammer, A.G. and S.A. Weed, Cortactin branches out: roles in regulating protrusive actin dynamics. Cell Motil Cytoskeleton, 2008. 65(9): p. 687-707.

27. Okamura, H. and M.D. Resh, p80/85 cortactin associates with the Src SH2 domain and colocalizes with v-Src in transformed cells. J Biol Chem, 1995. 270(44): p. 26613-8.

28. Daly, R.J., Cortactin signalling and dynamic actin networks. Biochem J, 2004. 382(Pt 1): p. 1325.

29. Huang, J., et al., Cooperative roles of Fyn and cortactin in cell migration of metastatic murine melanoma. J Biol Chem, 2003. 278(48): p. 48367-76.

30. Boyle, S.N., et al., A critical role for cortactin phosphorylation by Abl-family kinases in PDGFinduced dorsal-wave formation. Curr Biol, 2007. 17(5): p. 445-51.

31. Weed, S.A., et al., Cortactin localization to sites of actin assembly in lamellipodia requires interactions with F-actin and the Arp2/3 complex. J Cell Biol, 2000. 151(1): p. 29-40.

32. Weaver, A.M., et al., Cortactin promotes and stabilizes Arp2/3-induced actin filament network formation. Curr Biol, 2001. 11(5): p. 370-4.

33. Xu, W., S.C. Harrison, and M.J. Eck, Three-dimensional structure of the tyrosine kinase c-Src. Nature, 1997. 385(6617): p. 595-602.

34. Waksman, G., et al., Crystal structure of the phosphotyrosine recognition domain SH2 of v-src complexed with tyrosine-phosphorylated peptides. Nature, 1992. 358(6388): p. 646-53.

35. Van Wart, H.E., et al., Disulfide bond dihedral angles from Raman spectroscopy. Proc Natl Acad Sci U S A, 1973. 70(9): p. 2619-23.

36. Artym, V.V., et al., Dynamic interactions of cortactin and membrane type 1 matrix metalloproteinase at invadopodia: defining the stages of invadopodia formation and function. Cancer Res, 2006. 66(6): p. 3034-43.

37. Clark, E.S., et al., Cortactin is an essential regulator of matrix metalloproteinase secretion and extracellular matrix degradation in invadopodia. Cancer Res, 2007. 67(9): p. 4227-35.

38. Kelley, L.C., et al., Oncogenic Src requires a wild-type counterpart to regulate invadopodia maturation. J Cell Sci, 2010. 123(Pt 22): p. 3923-32.

39. Pawson, T., Specificity in signal transduction: from phosphotyrosine-SH2 domain interactions to complex cellular systems. Cell, 2004. 116(2): p. 191-203.

40. Schlessinger, J. and M.A. Lemmon, SH2 and PTB domains in tyrosine kinase signaling. Sci STKE, 2003. 2003(191): p. RE12.

41. Bradshaw, J.M., V. Mitaxov, and G. Waksman, Investigation of phosphotyrosine recognition by the SH2 domain of the Src kinase. J Mol Biol, 1999. 293(4): p. 971-85.

42. Cowieson, N.P., et al., Cortactin adopts a globular conformation and bundles actin into sheets. J Biol Chem, 2008. 283(23): p. 16187-93.

43. Shvetsov, A., et al., The actin-binding domain of cortactin is dynamic and unstructured and affects lateral and longitudinal contacts in F-actin. Cell Motil Cytoskeleton, 2009. 66(2): p. 90-8.

44. Huang, C., et al., Down-regulation of the filamentous actin cross-linking activity of cortactin by Src-mediated tyrosine phosphorylation. J Biol Chem, 1997. 272(21): p. 13911-5.

45. Campbell, D.H., R.L. Sutherland, and R.J. Daly, Signaling pathways and structural domains required for phosphorylation of EMS1/cortactin. Cancer Res, 1999. 59(20): p. 5376-85.

46. Head, J.A., et al., Cortactin tyrosine phosphorylation requires Rac1 activity and association with the cortical actin cytoskeleton. Mol Biol Cell, 2003. 14(8): p. 3216-29. 
47. Kelley, L.C., et al., Cortactin phosphorylated by ERK1/2 localizes to sites of dynamic actin regulation and is required for carcinoma lamellipodia persistence. PLoS One, 2010. 5(11): p. e13847.

48. Lapetina, S., et al., Arg interacts with cortactin to promote adhesion-dependent cell edge protrusion. J Cell Biol, 2009. 185(3): p. 503-19.

49. Oser, M., et al., Specific tyrosine phosphorylation sites on cortactin regulate Nck1-dependent actin polymerization in invadopodia. J Cell Sci, 2010. 123(Pt 21): p. 3662-73.

50. He, H., et al., Role of phosphatidylinositol 4,5-bisphosphate in Ras/Rac-induced disruption of the cortactin-actomyosin II complex and malignant transformation. Mol Cell Biol, 1998. 18(7): p. 3829-37.

51. Zhang, X., et al., HDAC6 modulates cell motility by altering the acetylation level of cortactin. Mol Cell, 2007. 27(2): p. 197-213.

52. Webb, B.A., et al., Phosphorylation of cortactin by p21-activated kinase. Arch Biochem Biophys, 2006. 456(2): p. 183-93.

53. Cumming, R.C., et al., Protein disulfide bond formation in the cytoplasm during oxidative stress. J Biol Chem, 2004. 279(21): p. 21749-58.

54. Cumming, R.C. and D. Schubert, Amyloid-beta induces disulfide bonding and aggregation of GAPDH in Alzheimer's disease. FASEB J, 2005. 19(14): p. 2060-2.

55. Cumming, R.C., Analysis of global and specific changes in the disulfide proteome using redox two-dimensional polyacrylamide gel electrophoresis. Methods Mol Biol, 2009. 476: p. 160-74.

56. Sun, G. and D.J. Kemble, To $C$ or not to C: direct and indirect redox regulation of Src protein tyrosine kinase. Cell Cycle, 2009. 8(15): p. 2353-5.

57. Giannoni, E., M.L. Taddei, and P. Chiarugi, Src redox regulation: again in the front line. Free Radic Biol Med, 2010. 49(4): p. 516-27.

58. Giannoni, E., et al., Intracellular reactive oxygen species activate Src tyrosine kinase during cell adhesion and anchorage-dependent cell growth. Mol Cell Biol, 2005. 25(15): p. 6391-403.

59. Li, Y., J. Liu, and X. Zhan, Tyrosine phosphorylation of cortactin is required for H2O2-mediated injury of human endothelial cells. J Biol Chem, 2000. 275(47): p. 37187-93.

60. Weaver, A.M., Regulation of cancer invasion by reactive oxygen species and Tks family scaffold proteins. Sci Signal, 2009. 2(88): p. pe56.

61. Diaz, B., et al., Tks5-dependent, nox-mediated generation of reactive oxygen species is necessary for invadopodia formation. Sci Signal, 2009. 2(88): p. ra53.

62. Mader, C.C., et al., An EGFR-Src-Arg-cortactin pathway mediates functional maturation of invadopodia and breast cancer cell invasion. Cancer Res, 2011. 71(5): p. 1730-41.

63. van Damme, H., et al., The redistribution of cortactin into cell-matrix contact sites in human carcinoma cells with $11 q 13$ amplification is associated with both overexpression and posttranslational modification. J Biol Chem, 1997. 272(11): p. 7374-80.

64. Cobb, B.S., et al., Stable association of pp60src and pp59fyn with the focal adhesion-associated protein tyrosine kinase, pp125FAK. Mol Cell Biol, 1994. 14(1): p. 147-55.

65. Magalhaes, M.A., et al., Cortactin phosphorylation regulates cell invasion through a $p H$ dependent pathway. J Cell Biol, 2011. 195(5): p. 903-20.

66. Charifson, P.S., et al., Peptide ligands of pp60(c-src) SH2 domains: a thermodynamic and structural study. Biochemistry, 1997. 36(21): p. 6283-93.

67. Ang, K.K., et al., Impact of epidermal growth factor receptor expression on survival and pattern of relapse in patients with advanced head and neck carcinoma. Cancer Res, 2002. 62(24): p. 7350-6.

68. Rodrigo, J.P., et al., EMS1 gene amplification correlates with poor prognosis in squamous cell carcinomas of the head and neck. Clin Cancer Res, 2000. 6(8): p. 3177-82.

69. Ammer, A.G., et al., Saracatinib Impairs Head and Neck Squamous Cell Carcinoma Invasion by Disrupting Invadopodia Function. J Cancer Sci Ther, 2009. 1(2): p. 52-61. 
70. Rothschild, B.L., et al., Cortactin overexpression regulates actin-related protein 2/3 complex activity, motility, and invasion in carcinomas with chromosome 11q13 amplification. Cancer Res, 2006. 66(16): p. 8017-25.

71. Shin, N.Y., et al., Subsets of the major tyrosine phosphorylation sites in Crk-associated substrate (CAS) are sufficient to promote cell migration. J Biol Chem, 2004. 279(37): p. 38331-7.

72. Case, D.A., et al., The Amber biomolecular simulation programs. J Comput Chem, 2005. 26(16): p. 1668-88.

73. Homeyer, N., et al., AMBER force-field parameters for phosphorylated amino acids in different protonation states: phosphoserine, phosphothreonine, phosphotyrosine, and phosphohistidine. J Mol Model, 2006. 12(3): p. 281-9. 


\section{Figure Legends}

Figure 1. Src SH2 Binding to Cortactin Does not Involve Tyrosine Phosphorylation and Binds Cortactin Repeats 1 and 5.

(A) GST and GST-Src SH2 affinity precipitation from MTLn3 cells evaluated for cortactin tyrosine phosphorylation. The ratio of phosphorylated cortactin levels and the normalized amounts of total precipitated cortactin are indicated. (B) $\mathrm{Src} \mathrm{SH} 2 \mathrm{Far}$ Western analysis of FLAG-tagged recombinant wild-type and cortactin phosphorylation

mutants. (C) Affinity precipitation analysis of FLAG-tagged recombinant wild-type and cortactin phosphorylation mutants from extracts with GST-Src SH2 domain. (D) Affinity precipitation of non-phosphorylated, recombinant cortactin with GST and GST-Src SH2. Normalized intensity levels are shown relative to GST control. (E) GST-SH2 domain Far Western blotting of the cortactin NTA and repeats region. (F) GST-SH2 Far Western analysis of cortactin deletion cortactin constructs. (G) Far Western binding of the GST-Src SH2 domain to tandem cortactin repeat chimeric constructs. Asterisks indicate position of recombinant cortactin proteins. Arrows denote position of IgG heavy chain $(\mathrm{HC})$ recognized by cross reactivity with secondary antibodies during the blotting process.

Figure 2. Cortactin Cysteines 112 and 246 are Required for Src SH2 Domain Binding

(A) Alignment of cortactin repeats denoting C112 and C246. (B and C) Far Western blotting of GST-Src SH2 domain with cortactin cysteine to alanine mutants. WT; full- 
length wild type cortactin. Dashed line separates heavy chain $(\mathrm{HC})$ from chimeric cortactin proteins (asterisks) due to similar molecular weights. (D) Far Western analysis of $\mathrm{Src} \mathrm{SH} 2$ domain binding to the $\mathrm{C} 112 / \mathrm{C} 246 \mathrm{~A}$ cortactin double cysteine mutant (DCM). (E) Affinity precipitation analysis of $\mathrm{Src} \mathrm{SH} 2$ domain binding to the C112/C246A cortactin double cysteine mutant (DCM).

Figure 3. Cysteine-containing Cortactin Peptides Dock Within the Src SH2 Phosphotyrosine Binding Region

(A) Molecular modelling of the Src SH2 domain with phosphorylated Src and cortactin pentapeptides. Enlarged views show position of Src R175, Src C185 and respective central Src or cortactin peptide residue. (B) Calculated binding energies for each peptide docking condition shown in (A).

Figure 4. Binding and Phosphorylation of Cortactin by Src is Redox Dependent and Requires Src C185

(A) Co-immunoprecipitation of cortactin with Src followed by analysis under reducing (R) and non-reducing (NR) conditions. Asterisks denote equivalent bands in cortactin and Src immunoblots. (B). Co-immunoprecipitation of Src with cortactin followed by analysis under reducing $(R)$ and non-reducing (NR) conditions. Asterisks denote equivalent bands in cortactin and Src immunoblots. (C) Phosphorylation of cortactin by Src in the absence and presence of DTT. (D) Far Western analysis of GST-Src SH2 and C185A. (E) Affinity precipitation analysis of FAK and cortactin binding to GST-SrC SH2 C185A. 


\section{Figure 5. Src C-185 Forms a Cystine Bond with Cortactin C112 and C246}

(A) Sequence of the predicted $\mathrm{Src} \mathrm{SH} 2$ domain tryptic fragment containing C185. Predicted cystine bonding between Src C185 and the cortactin C112 and C246 tryptic peptides with predicted masses are shown below. (B-D) Extracted ion chromatogram

(left) and ion fragmentation spectra (right) from tandem LC-MS/MS of the GST-Src SH2 domain (B), the GST-SH2 domain with the cortactin C112 peptide (C) and the GST-SH2 domain with cortactin C246 peptide (D). Spectra were enlarged to indicate the position of the Src C-185 b-4 ion (boxed in red).

Figure 6. Cortactin C112/246 is Essential for Phosphotyrosine-based Cortactin Function

(A) Analysis of Src-mediated cortactin Y421 phosphorylation in cortactin WT and DCM proteins following reintroduction of Src into SYF cells. Ratio indicates pY421 phosphorylation to total cortactin levels. (B) Analysis of cortactin WT, TYM and DCM tyrosine phosphorylation in MTLn3 cells. Asterisks show position of the 80kDa and 85kDa cortactin forms. (C) Quantitation of ECIS cell adhesion assays $(n=3)$ in control (Ctl) and shRNA (sh) knockdown 1483 cells re-expressing the indicated FLAG-cortactin constructs. (D) Quantitation of ECIS cell migration assays $(n=3)$ in control (Ctl) and shRNA (sh) knockdown 1483 cells re-expressing the indicated FLAG-cortactin constructs. Bars in (C-D) represent residual standard error from one-way ANOVA without the intercept. Asterisks indicate $p$-values that are $\leq 0.001$. (E) OSC19 cells transfected with the indicated FLAG-cortactin constructs were assayed for invadopodia formation and matrix degradation ( $n=50$ cells from three independent experiments). 
Bars represent residual standard error from one-way ANOVA without the intercept. Asterisks indicate $p$-values that are $\leq 0.001$. Arrows indicate Invadopodia and regions of degraded matrix. Scale bar $=20 \mathrm{~mm}$.

\section{Figure 7. Model of cysteine-mediated interactions in cortactin regulation}

(A) Model of cysteine-based cortactin activation and phosphorylation by Src. Phylogenetic co-conservation of cortactin C112/246 and Src C185. Conserved cysteines are in red and the homologous position noted in yellow. (C) Cartoon representation of $\mathrm{Src} \mathrm{SH} 2$ binding to phosphotyrosine and cysteine residues. Src amino acids 172-191 within the $\mathrm{SH} 2$ domain binding pocket are shown in white, interacting arginine 175 and cysteine 185 residues are noted in red. Cystine bonding is indicated as a red line. Phosphotyrosine and cystine binding ligands are listed. (D) Alignment of cysteine-containing $\mathrm{SH} 2$ domains. Domains known to bind ligands in a phosphotyrosine-independent manner are bolded and italicized. Cysteine residues are noted in red. Shaded codes: Green; hydrophobic, blue; + charged, red; - charged, yellow; polar.

Table 1. Observed and predicted ion masses of GST-Src-SH2, GST-Src-SH2 + cortactin C112 and GST-Src-SH2 + cortactin C246 tryptic peptides. 
A

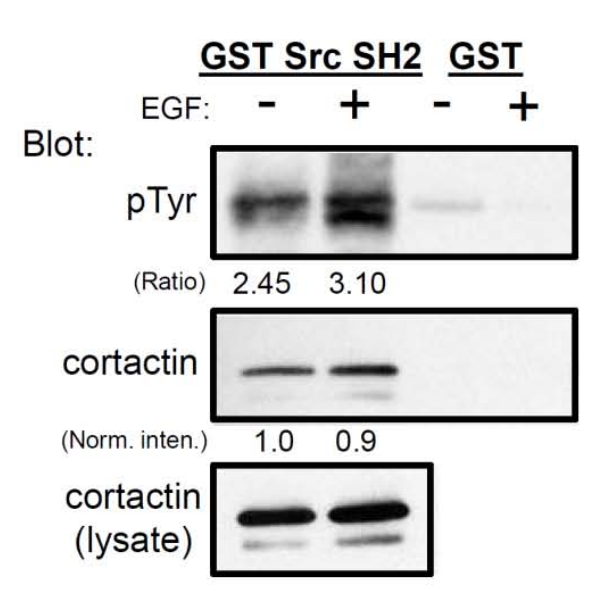

B

Far Western

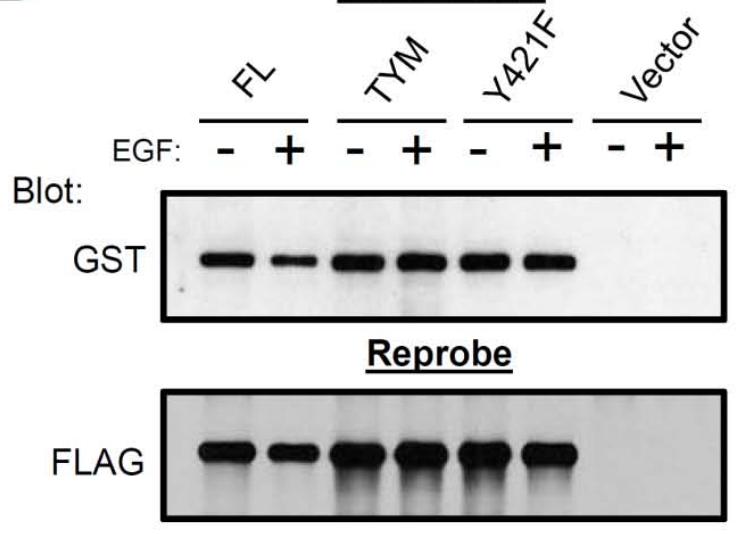

C

GST Src SH2 Lysate

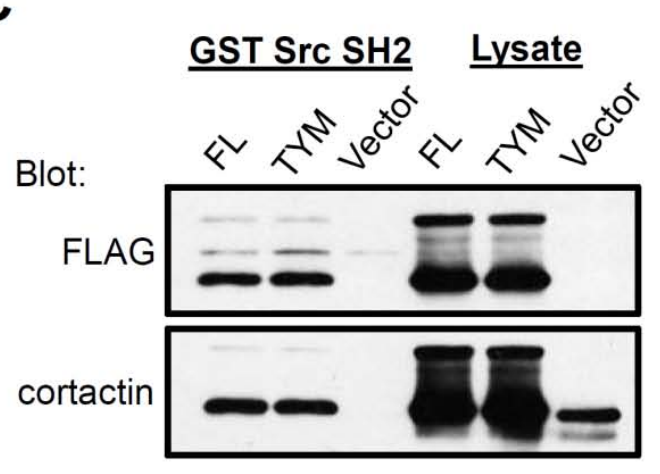

D
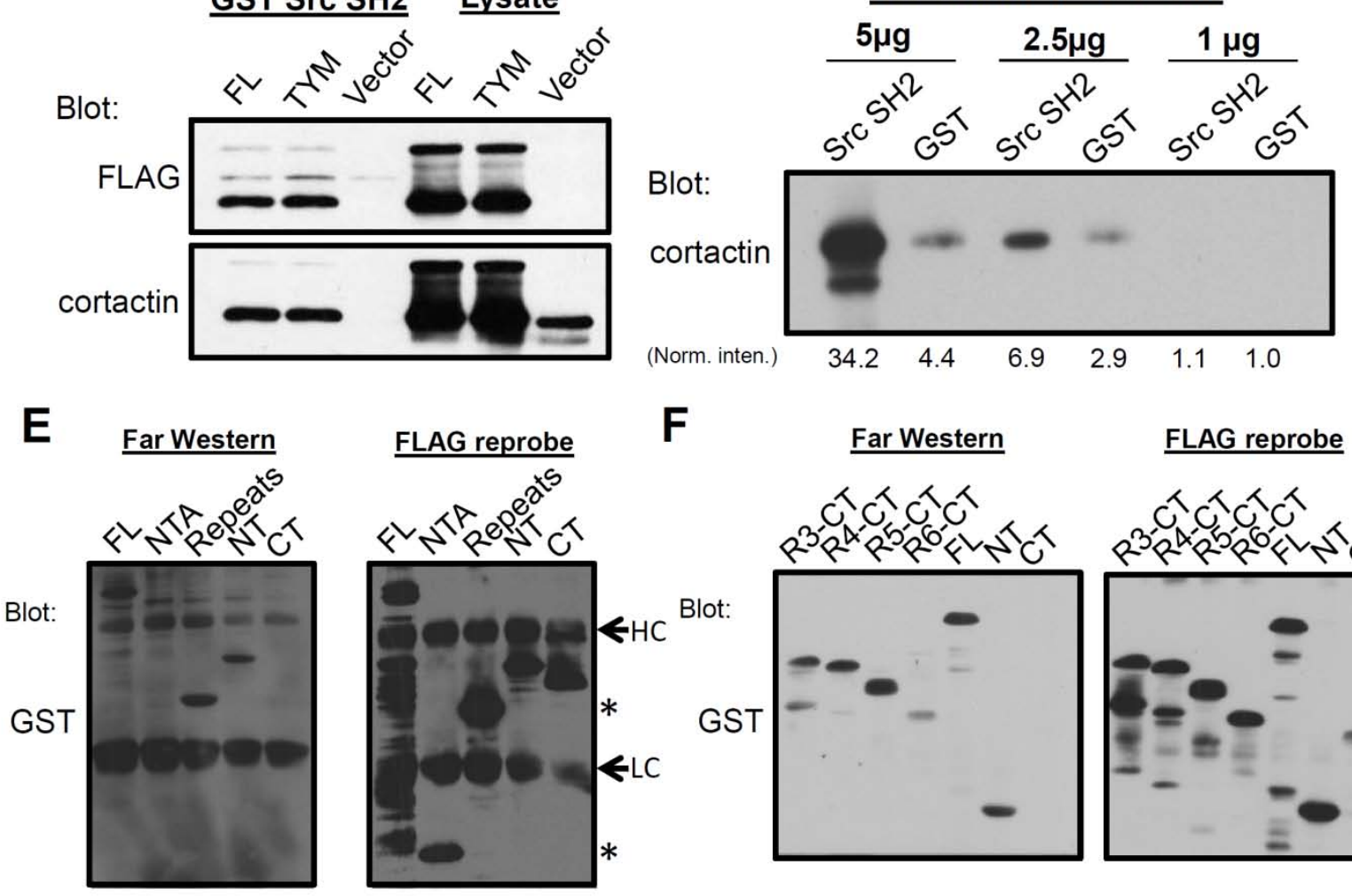

F

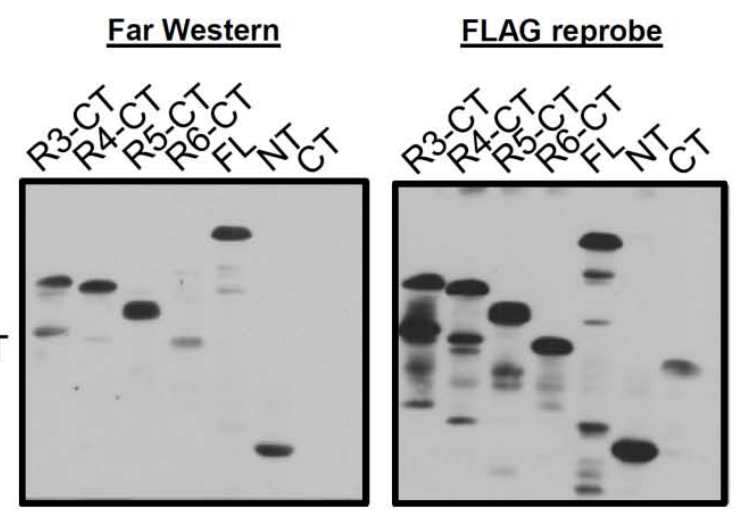

G

Far Western

FLAG reprobe

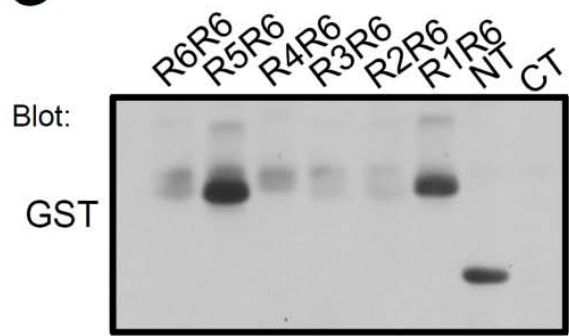

$0^{6} p^{6} R^{6} p^{6} p^{6}, p^{6}$

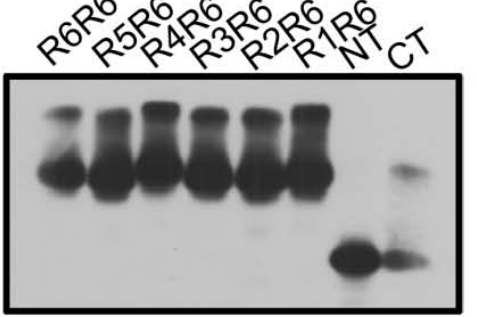

Evans et al. Figure 1 
A

Repeat 1: 85-121 G G K F G V E Q D R M D R S A V G H E Y Q S K L S K HCD Q V D S V R G F Repeat 2: 122-158 G G K F G V Q M D R D Q S A V G F Y Q G K T E K H A S K D Y S G F Repeat 3: 159-195 G G K Y G V Q A D R V D K A V G F Y Q G K T E K H E S K D Y S K F Repeat 4: 196-232 G G K Y G I D K D K V D K S A V G E Y Q G K T E K H E S Q K D Y K G F Repeat 5: 233-269 G G K F G V Q T D R Q D KCA L G W D H E K L Q L H E S Q K D Y K T G F Repeat 6: 270-306 G G K F G V Q S E R Q D S S A V G F D Y K E R L A K H E P Q Q D Y K G F Repeat 7: 307-326

B

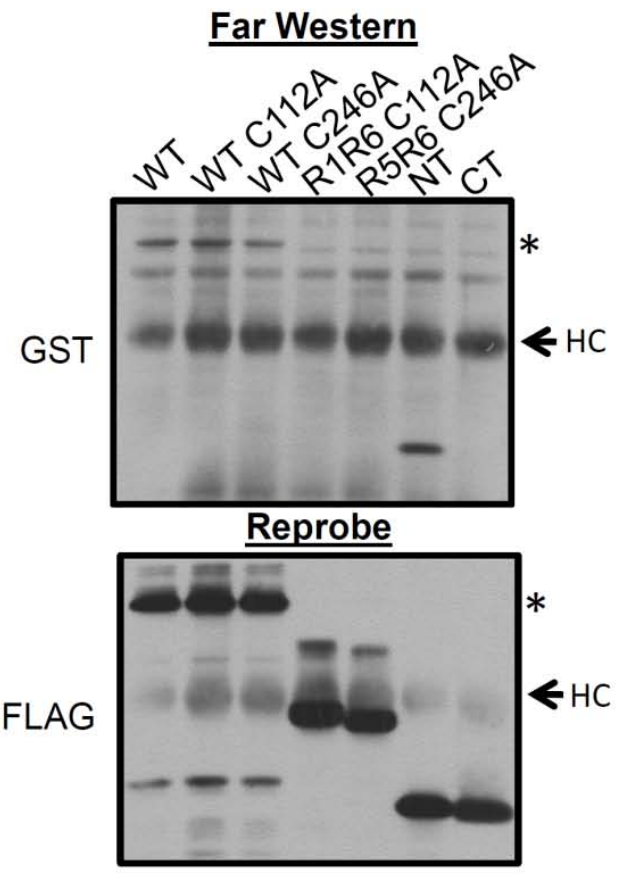

D
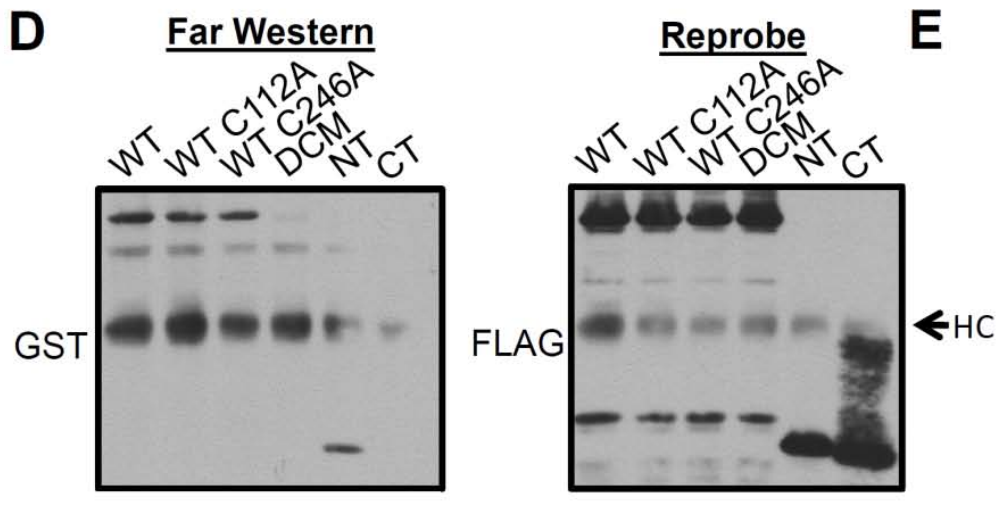

C Far Western

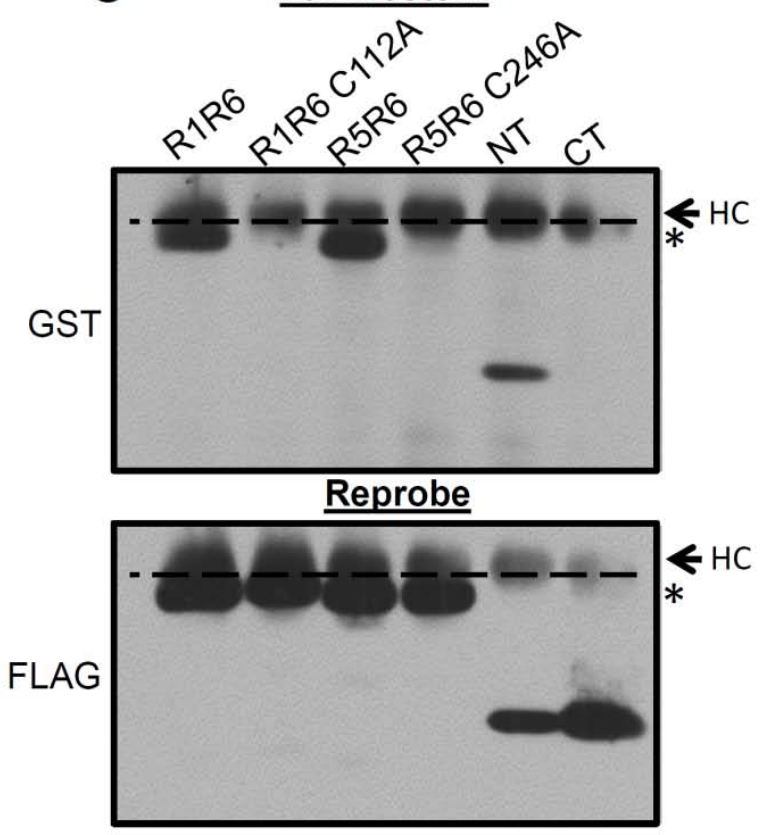

E

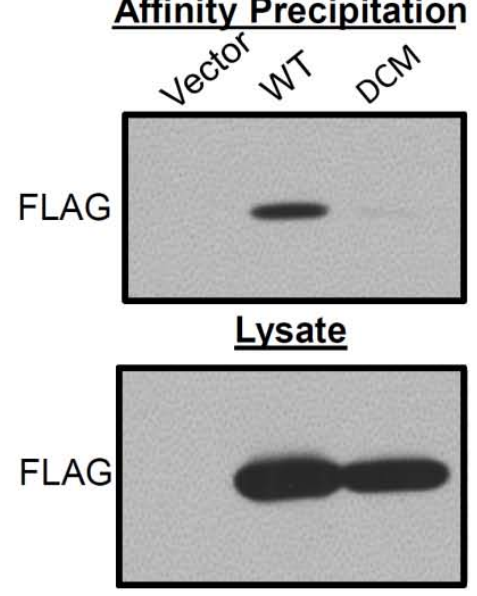

Evans et al. Figure 2 
A

Src SH2

+ pY527

+ C112

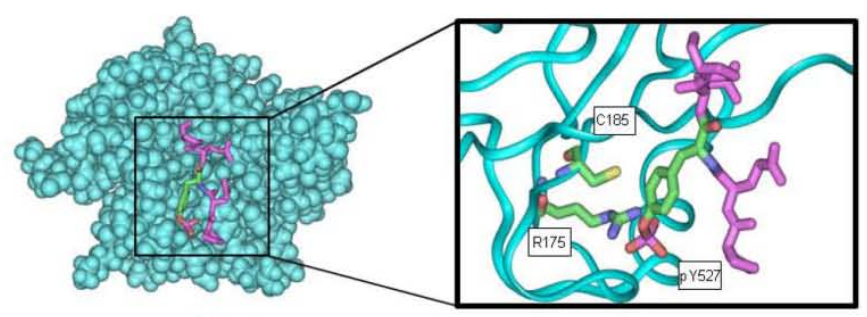

$\mathrm{Src} \mathrm{SH} 2$

$+\mathrm{A} 112$

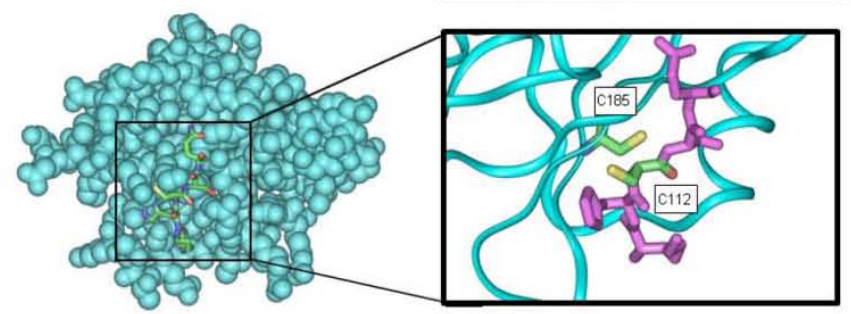

$\mathrm{SrcSH} 2$

$+\mathrm{C} 246$

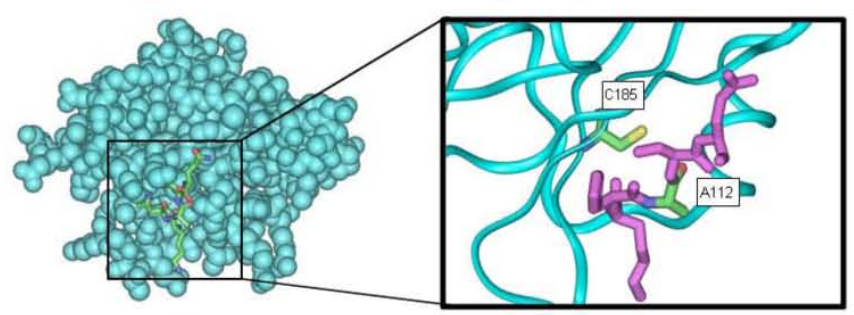

$\mathrm{Src} \mathrm{SH} 2$

$+\mathrm{A} 246$
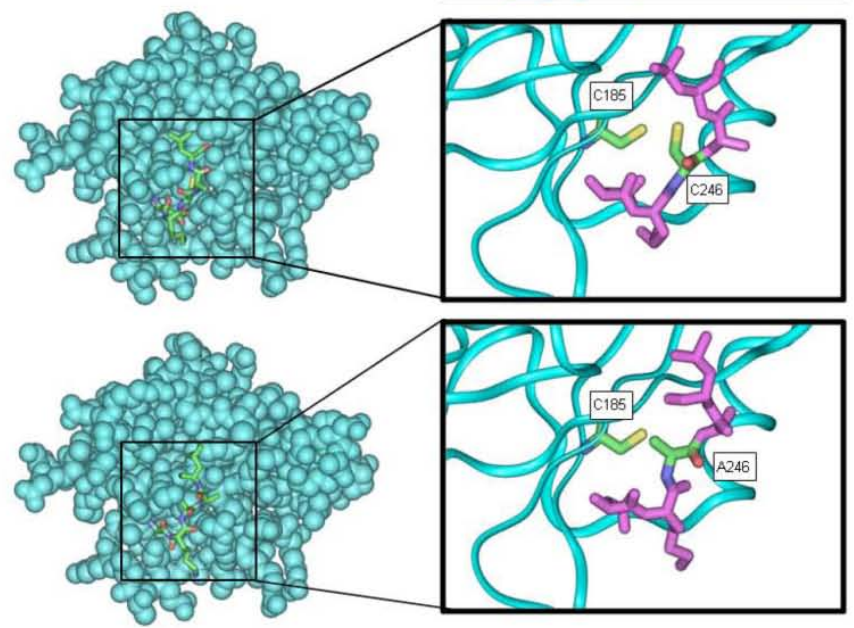

B

\begin{tabular}{|c|c|c|c|c|}
\hline & $\begin{array}{c}\text { Complex } \\
(\mathrm{kcal} / \mathrm{mol})\end{array}$ & $\begin{array}{c}\text { Src SH2 receptor } \\
(\mathrm{kcal} / \mathrm{mol})\end{array}$ & $\begin{array}{c}\text { Src SH2 ligand } \\
(\mathrm{kcal} / \mathrm{mol})\end{array}$ & $\begin{array}{c}\text { Binding Energy } \\
(\mathrm{kcal} / \mathrm{mol})\end{array}$ \\
\hline pY527 & -2486.73 & -2011.70 & -435.40 & -39.63 \\
\hline A112 & -2150.21 & -1962.80 & -172.33 & -15.08 \\
\hline C112 & -2149.22 & $"$ & -164.79 & -21.63 \\
\hline A246 & -2166.48 & $"$ & -187.85 & -15.83 \\
\hline C246 & -2166.17 & $"$ & -180.25 & -23.12 \\
\hline
\end{tabular}

Evans et al. Figure 3 
A Cortactin Blot

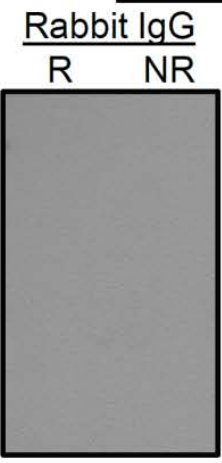

\section{Lysate}

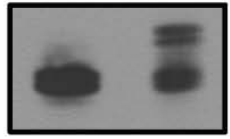

B

\section{Src Blot}

Mouse Ig anti-cortactin IP
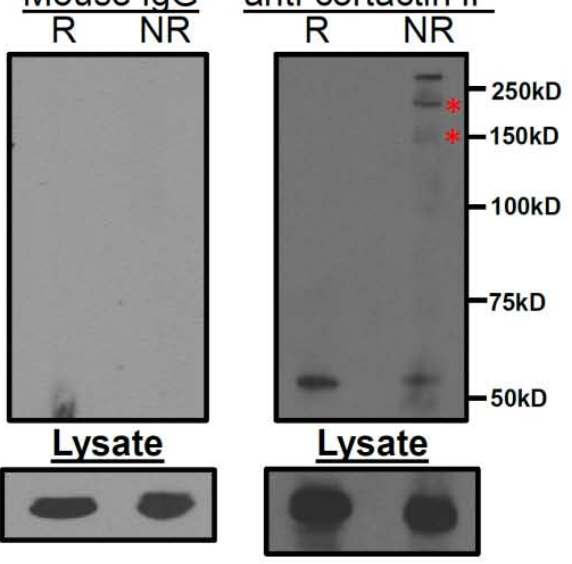

D Far Western (SH2 WT)
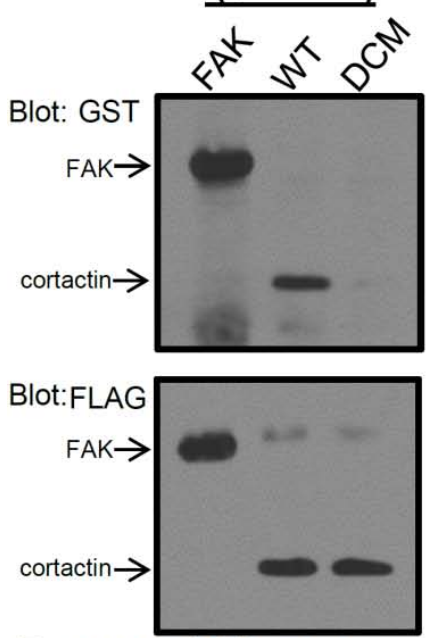

Evans et al. Figure 4
Cortactin Reprobe $\frac{\text { Mouse IgG }}{\mathrm{R}} \frac{\text { anti-cortactin IP }}{\mathrm{NR}}$
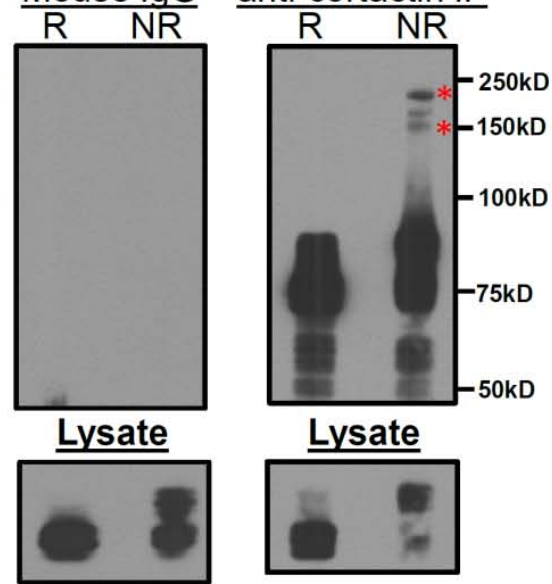

\section{Src Reprobe}

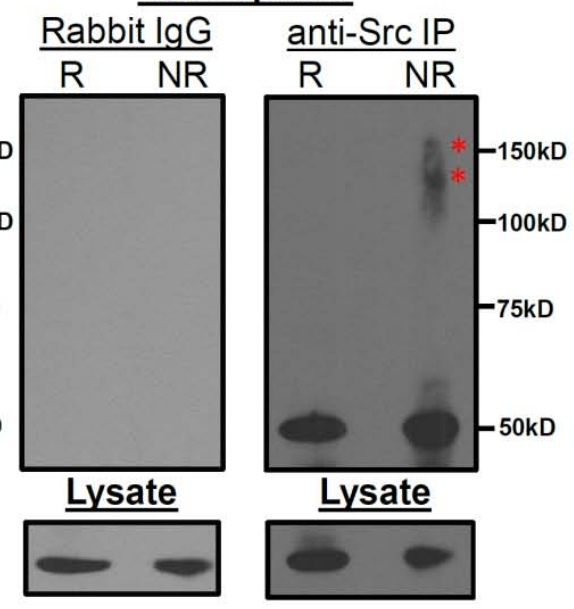

C Src IP Kinase Assay 1mMDTT: - + Blot: pY421
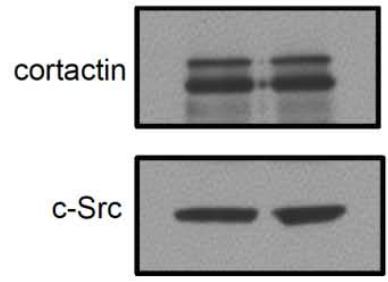

E
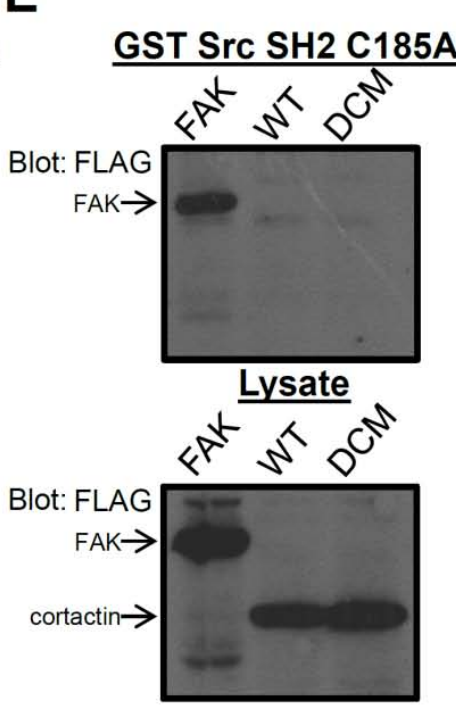
A

Src SH2

Sre Seq: $182 \begin{array}{llllllllllllll}183 & 184 & 185 & 186 & 187 & 188 & 189 & 190 & 191 & 192 & 193 & 194 & 195\end{array}$

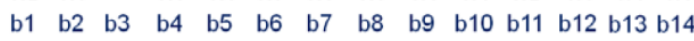
$\underline{\mathrm{G}} \underline{\mathrm{Y}} \underline{\mathrm{C}} \underline{\mathrm{S}} \underline{\mathrm{V}} \underline{\mathrm{S}} \underline{\mathrm{F}} \underline{\mathrm{D}} \underline{\mathrm{A}}$ y14 y13 y12 y11 y10 y9 y8 y7 y6 y5 y4 y3 y2 y1 GST-Src SH2 +

C112 Peptide

GST-Src SH2 +

GAYCLLSVSDFDNAK C246 Peptide

HCSQV $\underset{571.23 \mathrm{Da}}{+}$

GAYCLSVSDFDNAK ] 1488.66 Da

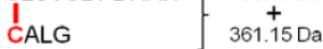

$\overline{2059.89 \mathrm{Da}}$

$\overline{1849.81 \mathrm{Da}}$

B RT: 36.02

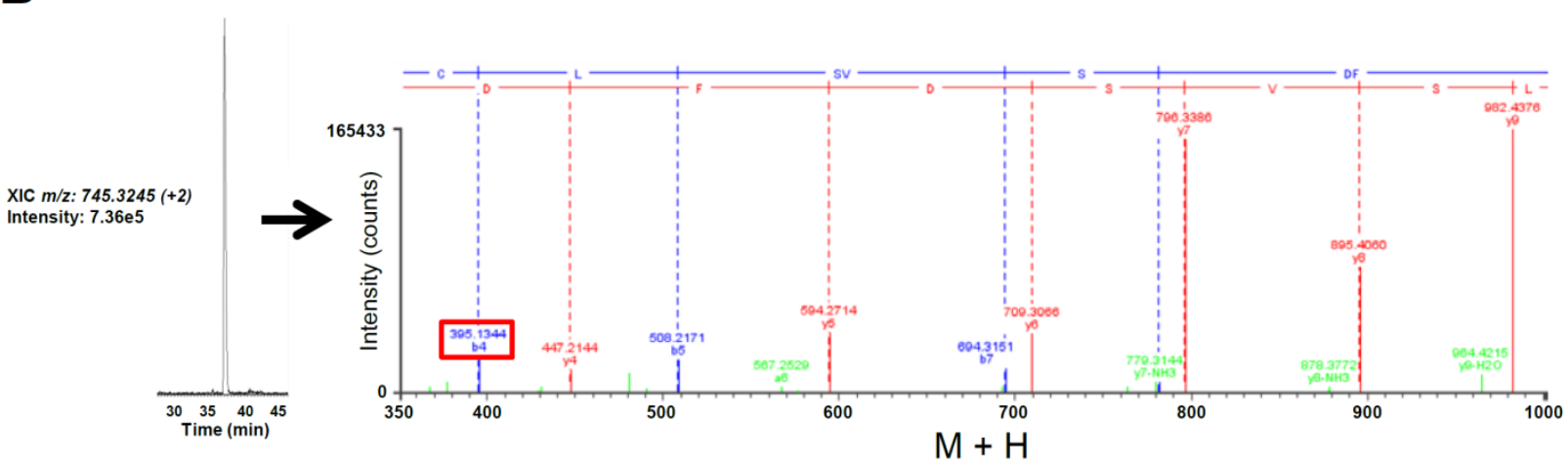

C

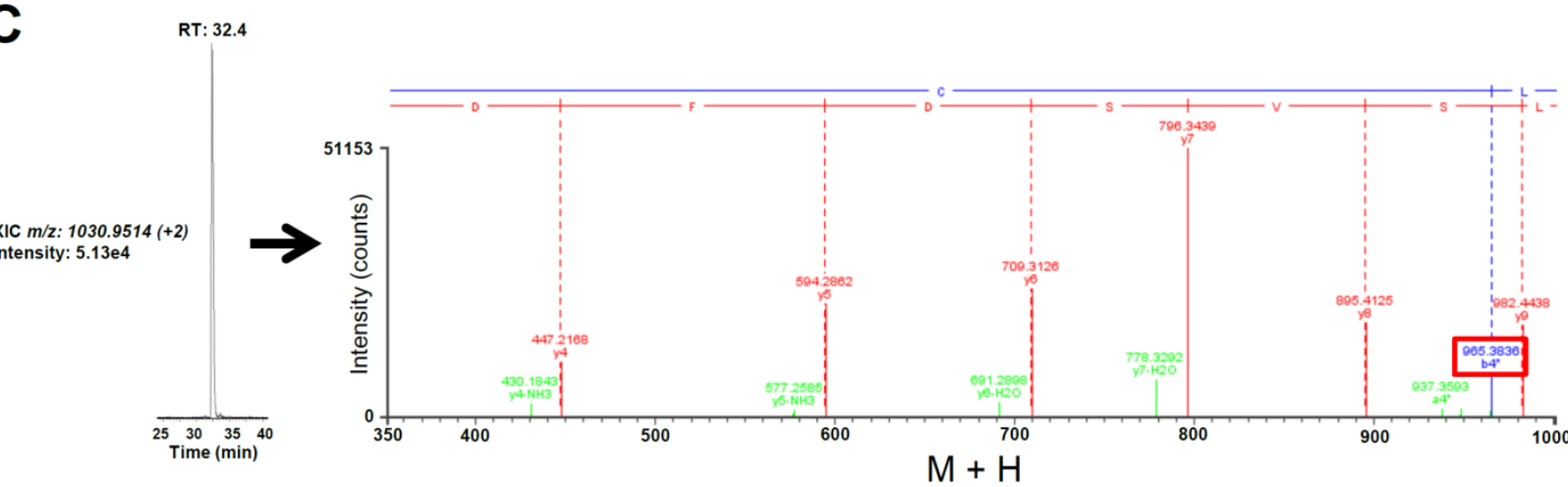

D

RT: 37.92 Intensity: $1.58 \mathrm{e} 5$
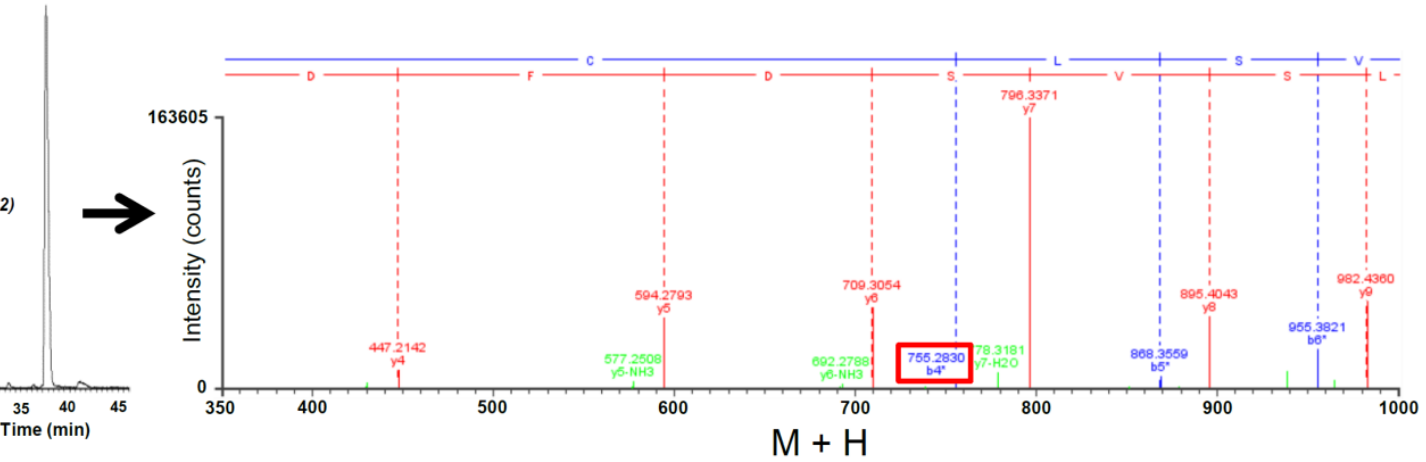

Evans et al. Figure 5 

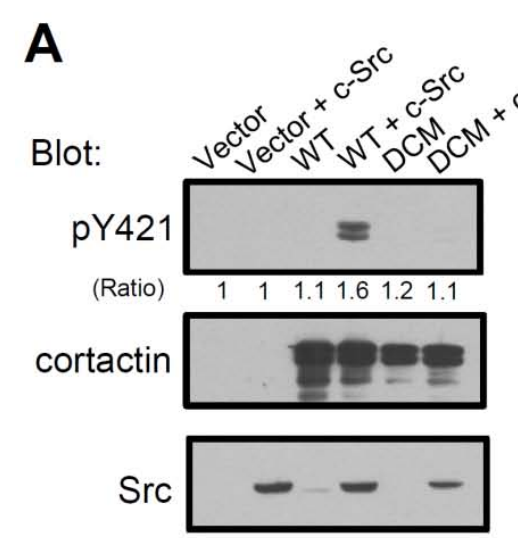

E
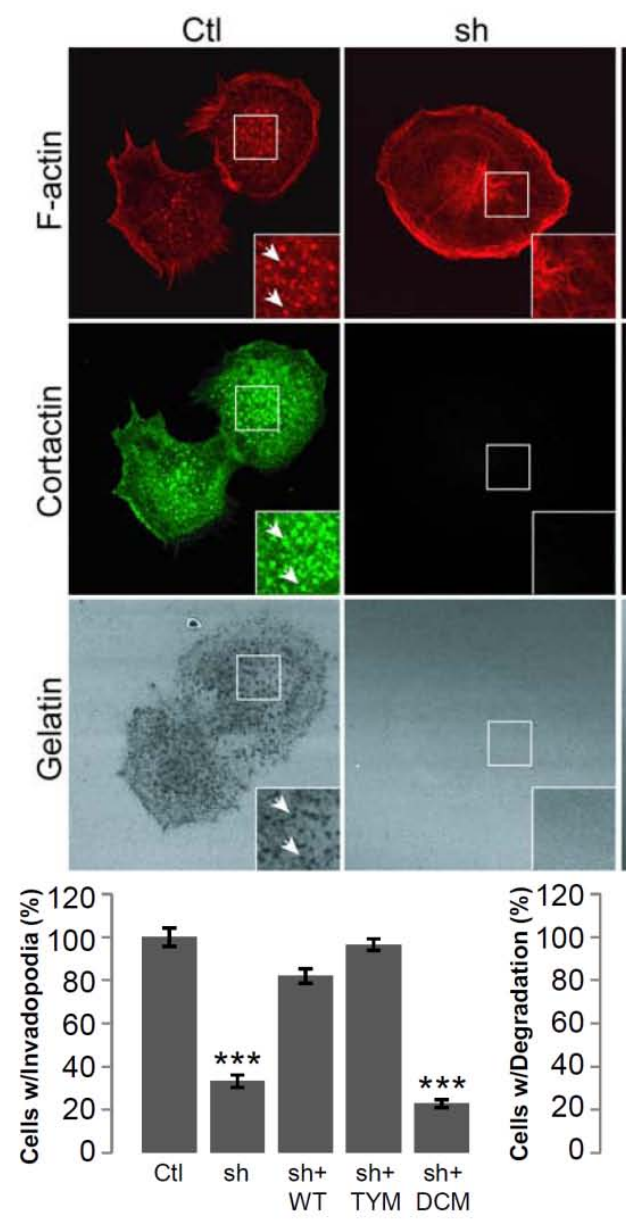

Blot:
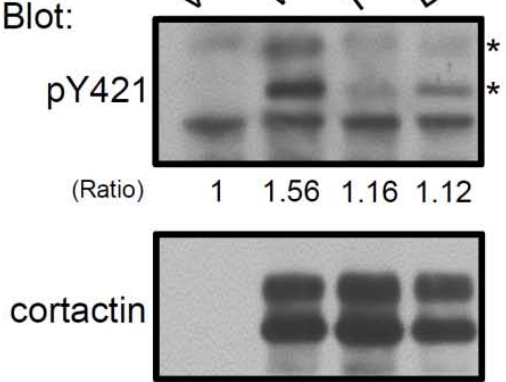

C Cell Adhesion Assay

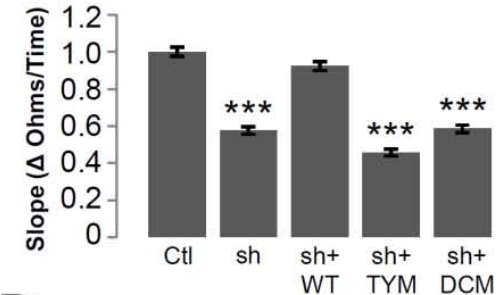

D

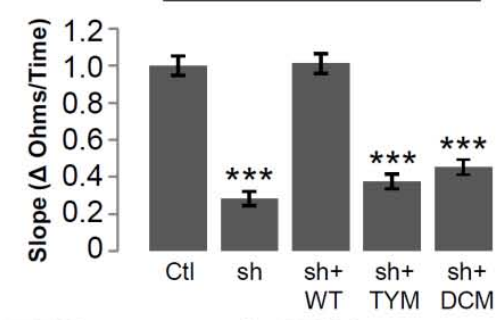

$\mathrm{sh}+\mathrm{WT}$

sh+TYM sh+DCM
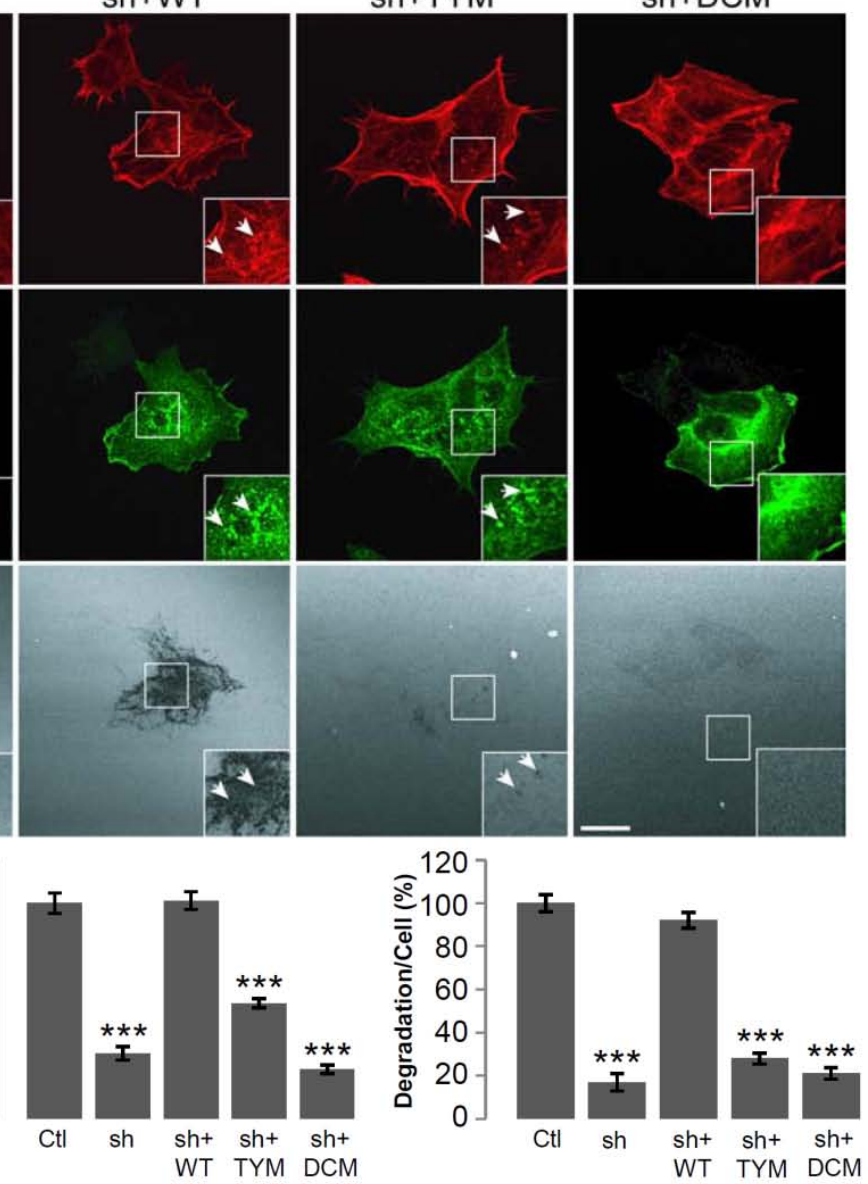

\section{Evans et al. Figure 6}




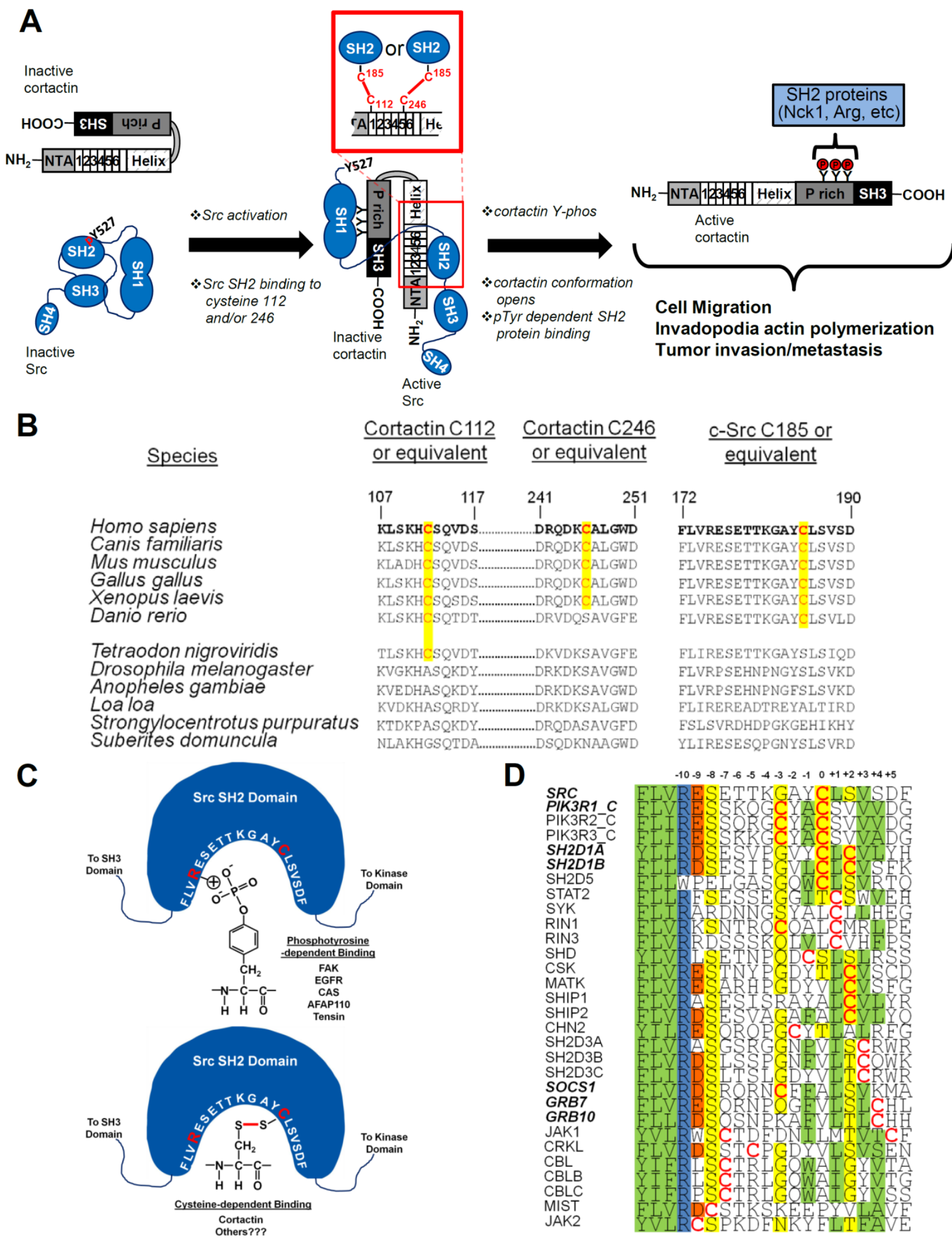

Evans et al. Figure 7 
Table I: LC-MS/MS Analysis of C185 Peptide Modifications

\begin{tabular}{|c|c|c|c|c|c|c|c|c|c|c|c|c|c|c|c|c|c|}
\hline \multicolumn{6}{|c|}{ GST-Src SH2 } & \multicolumn{6}{|c|}{ GST-Src SH2 + C112 Peptide (HCSQV) } & \multicolumn{6}{|c|}{ GST-Src SH2 + C246 Peptide (CALG) } \\
\hline Ion & Exp. & Obs. & Ion & Exp. & Obs. & Ion & $\begin{array}{c}\text { Exp. } \\
\text { da }\end{array}$ & $\begin{array}{c}\text { Obs. } \\
\text { Da. }\end{array}$ & Ion & $\begin{array}{l}\text { Exp. } \\
\text { Da }\end{array}$ & $\begin{array}{c}\text { Obs. } \\
\text { Da. }\end{array}$ & Ion & Exp. & $\begin{array}{l}\text { Obs. } \\
\text { Daa }\end{array}$ & Ion & $\begin{array}{c}\text { Exp. } \\
\text { Da }\end{array}$ & $\begin{array}{c}\text { Obs. } \\
\text { Da }\end{array}$ \\
\hline $\begin{array}{l}\mathrm{b} 1 \\
\mathrm{~b} 2\end{array}$ & $\begin{array}{l}57.02 \\
128.06\end{array}$ & 129.07 & $\begin{array}{l}y 1 \\
y_{2}\end{array}$ & $\begin{array}{l}146.11 \\
217.14\end{array}$ & $\begin{array}{l}1147.11 \\
218.15\end{array}$ & $\begin{array}{l}\mathrm{b} 1 \\
\mathrm{~b} 2\end{array}$ & $\begin{array}{l}57.02 \\
128.06\end{array}$ & 129.07 & $\begin{array}{l}y 1 \\
y 2\end{array}$ & $\begin{array}{l}146.111 \\
217.14\end{array}$ & 218. & $\begin{array}{l}b 1 \\
b 2\end{array}$ & $\begin{array}{l}5.02 \\
128.02\end{array}$ & 129.07 & $\mathrm{y}_{1}$ & $\begin{array}{c}\frac{d a}{146.11} \\
217.14\end{array}$ & $\begin{array}{l}\frac{140}{147.11} \\
218.15\end{array}$ \\
\hline b3 & 291.12 & $\begin{array}{l}202.13 \\
202.13\end{array}$ & y3 & 331.19 & 332.19 & $b_{3}$ & 291.12 & 292.13 & y3 & 331.19 & 332.19 & b3 & 291.12 & $\begin{array}{l}292.13 \\
255.13\end{array}$ & $\begin{array}{c}y=2 \\
y_{3}\end{array}$ & 331.19 & 332.19 \\
\hline b4 & 394.13 & 395.14 & $y^{4}$ & 446.21 & 447.22 & b4 & 965.36 & $965.36^{*}:$ & $y^{4}$ & 446.21 & 447.22 & b4 & 755.29 & $755.28^{\circ}$ & $y_{5}^{4}$ & 446.21 & 447.21 \\
\hline b5 & $\begin{array}{l}507.22 \\
5925\end{array}$ & 508.22 & $y^{5}$ & 593.28 & 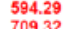 & b5 & 1078.45 & $\begin{array}{l}1078.44^{\circ} \\
1158^{\circ}\end{array}$ & $y 5$ & 593.28 & 594.29 & ${ }^{b 5}$ & 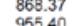 & $\begin{array}{l}868.36^{*} \\
95538^{*}\end{array}$ & $y 5$ & 593.28 & 594.28 \\
\hline $\begin{array}{ll}106 \\
67\end{array}$ & $\begin{array}{l}594.45 \\
693.32\end{array}$ & $69 \overline{69.32}$ & $\begin{array}{l}\text { y } \\
\text { y7 }\end{array}$ & 795.34 & 796.35 & 焉7 & 50.47 & $\begin{array}{l}71664.55^{\circ} \\
1120\end{array}$ & $\begin{array}{l}y_{0} \\
\text { y7 }\end{array}$ & 795.34 & 796.35 & $\begin{array}{l}56 \\
67 \\
67\end{array}$ & $\begin{array}{l}95554.40 \\
1054.47\end{array}$ & $\begin{array}{l}95554.46^{*} \\
1050\end{array}$ & $\begin{array}{l}y_{0}^{16} \\
y^{2}\end{array}$ & $\begin{array}{l}31 \\
34\end{array}$ & $\begin{array}{l}796.31 \\
796.34\end{array}$ \\
\hline $\mathrm{bs}$ & 780.35 & 781.36 & y8 & 894.41 & 895.42 & b8 & 135 & 135 & y8 & 894.41 & 895.42 & $b 8$ & $\begin{array}{l}1144.51 .50 \\
14.5\end{array}$ & $1141.50^{\circ}$ & y8 & 894.41 & 895.40 \\
\hline & 895.38 & & y9 & 981.44 & 982.45 & b9 & 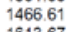 & 146 & (30 & 981.44 & 982.45 & b9 & 1256.53 & $1256.51^{\circ}$ & y9 & 981.44 & 982.44 \\
\hline b10 & 1042.44 & 1043.45 & y10 & 1094.52 & & b 10 & 1613.67 & 161 & y10 & 1094.52 & 1095.53 & b10 & 1403.60 & $1403.58^{*}$ & y10 & 1094.52 & 1095.52 \\
\hline 品1 & $t_{1}^{1}$ & & , & $\begin{array}{l}136060 \\
1360\end{array}$ & & $\begin{array}{l}b 11 \\
b\end{array}$ & 1042 & $1728.70^{\circ}$ & 112 & & $\begin{array}{l}1768.77^{*} \\
1931.83^{*}\end{array}$ & b12 & & $63^{6 *}$ & $\begin{array}{l}y 11 \\
y\end{array}$ & $\begin{array}{l}15 \\
177\end{array}$ & $1558.6^{\circ}$ \\
\hline $\begin{array}{l}\text { D12 } \\
\text { b13 }\end{array}$ & & & y13 & & & 13 & & $1913.78^{\circ}$ & $\begin{array}{l}y 12 \\
y 13\end{array}$ & & $1931.83^{\circ}$ & & & & $\begin{array}{l}\mathrm{y} 12 \\
y^{2}\end{array}$ & & - \\
\hline b14 & 147065 & & y14 & 148866 & 432 & 614 & & & y14 & $\begin{array}{r}2002.80 \\
2059\end{array}$ & 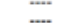 & b14 & & 1003.10 & $\begin{array}{l}113 \\
y 14\end{array}$ & $\begin{array}{l}1792.81 \\
1849\end{array}$ & $=$ \\
\hline
\end{tabular}

Black numerals indicate expected ion masses

Red numerals indicate observed ion masses

* indicates peptide modified masses

Evans et al. Table I 


\section{Supplemental Information}

Figure S1, Related to Figure 1. Src Binding to Cortactin Requires the SH2 Domain and is Phosphotyrosine Indpendent

(A) Affinity precipitation (AP) assays from MTLn3 cells with immobilized GST Src-SH2, $\mathrm{SH} 3$ or tandem $\mathrm{SH} 2-\mathrm{SH} 3$ fusion proteins. Cortactin was detected with mAb 4F11. (B) Cortactin schematic showing protein domains and tyrosine phosphorylation sites. (C) Sequence of cortactin peptides used for $\mathrm{SH} 2$ domain array screening. (D) SH2 domain arrays (Panomics \#MA3040) screened with cortactin peptides.

Figure S2, Related to Figure 1. Phosphorylation of Recombinant Cortactin by Src and Mapping of Src SH2 Binding to the Cortactin Amino Terminal Domain

(A) Phosphorylation of purified recombinant murine cortactin with activated Src monitored by Western blotting with anti-cortactin pY421 antibodies. (B) Far Western blotting of immunoprecipitated FAK proteins with GST-Src SH2 domain. (C) Far Western analysis of cortactin full length (FL), amino terminal (NT) and carboxyl terminal (CT) binding to Src SH2 domain. Far Westerns were probed with anti-GST antibodies to detect bound GST-Src SH2 domain. The position of immunoglobulin heavy chain (HC) is indicated on the right. (D) Affinity precipitation of cortactin proteins with GSTSrc SH2 domain. Co-precipitated and total (lysate) myc-tagged FL, CT and NT cortactin proteins were identified by anti-myc Western blotting.

Figure S3, related to Figure 1. Schematic Diagram of Cortactin Constructs Used in this Study

Deletion and chimeric constructs are shown. NTA; amino terminal acidic domain, R; 
individual cortactin repeat units within the repeats domain, helix; predicted alpha helical domain, $\mathrm{P}$ rich; proline-rich domain, $\mathrm{SH}$, Src homology 3 domain.

Figure S4, related to Figure 1. Src SH2 Domain Does not Bind to the $5^{\text {th }}$ Cortactin Repeat

(A) Far Western binding analysis of the GST-Src SH2 domain with FLAG-WT fulllength cortactin (WT), lacking the $4^{\text {th }}$ cortactin repeat $(\Delta 4)$, lacking the $5^{\text {th }}$ cortactin repeat $(\Delta 5)$, amino terminal $(\mathrm{NT})$ and carboxyl terminal (CT) proteins. (B) Far Western analysis of GST-Src SH2 with the indicated FLAG-cortactin constructs. The membrane was reprobed with an anti-FLAG monoclonal antibody to verify protein expression.

Figure S5. Related to Figure 4. Src SH2 R175A Retains Binding to Cortactin Far Western binding analysis of GST-Src SH2 R175A with full-length (FL),NT and CT cortactin proteins. The membrane was stripped and reprobed with anti-FLAG antibody to verify cortactin fusion protein expression.

Figure S6, Related to Figure 5. Y-ion Analysis of Cystine bonding between Src C185 and cortactin C112 and C246.

(A) LC-MS/MS ion fragmentation spectra of the GST-Src SH2 domain showing the position of $\mathrm{y} 11$. (B) Position of $\mathrm{y} 11$ in the GST-SH2 domain with the cortactin C112 peptide. (C) Position of Y11 in the GST-SH2 domain with cortactin C246 peptide. 
Figure S7, Related to Figure 6. Expression of Cortactin Mutants in Cortactin Knockdown Cells and F-actin Co-sedimentation of Cortactin Mutants

(A) Total cell lysate from 1483 cells (Ctl), cells with stable lentiviral-transduced shRNA cortactin knock-down (sh), and sh cells transfected with FLAG-cortactin WT (WT), TYM, and DCM mutants were analyzed for knockdown efficacy and construct expression by cortactin immunoblotting. (B) Western blot analysis F-actin co-sedimentation from 293T cells transfected with empty vector (EV), cortactin $\mathrm{WT}, \mathrm{DCM}$ and $\Delta 4$ constructs. Constructs in supernatant (S) and pellet (F) fractions were detected with anti-FLAG and percentages of constructs in each fraction determined by densitometry. Quantitation of F-actin co-sedimentation assays. Data are shown from two independent experiments. Bars indicate standard error, asterisk indicate $p$-value $\leq 0.05$. 
A
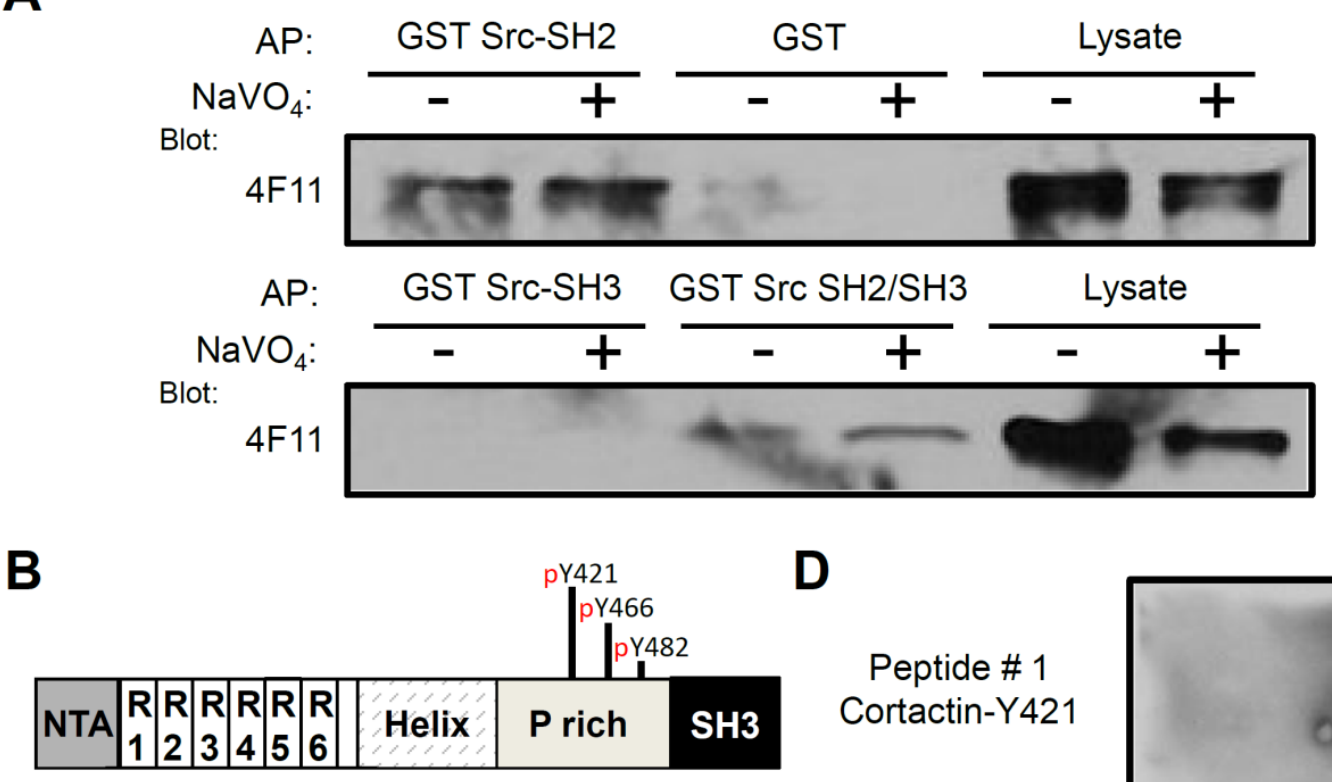

D

Peptide \# 1

Cortactin-Y421

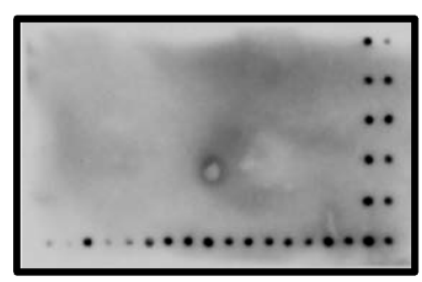

C

1) Cortactin Peptide Y421 Sequence

Biotin-KGGGEDRPPSSPIYEDAAPFKA

2) Cortactin Peptide pY421 Sequence

Biotin-KGGGEDRPPSSPIPYEDAAPFKA

3) Cortactin Peptide Y466 Sequence

Biotin-KGGGLTYTSEPVYETTEAPGH

4) Cortactin Peptide pY466 Sequence

Biotin-KGGGLTYTSEPVPYETTEAPGH

Peptide \# 2

Cortactin-pY421

Src

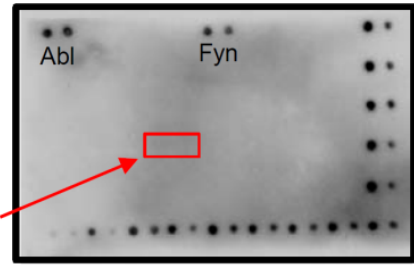

Peptide \# 3

Cortactin-Y466

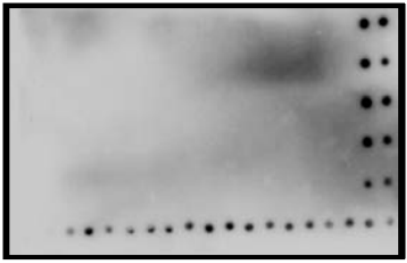

Peptide \# 4

Cortactin-pY466

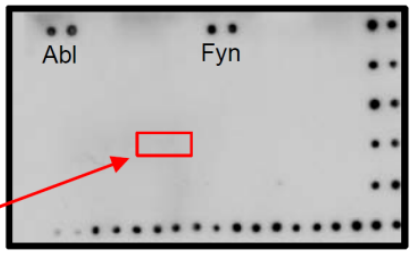

\section{Evans et al. Figure S1}


A

B
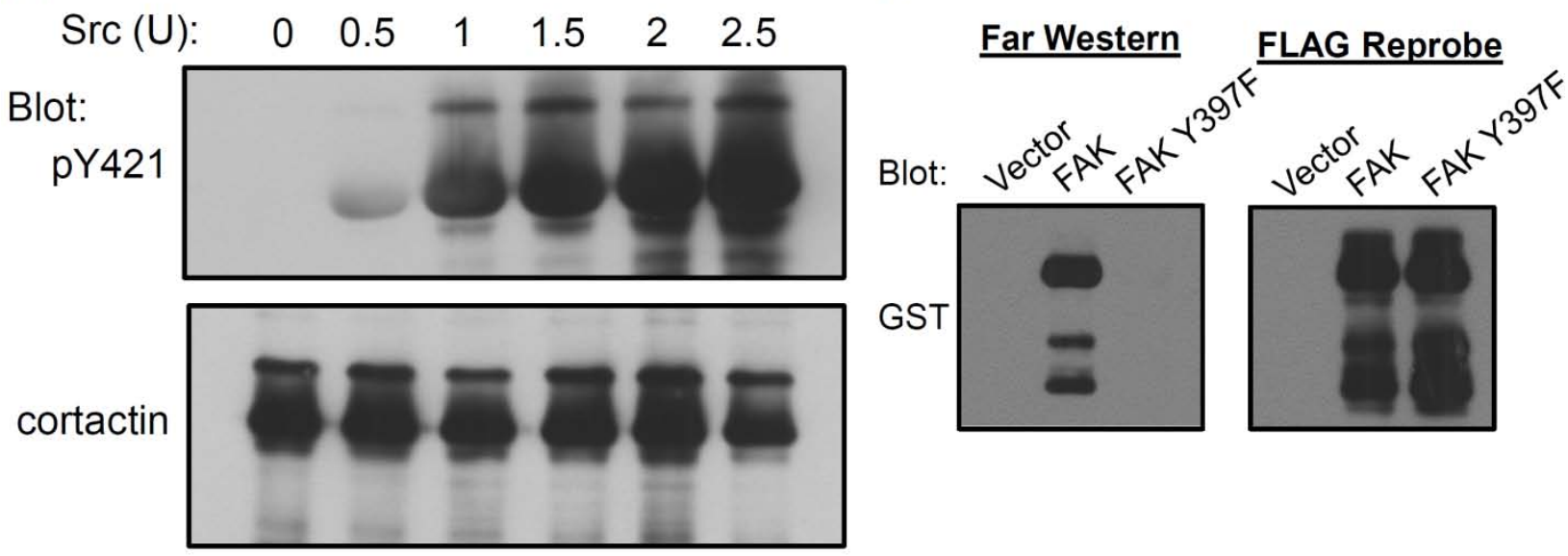

C
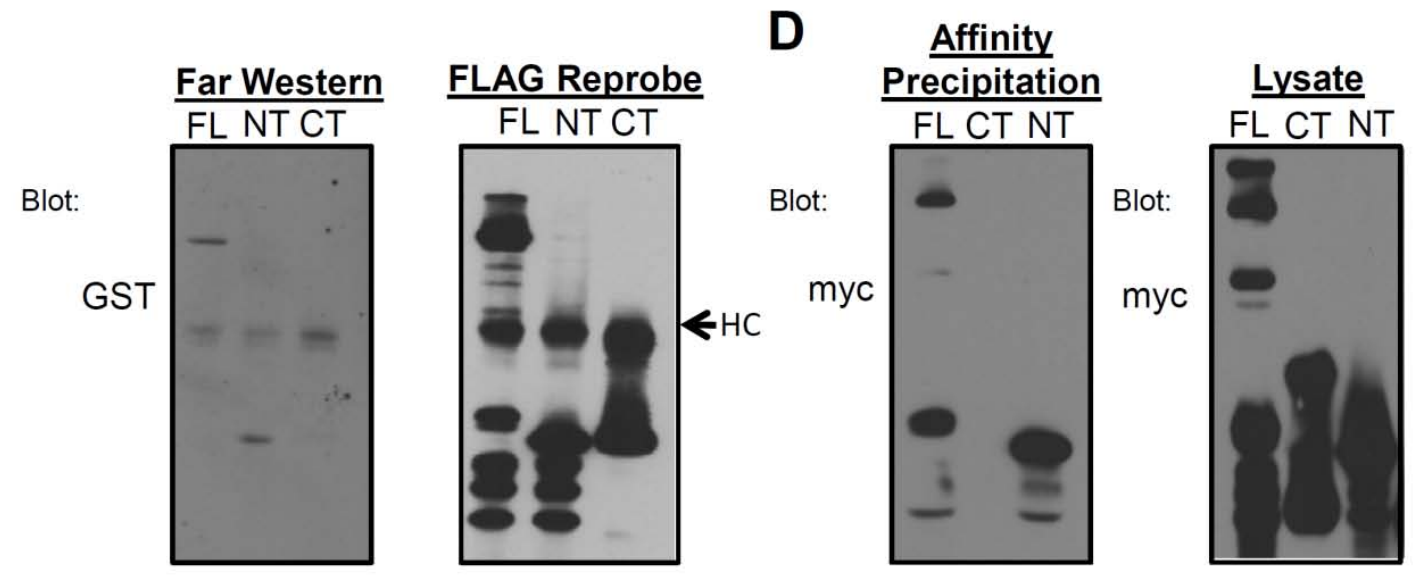

Evans et al. Figure S2 
FL

\begin{tabular}{|c|c|}
\hline NT, & \\
\hline
\end{tabular}

NT

\begin{tabular}{l|l|l|l|l|l|l|} 
NTA & $R$ & $R$ & $R$ & $R$ & $R$ & $R$ \\
& 1 & 2 & 3 & 4 & 5 & 6 \\
\hline
\end{tabular}

CT

NTA

NTA

Repeats

\begin{tabular}{|l|l|l|l|l|l|}
\hline$R$ & $R$ & $R$ & $R$ & $R$ & $R$ \\
1 & 2 & 3 & 4 & 5 & 6 \\
\hline
\end{tabular}

R3-CT

\begin{tabular}{|c|c|c|}
\hline \begin{tabular}{|l|l|l|l}
$\mathrm{R}$ & $\mathrm{R}$ & $\mathrm{R}$ \\
$\mathrm{B}$ & 4 & $\mathrm{R}$ & 6 \\
\end{tabular} & thélí & P rich \\
\hline
\end{tabular}

R4-CT

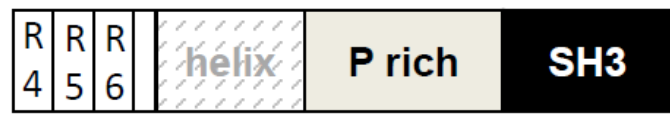

R5-CT

\begin{tabular}{|l|l|l|l|l|}
\hline$R$ & $R$ & $b$ & P rich & SH3 \\
5 & 6 & 6 & \\
\hline
\end{tabular}

R6-CT

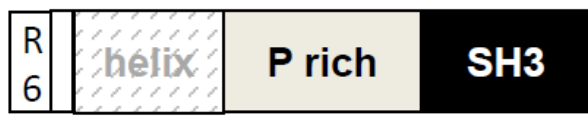

R1/R6-CT

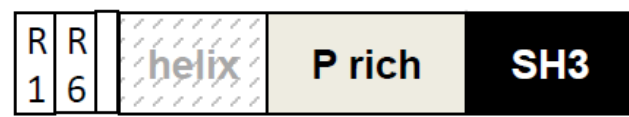

R2/R6-CT

\begin{tabular}{|c|c|c|}
\hline $\begin{array}{l}R \\
R\end{array}$ & éélix & $P$ rich \\
\hline
\end{tabular}

R3/R6-CT

\begin{tabular}{|l|l|l|l|l|}
\hline$R$ & $R$ & & P rich & SH3 \\
3 & 6 & & \\
\hline
\end{tabular}

R4/R6-CT

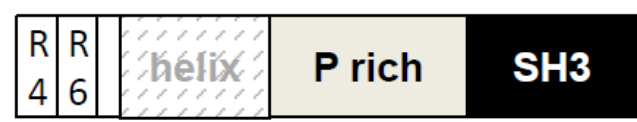

R5/R6-CT

\begin{tabular}{|l|l|l|l|l|}
\hline$R$ & $R$ & \\
5 & 6 & $5 h$ & P rich & SH3 \\
\hline
\end{tabular}

R6/R6-CT

\begin{tabular}{|l|l|l|l|l|}
\hline$R$ & $R$ & & P rich & SH3 \\
\hline & 6 & & \\
\hline
\end{tabular}

Evans et al. Figure S3 
A

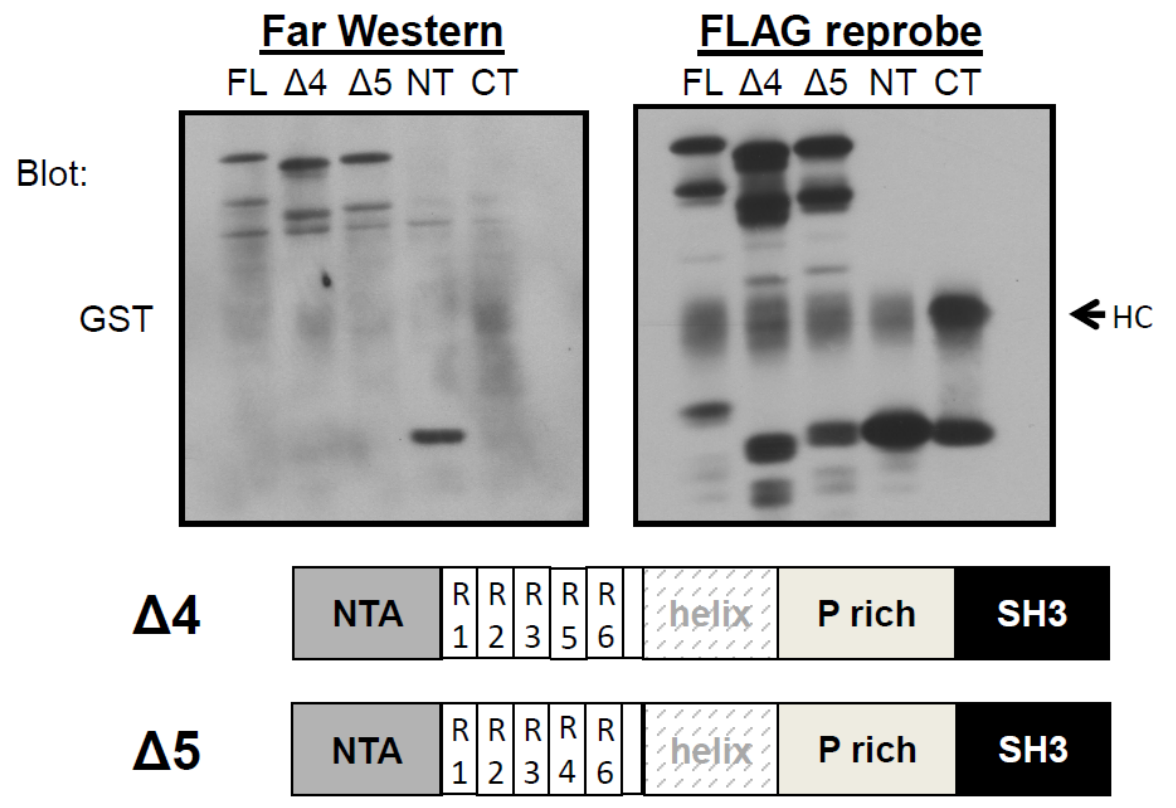

B

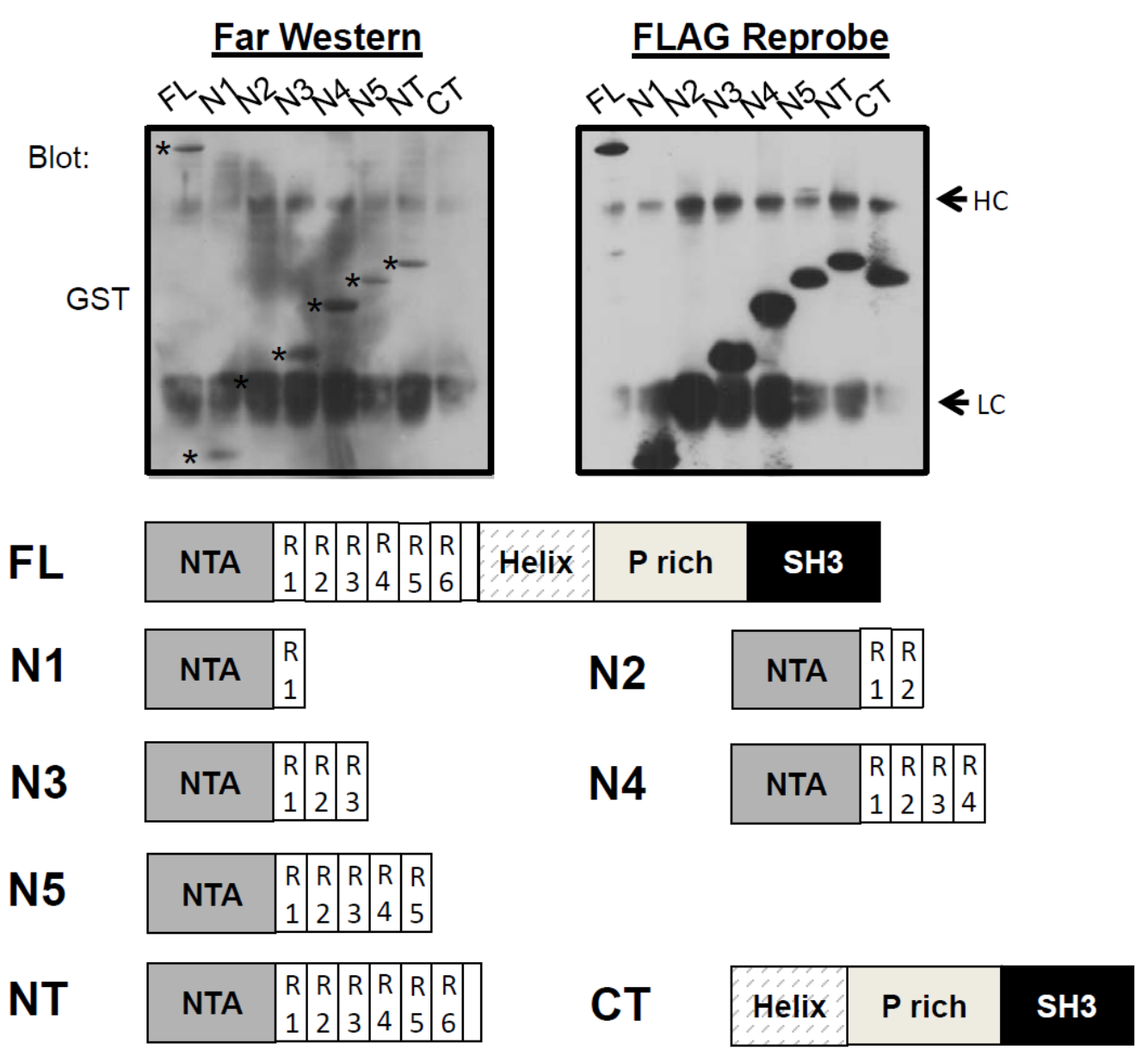

Evans et al. Figure S4 


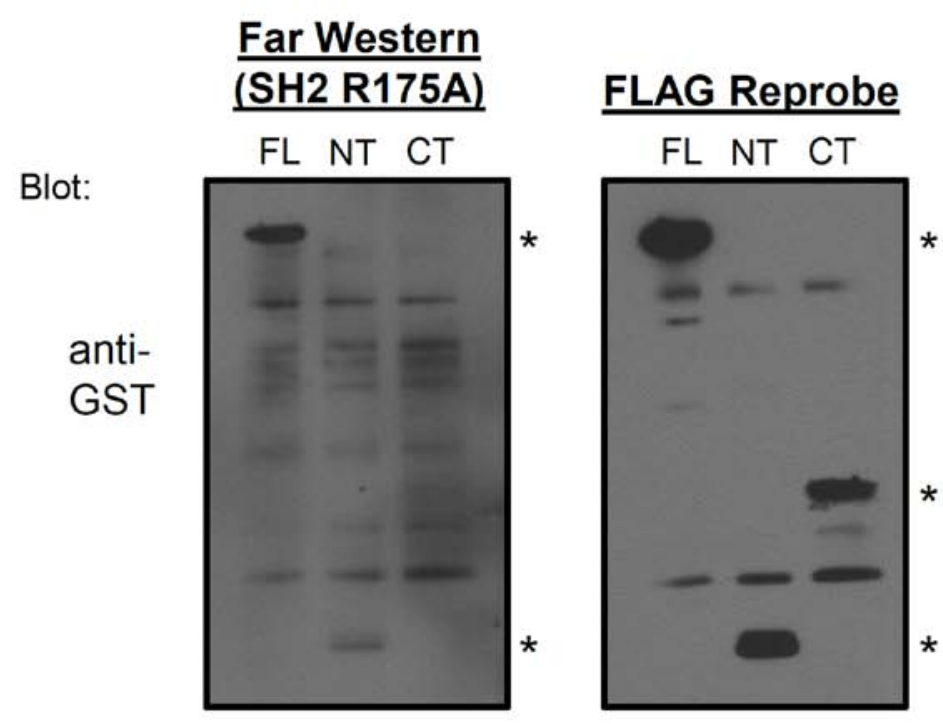

Evans et al. Figure S5 
A

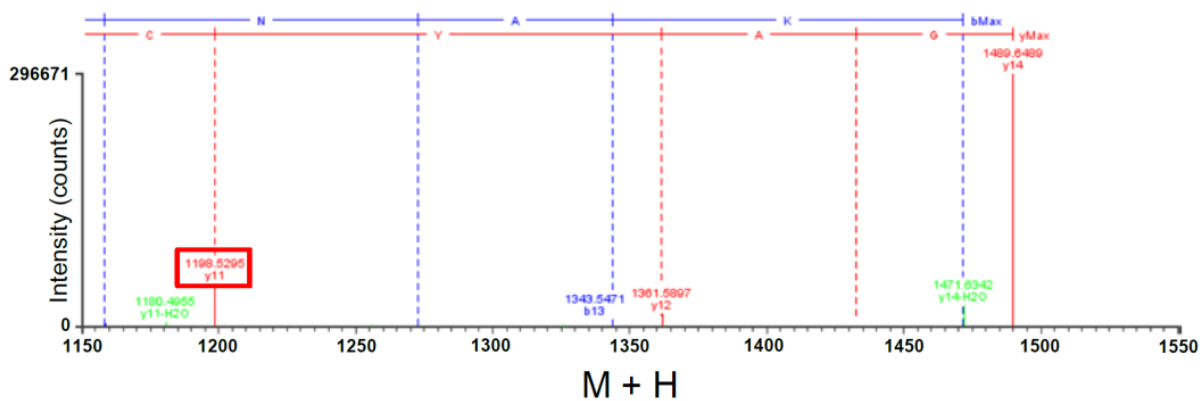

B

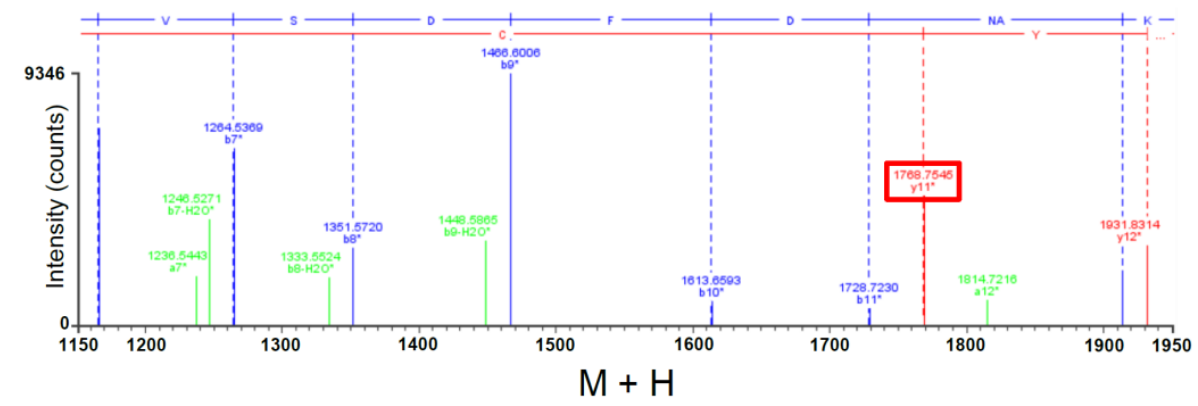

C

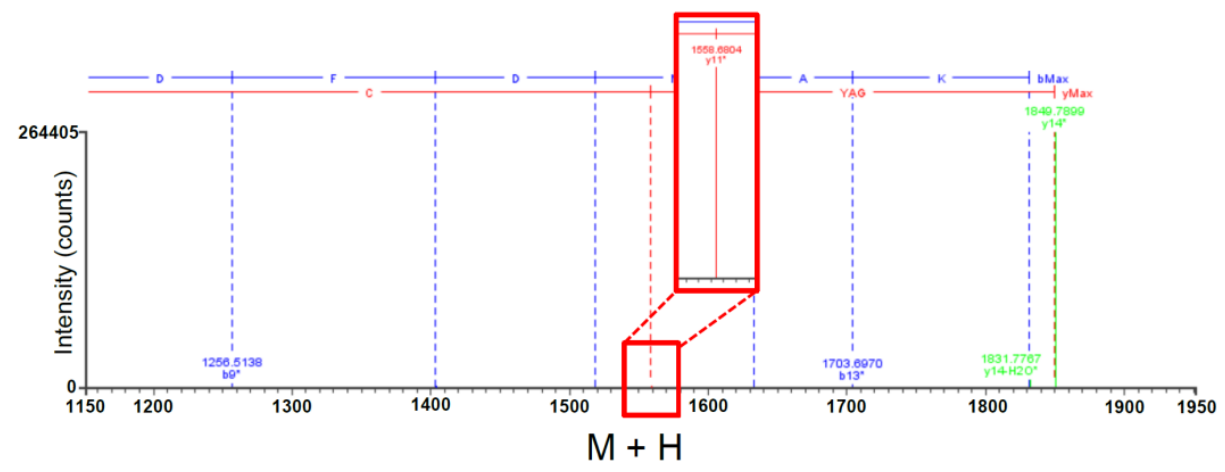

Evans et al. Figure S6 
A

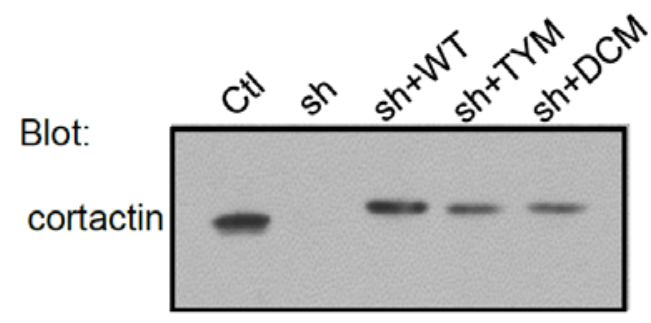

B

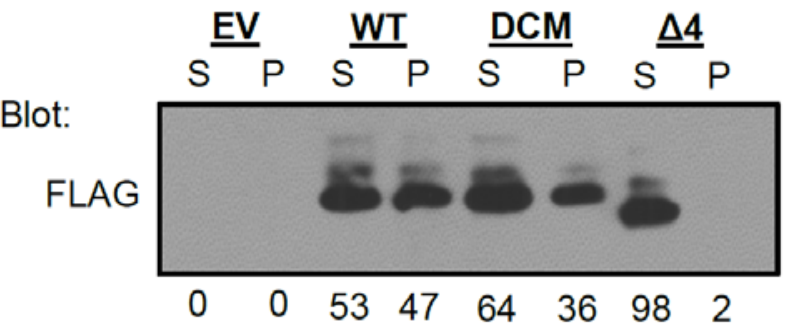

C

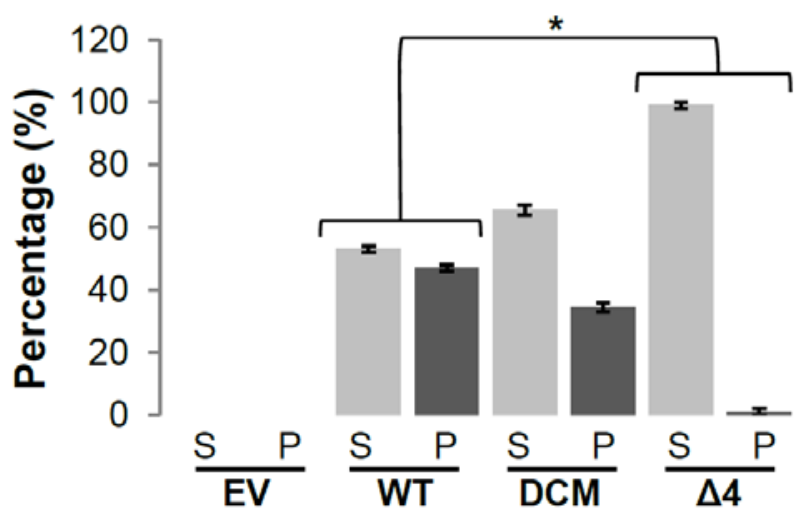

Evans et al. Figure S7 


\section{Study 2: Further Insights into Cortactin Conformational Regulation}

Jason V. Evans, Laura C. Kelley, Karen E. Hayes, Amanda Gatesman Ammer, Karen H. Martin and Scott A. Weed*

Department of Neurobiology and Anatomy, Program in Cancer Cell Biology, Mary Babb Randolph Cancer Center, West Virginia University, Morgantown, WV, USA

${ }^{*}$ Correspondence to: Scott A. Weed; Email: sweed@hsc.wvu.edu

Key words: cortactin, conformation, ERK1/2, Src, N-WASp, phosphorylation, circular dichroism, motility, invasion

Addendum to: Kelley LC, Hayes KE, Ammer AG, Martin KH, Weed SA. Cortactin phosphorylated by ERK1/2 localizes to sites of dynamic actin regulation and is required for carcinoma lamellipodia persistence. PLoS ONE 2010; 5(11):e13847; PMID: 21079800 


\begin{abstract}
The actin regulatory protein cortactin is involved in multiple signaling pathways impinging on the cortical actin cytoskeleton. Cortactin is phosphorylated by ERK1/2 and Src family tyrosine kinases, resulting in neuronal Wiskott Aldrich Syndrome protein (NWASp) activation and enhanced actin related protein (Arp)2/3-mediated actin nucleation. Cortactin migrates as an $80 / 85 \mathrm{kDa}$ doublet when analyzed by SDS-PAGE. Phosphorylation by ERK1/2 is associated with conversion of the $80 \mathrm{kDa}$ to the $85 \mathrm{kDa}$ form, postulated to occur by inducing a conformational alteration that releases the carboxyl-terminal SH3 domain from autoinhibition. Our recent analysis of the $80-85 \mathrm{kDa}$ cortactin "shift" in tumor cells indicates that while ERK1/2 phosphorylation is associated with the $85 \mathrm{kDa}$ shift, this phosphorylation event is not required for the shift to occur, nor does ERK1/2 phosphorylation appreciably alter global cortactin confirmation. These data indicate that additional factors besides ERK1/2 phosphorylation contribute to generating and/or maintaining the activated $85 \mathrm{kDa}$ cortactin form in stimulated cells.
\end{abstract}


Changes in protein conformation are important for generating and propagating intracellular signal transduction events. During normal and pathogenic cell motility, regulation of actin cytoskeletal dynamics responsible for generating movement is dependent on conformational alterations in protein relay systems that activate and terminate signaling pathways responsible for initiating and maintaining motility. Proteins at the intersection of this molecular circuitry are key mediators in motility-driven signal regulation. The filamentous (F)-actin binding protein cortactin interacts with Arp2/3 complex to stimulate and stabilize Arp2/3-F-actin networks in lamellipodia and invadopodia of motile and invasive tumor cells.[1, 2] Cortactin is also a substrate for multiple protein kinases; most notably by ERK1/2 at S405 and S418[3], and Src family tyrosine kinases at Y421, Y470 and Y486.[2] The ERK1/2 and Src phosphorylation sites all reside within a proline-rich region adjacent to a SH3 domain at the extreme carboxyl terminus[2, 3]. Phosphorylation of cortactin by ERK1/2 and tyrosine kinases promotes tumor cell migration and is required for invadopodia-mediated extracellular matrix degradation activity[4, 5], demonstrating important functional roles for these phosphorylation events in cancer progression.

At the mechanistic level, cortactin tyrosine phosphorylation stimulates pro-invasive activity by providing binding sites at $\mathrm{Y} 421$ and $\mathrm{Y} 466$ for the $\mathrm{SH} 2$ domain of the adaptor protein NCK1.[6-8] NCK1, through its SH3 domain, binds N-WASp to release an acidic carboxyl-terminal (VCA) domain that is responsible for binding to and activating Arp2/3 complex, promoting actin polymerization. $[9,10]$ In the case of ERK1/2, cortactin phosphorylation at S405/418 promotes association of the carboxyl-terminal SH3 domain with N-WASp, resulting in a similar Arp2/3 activation cascade.[11] 
Cortactin purified from most cell types as well as from recombinant cell-free sources typically migrates as an 80/85kDa doublet in SDS-PAGE gels.[12-14] This doublet most likely represents a single polypeptide, since electrophoresis in urea-containing gels results in the sole presence of the $85 \mathrm{kDa}$ form[14], supporting the notion that the $80 \mathrm{kDa}$ and $85 \mathrm{kDa}$ cortactin bands represent different conformational isomers. Phosphorylation of cortactin downstream of epidermal growth factor receptor (EGFR) activation results in a shift from $80 \mathrm{kDa}$ to $85 \mathrm{kDa}$. Under these conditions the $85 \mathrm{kDa}$ cortactin form displays increased serine and threonine phosphorylation[15], with the "shift" in cortactin $M_{r}$ occurring concurrently with ERK1/2-mediated S405 and S418 phosphorylation.[3] Collectively these reports have resulted in proposing that non-phosphorylated cortactin exists in the $80 \mathrm{kDa}$ "closed" form with the carboxyl-terminal SH3 domain binding back upon the proline-rich cortactin domain, blocking the ability of the $\mathrm{SH} 3$ domain binding interface to interact with other ligands. ERK1/2 phosphorylation in turn results in displacing the SH3-proline-rich homotypic cortactin interaction, rendering cortactin in an "open" 85kDa state where the SH3 domain can bind N-WASp and other cortactin SH3 binding proteins. $[3,11,16]$ These conclusions are supported by chemical crosslinking studies with non-phosphorylated cortactin that indicate cortactin exists as a monomeric globular protein in solution, with the SH3 domain in close proximity to a helical domain amino terminal to the proline-rich region[17] as well as the prevalence of the $85 \mathrm{kDa}$ form in metastatic colorectal carcinoma cases.[18]

We recently analyzed the cortactin "shift" through a combination of phosphorylationspecific antibodies against cortactin pS405/pS418 and point mutant constructs for these 
residues. Phosphorylation-specific antibodies confirm the presence of pS405 and pS418 predominantly in the $85 \mathrm{kDa}$ cortactin form in tumor cells following EGF treatment. However, analysis of the cortactin shift with phosphorylation-null point mutants downstream of $\mathrm{v}$-Src mediated ERK1/2 activation reveals a more intricate result. Cortactin S418A and S405A/S418A proteins exhibit the same electrophoretic mobility as wild-type cortactin, with the prominent band at $80 \mathrm{kDa}$, while a S405A cortactin mutant runs at the shifted $85 \mathrm{kDa} M_{\mathrm{r}}$. This could suggest that S418 phosphorylation alone is responsible for driving the $80 / 85 \mathrm{kDa}$ cortactin shift, with S405 phosphorylation occurring subsequent to $\mathrm{S} 418$ phosphorylation in the $85 \mathrm{kDa}$ form. However, cortactin phosphorylated by ERK1 at pS418 in vitro does not shift from $80 \mathrm{kDa}$ to $85 \mathrm{kDa}$ (Fig. 1A) and ERK1-phosphorylated cortactin does not demonstrate significant alterations in secondary structure compared to non-phosphorylated cortactin when evaluated by circular dichroism (Fig. 1B). These results demonstrate that ERKmediated cortactin phosphorylation on S405 and S418 is associated with, but is not exclusively responsible for production of the $85 \mathrm{kDa}$ open cortactin form.

We conclude that other factors besides S405/418 phosphorylation are involved in generating and/or maintaining the cortactin shift in EGF-stimulated cells. While the identity of these additional regulatory elements is presently unclear, the proline- rich region where serine 405 and serine 418 reside allows ample opportunity for substantial global conformational alterations through cis-trans isomerization of proline peptide bonds by prolyl isomerases.[19] The compact globular conformation (presumably representative of the closed $80 \mathrm{kDa}$ isomer), with the $\mathrm{SH} 3$ domain folding back onto amino-terminal peptide sequences assumed by cortactin in solution[17] is in contrast to 
the $220 \AA$-long rod-like cortactin protein characterized by rotary shadowing and electron microscopy[20] that may represent the $85 \mathrm{kDa}$ form. These studies provide indirect evidence for involvement of the proline-rich region in extensively altering cortactin structure. 


\section{Acknowledgements}

We thank P. Gannett and J. Jett for assistance with circular dichroism work and data analysis. This work was supported by a subproject of NIH grant P20-RR16440 to SAW. 


\section{Figure Legends}

Figure 1. Analysis of cortactin secondary structure following ERK1 phosphorylation. (A) Phosphorylation of cortactin by ERK1. Recombinant cortactin $(25 \mu \mathrm{g})$ was phosphorylated with the indicated amounts of ERK1 at $30^{\circ} \mathrm{C}$ for $30 \mathrm{~min}$. Aliquots of each reaction were analyzed by Western blotting with anti-pS418 and total cortactin antibodies. The positions of the 80kDa and $85 \mathrm{kDa}$ forms are denoted. (B) Kinase reactions containing recombinant cortactin $(25 \mu \mathrm{g})$ and ATP $(500 \mu \mathrm{M})$ incubated without (red line) or with (blue line) 250ng ERK1 were analyzed with a Jasco J-810 Spectropolarimeter using a scan speed of 50nm/min with a 4 sec response time, 1 $\mathrm{nm}$ band width and $0.1 \mathrm{~nm}$ data pitch (top). The absorbance for each reaction was monitored throughout the analyzed wavelength range (bottom) to control for sample-to-sample variability. Scans are shown for each reaction condition following rendering with Spectra Manager software ( $v$ 1.53.01). 


\section{References}

1. Weaver, A.M., Cortactin in tumor invasiveness. Cancer Lett, 2008. 265(2): p. $157-66$.

2. Ammer, A.G. and S.A. Weed, Cortactin branches out: roles in regulating protrusive actin dynamics. Cell Motil Cytoskeleton, 2008. 65(9): p. 687-707.

3. Campbell, D.H., R.L. Sutherland, and R.J. Daly, Signaling pathways and structural domains required for phosphorylation of EMS1/cortactin. Cancer Res, 1999. 59(20): p. 5376-85.

4. Kruchten, A.E., et al., Distinct phospho-forms of cortactin differentially regulate actin polymerization and focal adhesions. Am J Physiol Cell Physiol, 2008. 295(5): p. C1113-22.

5. Ayala, l., et al., Multiple regulatory inputs converge on cortactin to control invadopodia biogenesis and extracellular matrix degradation. J Cell Sci, 2008.

6. Tehrani, S., et al., Src phosphorylation of cortactin enhances actin assembly. Proc Natl Acad Sci U S A, 2007. 104(29): p. 11933-8.

7. Oser, M., et al., Cortactin regulates cofilin and $N$-WASp activities to control the stages of invadopodium assembly and maturation. J Cell Biol, 2009. 186(4): p. 571-87.

8. Oser, M., et al., Specific tyrosine phosphorylation sites on cortactin regulate Nck1-dependent actin polymerization in invadopodia. J Cell Sci, 2010. 123(Pt 21): p. 3662-73.

9. Tomasevic, N., et al., Differential regulation of WASP and N-WASP by Cdc42, Rac1, Nck, and PI(4,5)P2. Biochemistry, 2007. 46(11): p. 3494-502.

10. Padrick, S.B. and M.K. Rosen, Physical mechanisms of signal integration by WASP family proteins. Annu Rev Biochem, 2010. 79: p. 707-35.

11. Martinez-Quiles, N., et al., Erk/Src phosphorylation of cortactin acts as a switch on-switch off mechanism that controls its ability to activate N-WASP. Mol Cell Biol, 2004. 24(12): p. 5269-80.

12. $\mathrm{Wu}, \mathrm{H}$., et al., Identification and characterization of a novel cytoskeletonassociated pp60src substrate. Mol Cell Biol, 1991. 11(10): p. 5113-24.

13. $\mathrm{Wu}, \mathrm{H}$. and J.T. Parsons, Cortactin, an 80/85-kilodalton pp60src substrate, is a filamentous actin-binding protein enriched in the cell cortex. J Cell Biol, 1993. 120(6): p. 1417-26.

14. Huang, C., et al., Down-regulation of the filamentous actin cross-linking activity of cortactin by Src-mediated tyrosine phosphorylation. J Biol Chem, 1997. 272(21): p. 13911-5.

15. van Damme, H., et al., The redistribution of cortactin into cell-matrix contact sites in human carcinoma cells with 11q13 amplification is associated with both overexpression and post-translational modification. J Biol Chem, 1997. 272(11): p. $7374-80$.

16. Lua, B.L. and B.C. Low, Cortactin phosphorylation as a switch for actin cytoskeletal network and cell dynamics control. FEBS Lett, 2005. 579(3): p. 57785. 
17. Cowieson, N.P., et al., Cortactin adopts a globular conformation and bundles actin into sheets. J Biol Chem, 2008. 283(23): p. 16187-93.

18. Zhang, L.H., et al., Dominant expression of $85-\mathrm{kDa}$ form of cortactin in colorectal cancer. J Cancer Res Clin Oncol, 2006. 132(2): p. 113-20.

19. Schiene, C. and G. Fischer, Enzymes that catalyse the restructuring of proteins. Curr Opin Struct Biol, 2000. 10(1): p. 40-5.

20. Weaver, A.M., et al., Interaction of cortactin and N-WASp with Arp2/3 complex. Curr Biol, 2002. 12(15): p. 1270-8. 
A

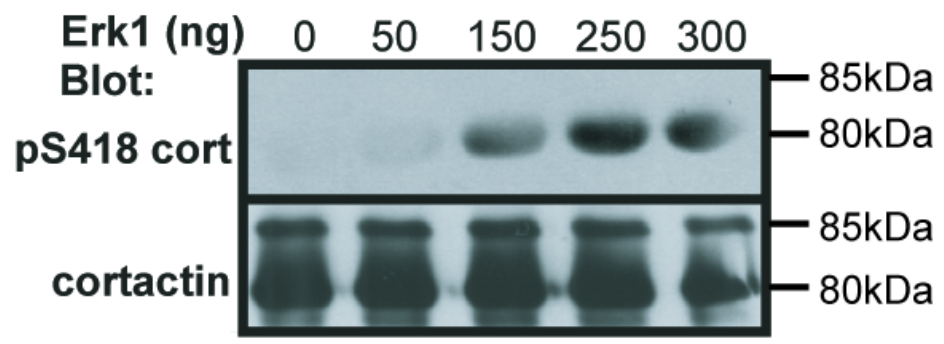

B

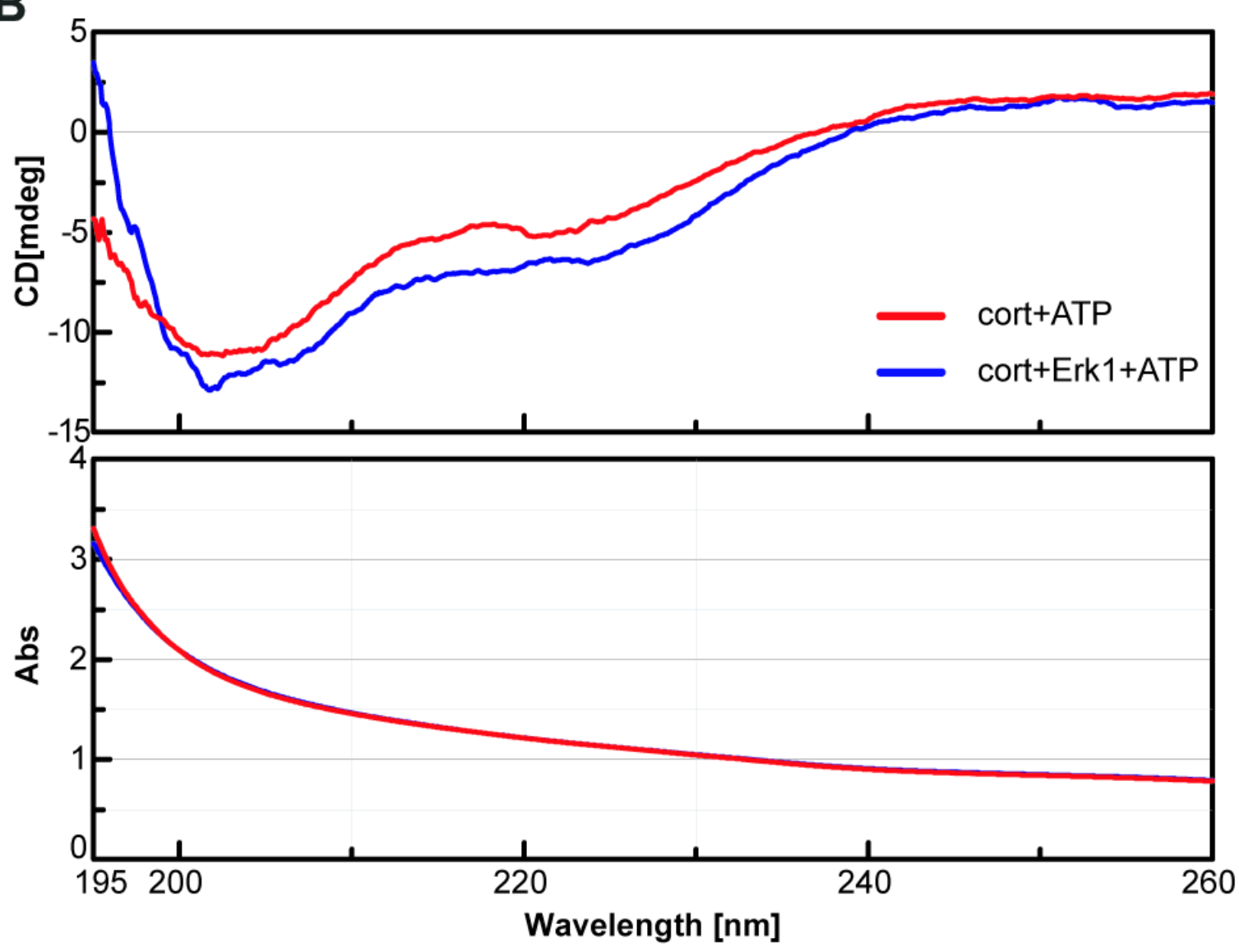

Evans et al. Figure 1 


\title{
Study 3: Construction of a FRET-based Cortactin Biosensor
}

\begin{abstract}
The discovery of fluorescent resonance energy transfer (FRET) in 1946 by Theodore Förster is an invaluable tool for cell biologists, allowing for the spatiotemporal study of individual proteins within specific cellular compartments. One tool that has emerged from this discovery are FRET-based biosensors that give information on a wide array of protein functions. Here we have created a dual tag YFP/CFP biosensor for the actin cytoskeletal protein cortactin. Our preliminary data shows that cortactin activity can be measured and followed by looking at the presence/absence of FRET signal throughout the cellular cytoplasm. This work promises to give rise to an important functional tool that will advance our understanding of cortactin function in regulating cortical actin cytoskeletal networks.
\end{abstract}

\section{Introduction}

Biosensors have become an invaluable tool in recent years for studying the spatiotemporal dynamics of protein activity in fixed and living cells. Biosensor usage has increased in the past two decades with technical advances in light microscopy. These tools enhance the ability to study individual proteins on subcellular levels. This is achieved by providing a readout of the inherent changes in activity and conformation within the protein. One key feature of biosensors is the usage of a biophysical phenomenon known as fluorescent resonance energy transfer, or FRET. 
The beginnings of the concept of FRET were discovered by the father-son team of Jean-Baptiste and Francis Perrin in the 1920s. Together they discovered energy transfer between two identical molecules that were connected by intermolecular dipoledipole interactions and were the first to recognize that this transfer of energy is distancedependent. However, their initial calculation of the efficient distance for energy transfer (15-25nm) was too high. It wasn't until 1946 that Theodore Förster worked out the correct equation for determining the correct distance in which FRET can occur, which is 10nm [1]. Through his equation, he also determined that FRET is dependent upon two other factors in addition to distance. The first is the orientation of the energy donating and energy accepting molecules in relation to one another. Maximum efficiency occurs when the molecule are aligned parallel to with the other. The second factor is that the two molecules must have an emission spectra (donor) and excitation spectra (acceptor) that overlap one another [2]. These unique features allow scientists to apply this technique to design FRET-based biosensor to visually evaluate conformational changes and molecular interactions associated with any aspect of intracellular signaling. Applications of FRET biosensors include using molecules as sensors for determining ligand concentrations $[3,4]$, reporting protein activation states [5-7], translocation of second messengers $[8,9]$, protein conformational changes [10], activation of untagged proteins [11, 12], and detection of protein post-translational modifications [13]. Further applications are likely to be discovered in the future [14].

A protein that may be well suited for biosensor design is the cortical actin-binding protein cortactin. As previously noted, phosphorylation of cortactin by Erk and Src has been implied to drive changes in cortactin conformation, supported by prediction of a 
notion that cortactin must undergo some degree of conformational reordering to allow binding to the phosphorylated tyrosines or to free the $\mathrm{SH} 3$ domain.

In this preliminary study, we have designed a cortactin biosensor that can provide a readout of altered cortactin conformation, providing a means to directly measure cortactin "activity". The biosensor is designed with a yellow fluorescent protein (YFP) tag on the N-terminus of cortactin and a cyan fluorescent protein (CFP) tag on the Cterminal end. This type of FRET biosensor is beneficial in that the ratio of donor and acceptor fluorophores remain equal [21]. We propose that in its closed, or "inactive", conformation, a FRET signal due will be detected due to the close proximity of the YFPCFP FRET pair. Cortactin phosphorylation or $\mathrm{SH} 3$ domain binding is predicted to open the cortactin molecule, disrupting and thus altering the YFP-CFP FRET signal. Our preliminary data with various control constructs would indicate that this proposed model holds true. The cortactin biosensor construct should therefore prove useful in monitoring cortactin function, providing new insight and spatiotemporal detail of cortactin regulation in areas undergoing cortactin-mediated Arp2/3 actin polymerization.

\section{Results}

We have created a dual tag (YFP/CFP) FRET based biosensor to study the spatiotemporal dynamics of cortactin activity and regulation. We propose a model in which the cortactin biosensor gives a FRET signal when cortactin is in its closed conformation. Serine and/or tyrosine phosphorylation of cortactin then confers a structural change which allows cortactin to interact with its binding partners (Figure 1A). We suspect this phosphorylation event to induce these changes due to the large 
numbers of studies indicating the importance of serine/tyrosine phosphorylation in governing cortactin activity [17]. To perform FRET analysis of this biosensor, proper controls were constructed. Along with the biosensor, we cloned an N-terminally YFP tagged cortactin construct as well as a C-terminally CFP tagged construct (Figure 1B). These allow us to perform the proper analysis for FRET signal changes, mainly by allowing determination and removal of nonspecific YFP and CFP signal. We next used these constructs to perform FRET analysis on fixed, epidermal growth factor-stimulated MTLn3 rat breast adenocarcinoma cell line. This cell line was used because of its high expression of the EFG receptor and that these cells form well defined lamellipodia containing cortactin and Arp2/3 complex. Our preliminary data suggest that our cortactin biosensor functions as predicted, with a strong FRET signal observed at the perinuclear region of the cell (inactive cortactin) and gradual decrease in FRET signal towards the edge of the dominant lamellipodia. This would indicate that as cortactin is recruited to sites of actin polymerization from perinuclear stores, it becomes activated by undergoing a conformational change that results in the observed loss of FRET signal. It should be noted that we see the individual YFP and CFP signals at the lamellipodial edge of the cell, showing that the biosensor has localized to these sites and is functioning properly (Figure $1 \mathrm{C}$ ).

Based on these observations, a second construct was designed to aid in determining if the loss of cortical FRET signal parallels cortactin activity. Expression of these constructs were evaluated (Figure 2A) and schematic summaries demonstrating proposed function are shown (Figure 2B-D). Briefly, the $Y C$ construct is a fusion of the fluorescent tags YFP and CFP separated by a small amino acid linker (a generous gift 
from Dr. Alexander Sorkin, University of Pittsburgh). This molecule will give a FRET signal throughout the cellular cytoplasm with no specific localization to any subcellular compartment. In this case the entire cell should demonstrate a FRET signal, acting as a positive control for FRET visualization (Figure 2B). A second control was designed with cortactin containing tandem YFP/CFP tags located at the C-terminal end of the protein. This arrangement will give a constitutive FRET signal wherever cortactin is localized within the cell (Figure 2C). How these constructs are predicted to function is demonstrated in Figure 2D.

\section{Discussion}

Biosensors allow for the study complex signaling pathways in very fine detail. The ability to study single molecule functionality in subcellular compartments has been an astonishing feat and has led to important discoveries, as well as a more in depth knowledge of protein function. Here we have created a biosensor that monitors the activity of the actin cytoskeletal protein cortactin. Our preliminary data indicates that this tool will serve its intended purpose of detecting cortactin conformational changes in areas of active actin polymerization (e.g.; lamellipodia and invadopodia). This biosensor was designed based off of the N-WASP biosensor created by the Condeelis lab [23]. The functional and predicted structural similarities of N-WASP and cortactin, along with and their presence in the same subcellular compartments, supported the use of N-WASp as the cortactin biosensor template. In support of this, recent structural data concerning cortactin suggests that cortactin does not exist as a rod-shaped molecule as reported [15]. Coweison et al. found through crosslinking studies, circular 
dichroism analysis, and small angle X-ray scattering that cortactin exists as a partial globular protein, with the domains C-terminal of the acidic region and first two cortactin repeats forming the globular portion of the molecule [20]. This structure give rise to the hypothesis that cortactin needs to undergo some form of conformational change in order to function properly and interact with binding partners. Furthermore, since phosphorylation of cortactin is such a key moderator of its activity, we propose that these phosphorylation events serve to trigger cortactin conformational change. Data from our lab show that cortactin does not undergo drastic conformational changes following serine or tyrosine phosphorylation ([24] and unpublished data). However, these studies are unable to detect subtle changes that may be associated with alterations in cortactin activity. Another major caveat to this work is that the crystal structure for cortactin has not yet been elucidated, preventing precise determination for the best location of each fluorophore.

When properly validated, the cortactin biosensor will be an invaluable tool in studying the dynamics of actin-based structures including lamellipodia in motile cells and invadopodia formation in cancer cells. Insight into cortactin activity will provide a realtime look at the formation, duration, and disassembly of these dynamic cellular features that could ultimately lead to better understanding of the actin cytoskeletal as well as cellular responses in disease states. 


\section{Methods}

\section{FRET Biosensor Constructs}

YFP and CFP fluorophores were purchased from Clonetech, Inc. Single tagged human cortactin was cloned into the YFP and CFP backbones using the restriction enzymes BamH1 and Sac1 (Roche). The cortactin biosensor was cloned in two steps: 1. Cloning cortactin into the YFP backbone using BamH1 and Xho1 restriction sites. 2. Subcloing the CFP fluorophore through the addition of Xho1 and Sac1 restriction sites. Biosensor $\mathrm{C}$ was a trimolecular ligation of cortactin containing BamH1 and Xho1 restriction sites and the YFP fluorophore containing Xho1 and Sac1 restriction sites cloned into the BamH1 and Sac1 digest CFP backbone.

\section{Western Blotting}

Constructs were transfected into 293T HEK cell lines. The cells were lysed in RIPA buffer and subjected to SDS-PAGE separation. Proteins were then transferred to nitrocellulose and blotted for the fluorescent tags using the JL-8 anti-fluorescent tag antibody (BD Living Colors).

\section{FRET microscopy}

Microscopy experiments were performed using a Nikon TE-2000 Eclipse and analysis was performed by MetaMorph FRET software. Briefly, images from YFP alone, CFP alone, and Raw FRET were obtained and intensity values were determined for individual cells. These values were entered into the MetaMorph software, which performed background subtractions and calculations to produce a FRET sensitized emission result and image. The image is represented as a gradient "heat map" where black/blue areas represent no/weak FRET signal to red areas of "peak" FRET signal. 


\section{Figure Legends}

Figure 1. Design and testing of a FRET-based cortactin Biosensor. (A) Schematic of cortactin FRET biosensor and proposed mechanism of function. (B) Western blot of control and biosensor constructs. (C) Microscopy FRET analysis of cortactin FRET biosensor in MTLn3 cells.

Figure 2. Creation and schematic of function of cortactin biosensor and controls.

(A) Western blot of control constructs and the cortactin biosensor. (B-D) Schematic of proposed function of $\mathrm{YC}$, Biosensor $\mathrm{C}$, and Biosensor A (cortactin biosensor) constructs during FRET microscopy analysis. 


\section{$\underline{\text { References }}$}

1. Sun, Y., et al., FRET microscopy in 2010: the legacy of Theodor Forster on the 100th anniversary of his birth. Chemphyschem, 2011. 12(3): p. 462-74.

2. Day, R.N. and M.W. Davidson, Fluorescent proteins for FRET microscopy: monitoring protein interactions in living cells. Bioessays, 2012. 34(5): p. 341-50.

3. Miyawaki, A., et al., Fluorescent indicators for $\mathrm{Ca2}+$ based on green fluorescent proteins and calmodulin. Nature, 1997. 388(6645): p. 882-7.

4. Zaccolo, M., et al., A genetically encoded, fluorescent indicator for cyclic AMP in living cells. Nat Cell Biol, 2000. 2(1): p. 25-9.

5. Kraynov, V.S., et al., Localized Rac activation dynamics visualized in living cells. Science, 2000. 290(5490): p. 333-7.

6. Del Pozo, M.A., et al., Integrins regulate GTP-Rac localized effector interactions through dissociation of Rho-GDI. Nat Cell Biol, 2002. 4(3): p. 232-9.

7. Gardiner, E.M., et al., Spatial and temporal analysis of Rac activation during live neutrophil chemotaxis. Curr Biol, 2002. 12(23): p. 2029-34.

8. Hurley, J.H. and T. Meyer, Subcellular targeting by membrane lipids. Curr Opin Cell Biol, 2001. 13(2): p. 146-52.

9. Balla, T., T. Bondeva, and P. Varnai, How accurately can we image inositol lipids in living cells? Trends Pharmacol Sci, 2000. 21(7): p. 238-41.

10. Toutchkine, A., et al., Experimental and DFT studies: novel structural modifications greatly enhance the solvent sensitivity of live cell imaging dyes. J Phys Chem A, 2007. 111(42): p. 1084960.

11. Kalab, P., K. Weis, and R. Heald, Visualization of a Ran-GTP gradient in interphase and mitotic Xenopus egg extracts. Science, 2002. 295(5564): p. 2452-6.

12. Graham, D.L., P.N. Lowe, and P.A. Chalk, A method to measure the interaction of Rac/Cdc42 with their binding partners using fluorescence resonance energy transfer between mutants of green fluorescent protein. Anal Biochem, 2001. 296(2): p. 208-17.

13. Ting, A.Y., et al., Genetically encoded fluorescent reporters of protein tyrosine kinase activities in living cells. Proc Natl Acad Sci U S A, 2001. 98(26): p. 15003-8.

14. Gaits, F. and K. Hahn, Shedding light on cell signaling: interpretation of FRET biosensors. Sci STKE, 2003. 2003(165): p. PE3.

15. Weaver, A.M., et al., Interaction of cortactin and N-WASp with Arp2/3 complex. Curr Biol, 2002. 12(15): p. 1270-8.

16. Weed, S.A., et al., Cortactin localization to sites of actin assembly in lamellipodia requires interactions with F-actin and the Arp2/3 complex. J Cell Biol, 2000. 151(1): p. 29-40.

17. Ammer, A.G. and S.A. Weed, Cortactin branches out: roles in regulating protrusive actin dynamics. Cell Motil Cytoskeleton, 2008. 65(9): p. 687-707.

18. Huang, C., et al., The role of tyrosine phosphorylation of cortactin in the locomotion of endothelial cells. J Biol Chem, 1998. 273(40): p. 25770-6.

19. Campbell, D.H., R.L. Sutherland, and R.J. Daly, Signaling pathways and structural domains required for phosphorylation of EMS1/cortactin. Cancer Res, 1999. 59(20): p. 5376-85.

20. Cowieson, N.P., et al., Cortactin adopts a globular conformation and bundles actin into sheets. J Biol Chem, 2008. 283(23): p. 16187-93.

21. Herbst, K.J., Q. Ni, and J. Zhang, Dynamic visualization of signal transduction in living cells: from second messengers to kinases. IUBMB Life, 2009. 61(9): p. 902-8.

22. Weed, S.A., Y. Du, and J.T. Parsons, Translocation of cortactin to the cell periphery is mediated by the small GTPase Rac1. J Cell Sci, 1998. 111 ( Pt 16): p. 2433-43. 
23. Lorenz, M., et al., Imaging sites of $\mathrm{N}$-wasp activity in lamellipodia and invadopodia of carcinoma cells. Curr Biol, 2004. 14(8): p. 697-703.

24. Evans, J.V., et al., Further insights into cortactin conformational regulation. Bioarchitecture, 2011. 1(1): p. 21-23. 
A.

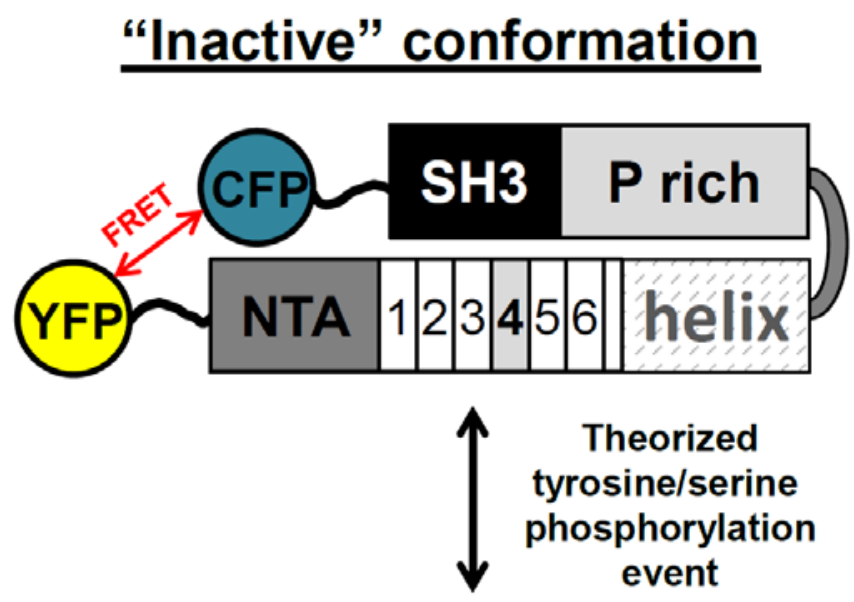

"Active" conformation

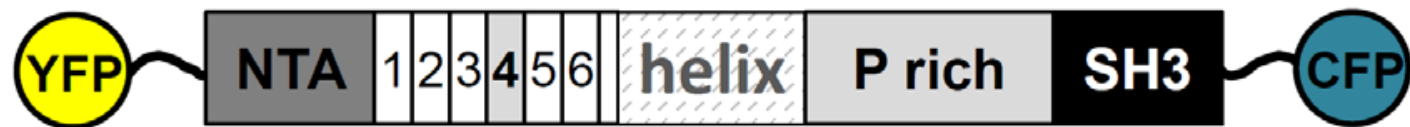

B.

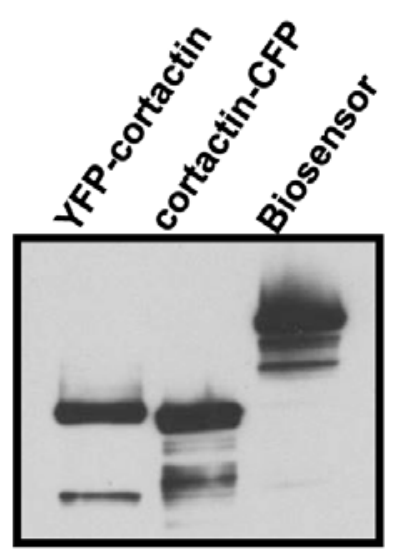

C.

Cortactin Biosensor
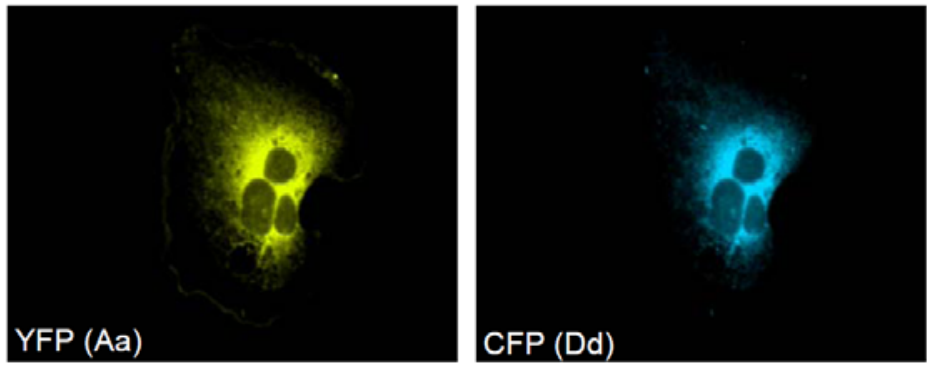

Raw FRET (Da)

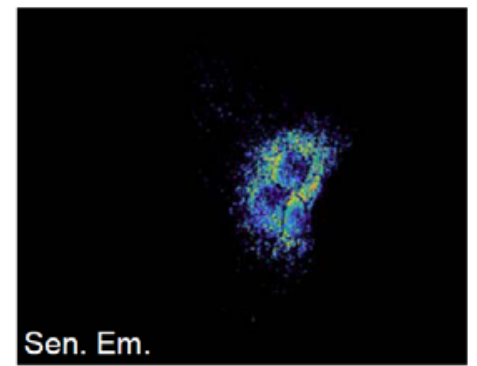

Figure 1. 
A.

B.

YC: Global FRET signal
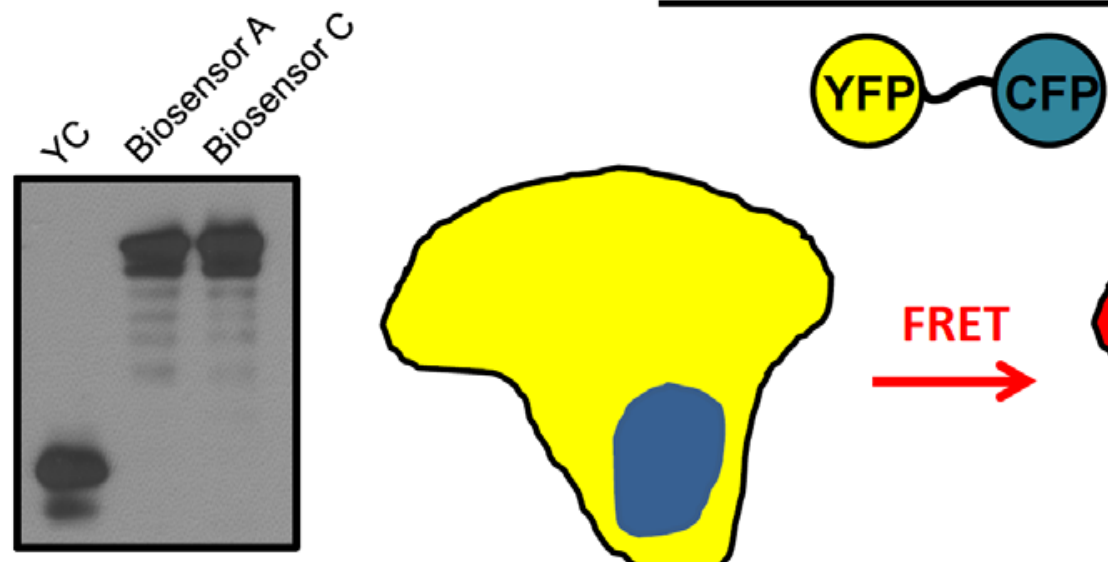

Yellow=YFP signal

Red=FRET signal
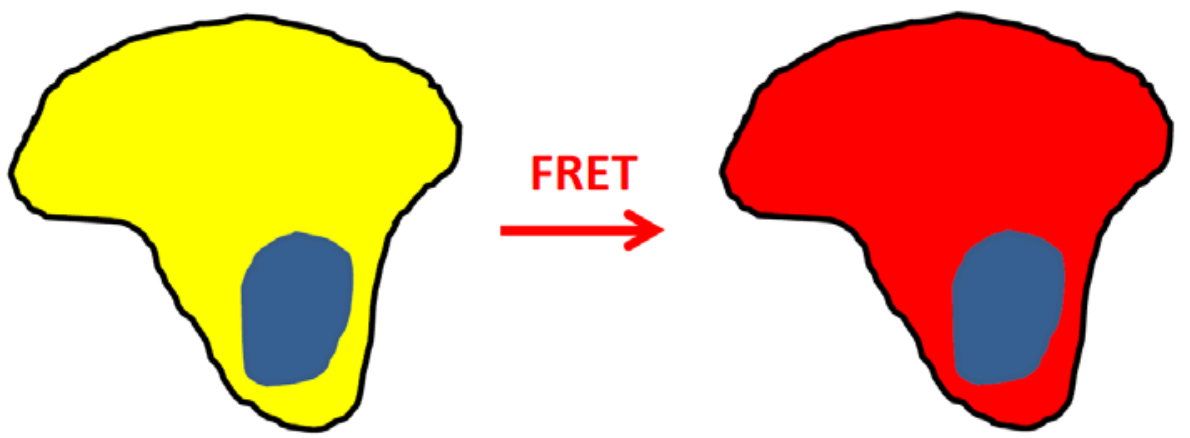

C.

\section{Biosensor C: Cortactin localization FRET signal}
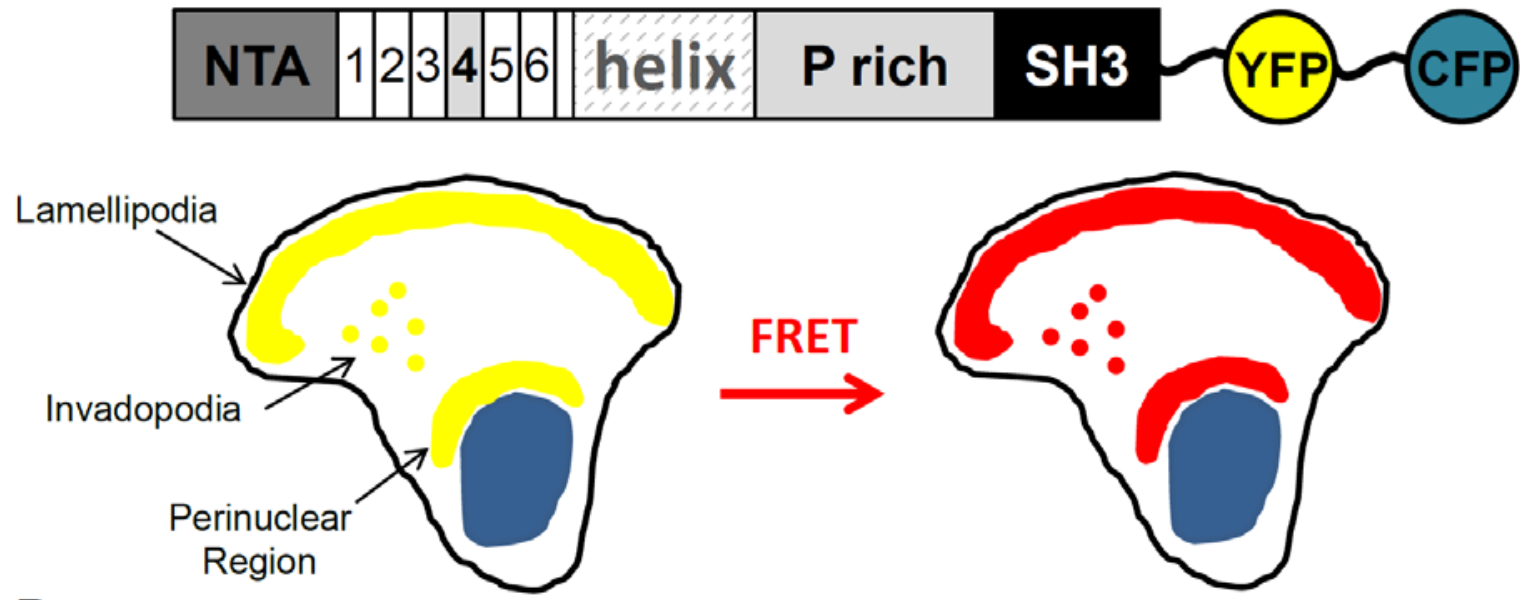

D.

Biosensor A: Cortactin activity FRET signal loss
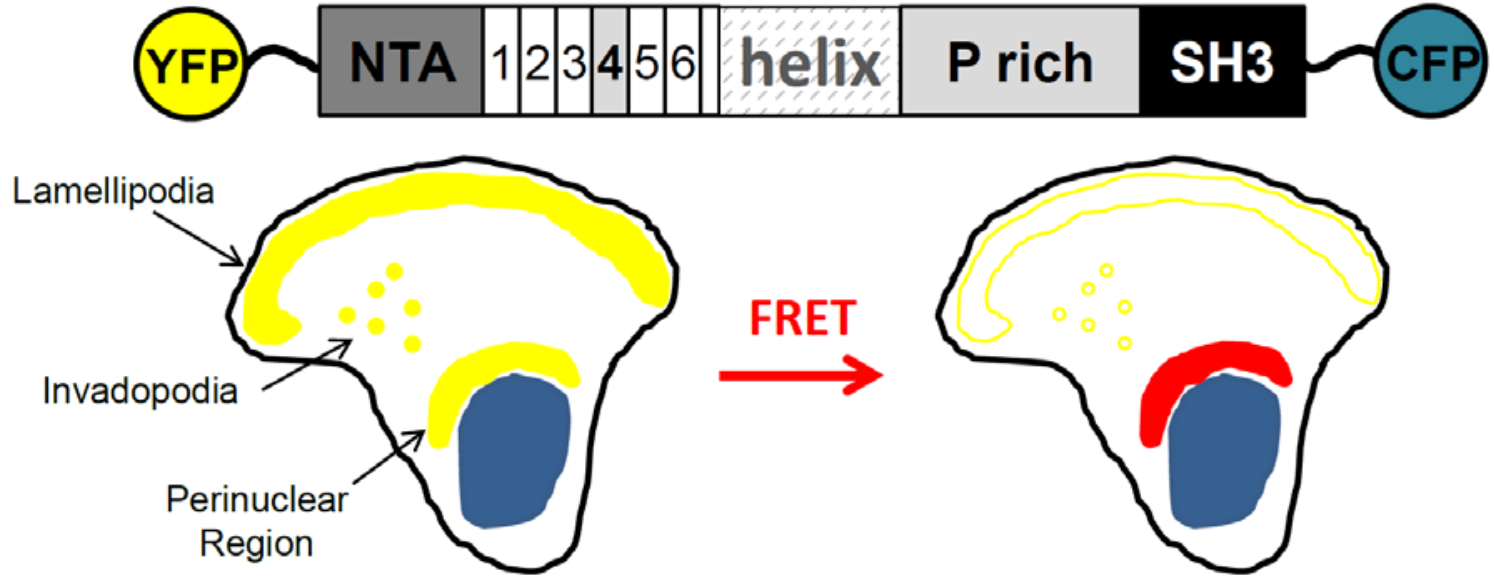

Figure 2. 


\section{$\underline{\text { Discussion }}$}

Src and cortactin have an interesting, intermingled history. From the initial discovery of cortactin as a hyperphosphorylated $80 / 85 \mathrm{kD}$ protein in Src transformed fibroblasts to recent work on Src and cortactin in invadopodia regulation [1, 2], it has become abundantly clear that Src is a key regulator of cortactin function through phosphorylation of Y421, Y466, and Y482 [3]. Cortactin function is central to proper formation of branched actin networks and the dynamics in which these networks are formed and dissolved. It performs this role through two main mechanisms. One is to act as a scaffolding protein for proteins essential in controlling actin dynamics. Cortactin is able to interact directly with F-actin filaments. With this trait, its interactions with binding partners bring these proteins in close proximity to these locations of active filament growth where they can perform their respective regulatory functions [4]. The other mechanism of cortactin action is to act as an NPF. Here cortactin is responsible for regulating activation of the Arp2/3 complex. This critical creation of a branched network of actin filaments is what gives cells their shape and promotes processes such as cell motility, protrusion, and adhesion.[5].

Essentially, cortactin acts as a key intersection point for multiple signaling pathways that impinge on actin cytoskeletal regulation. To date regulation of cortactin by Src is mostly measured by the levels of cortactin tyrosine phosphorylation. This was primarily due to the incomplete understanding of the binding mechanism between Src and, as it was assumed to be mediated by the Src SH2 domain binding to cortactin pY421 and/or pY466 [6]. Our work in Study 1 details this missing mechanism as well as the previously unrecognized signaling pathways that potentially control this interaction. 
In the past decade there have been conflicting data debating the differential roles of serine and tyrosine phosphorylation of cortactin has on actin filament assembly $[7,8]$. From this, it has emerged that Src is the primary kinase that is responsible for cortactin tyrosine phosphorylation and this promotes actin polymerization. Our work from Study 1 supports these claims as well [9]. However, these studies do not address the idea of cortactin undergoing a conformational change upon serine and/or tyrosine phosphorylation. This concept gained traction with data from Coweison et al. that showed, through circular dichroism analysis, chemical crosslinking, and small angle $\mathrm{x}$ ray scattering, that cortactin holds to a "lollipop" conformation. In this model, the acidic domain and a portion of the repeat region (how many repeats is not known) forms the "stick" of the lollipop, while the rest of the C-terminal portion folds into a globular state [10]. This gives rise to the question of how the tyrosine/serine residues and the $\mathrm{SH} 3$ domain is situated to allow cortactin to function properly. This also gives credence to the idea of conformational regulation of cortactin function. In Study 2, we sought to answer this question using Erk [9] and Src (data not shown) kinase assays and circular dirchroism. This work was performed on the heels the study from our lab by Kelley et al., where it was shown that upon EGF stimulation the cortactin doublet band shifts to just one band, possibly indicating a conformational shift [11]. This data confirms previous studies $[12,13]$. Our data indicates that if a conformational change exists at all, then it is very small. CD spectra from serine phosphorylated cortactin only showed minute shift in spectra, but the overall shape of the curve did not indicate we were seeing global changes in molecular confirmation. Similar results were seen with Src tyrosine phosphorylated cortactin. One caveat is that CD spectra are difficult to use as 
a stand-alone readout. Generally, this data is coupled with NMR, x-ray scattering, or crystallography data and, unfortunately, a large hole in the cortactin field is that the crystal structure of cortactin has never been solved. In fact, up until the "lollipop" study, cortactin was thought to hold a "rod shaped" conformation, in which it is fully laid out from end to end, with little or no tertiary structure [5]. Once the crystal structure of cortactin is determined, it will be exciting to see what interesting studies emerge; aimed at discovering the exact molecular mechanisms of cortactin function and how it interacts with dynamic actin complexes.

In a more elegant attempt to study cortactin conformational changes detailed in Study 3, we created a FRET-based cortactin biosensor. This tool is designed to give a sensitive readout of minute changes in cortactin conformation that will lead to altered levels of FRET activity. Biosensors have been widely used in the study of protein function within the cell and, when properly controlled, have given beautifully informative spatial-temporal images and analyses of protein function and activity. Although very preliminary, our data indicates that the cortactin biosensor undergoes enough of a conformational shift that we see differential FRET signals within areas of cortactin localization. We predict cortactin to give a FRET signal when in its "closed" or inactive conformation. Upon localization to sites of actin cytoskeletal formation, we see a loss in this FRET signal which would indicate a change in conformation or "open" conformation, allowing cortactin to actively participate in cytoskeletal signaling events. This data looks at cortactin in the context of lamellipodia formation at the leading edge of the cell. As we know, cortactin is located in the perinuclear region of the cytoplasm and upon Rac1 activation is translocated to the cell edge [14]. This activation process falls in line with 
our data, in that we see a FRET signal at the perinuclear region (inactive) and then observe a gradient loss of FRET signal as we move out toward the leading edge of the cell (active), indicating that the biosensor may be a useful tool for studying cortactin function in multiple cellular settings such as endocytosis and invadopodia formation. Again, the biggest caveat is because of a lack of cortactin crystal structure, we cannot fully determine if our biosensor is truly an indicator of cortactin conformational changes as it regards to activity. However, we do feel that with the proper controls, as the ones we have created, we can, with relative confidence, apply this biosensor in a meaningful way.

Much work has been performed looking at the cellular and downstream signaling events that result from the Src-mediated tyrosine phosphorylation of cortactin. Until our work, the exact mechanism of binding of cortactin was not known other than it was mediated by the Src SH2 domain. Our work has ultimately shed light upon this mystery and has opened a mechanistically novel mode of interaction for $\mathrm{SH} 2$ domains that are subject to regulation in a previously unrealized manner. We have shown that a cysteine residue in the hydrophobic pocket of the Src SH2 domain (C185) forms a disulfide bond with two separate cysteine residues within the cortactin repeats. C185 of Src has been previously noted in the literature. In a study looking at the mechanisms of specificity of the Src SH2 domain, it was shown that when this cysteine was mutated to alanine that an 8-fold increase in affinity for phosphotyrosine peptides was observed. This gave rise to the idea that the negative charge located on the deprotonated cysteine residue provides a repulsive negative charge to the oxygen moieties on the phosphate group of the tyrosines. This would allow an easier detachment from these residues, allowing 
dynamic binding and unbinding events to occur [15]. This means of binding between Src and cortactin is unique, to say the least. It is generally thought that kinase interactions with their substrates are transient in nature. However, we find the formation of a stable bond that is necessary for cellular functions. Our data shows that when this bond is disrupted, HNSCC cells are unable to form their spontaneous, invasive structures, known as invadopodia. It's not the phosphorylation that is necessary, as has been shown to be the case for many cellular processes, but the binding event itself. This would suggest that this stable interaction is necessary to act as the core foundation upon which all other components of invadopodia build upon.

This unique interaction also brings up the question of "how it is regulated?" The short answer is that it has not been determined as of yet. However, there are instances in the literature that shed light on possible mechanisms. The cytoplasm is largely a reducing environment due to the ample presence of glutathione. This type of environment would not be conducive to the formation of stable disulfide bonds. However, recent reports by two laboratories indicate that focal sites of oxidative environments can persist. This work has shown that invadopodia formation in colon cancer cells is dependent upon Tks5 generated ROS, which creates an oxidative pocket within the cell. Without this ROS production, the formation of invadopodia is inhibited $[16,17]$. Other data that supports this is that upon hydrogen peroxide treatment of cells, there is an increase in Src-dependent tyrosine phosphorylation of cortactin, indicating that an increase in hydrogen peroxide induced ROS promotes the Src/cortactin interaction [18]. There are also other instances of disulfide bonds being able to form in the cellular cytoplasm, however, how these form has not been studied extensively [19- 
21]. So far, this explains how this bond can form in the cytoplasm, but neglects how such a stable bond can be broken. The simplest answer is that upon dissolution of the invadopodia structure, the oxidative environment is quickly dissipated and reduced by the large presence of cytoplasmic glutathione. Another alternative is that proteins known as oxidoreductases may perform this function. Particularly, the PDI family of thiol oxidoreductases has been shown to localize to the cell surface, which is a unique feature since most oxidoreductases are found in the endoplasmic reticulum where they play a critical role in the proper folding of newly translated proteins [22]. This new mechanism of $\mathrm{SH} 2$ domain mediated interaction gives credence to analyzing the levels and localization of ROS in cells and promoting future studies to address this as a means of regulation of intracellular processes. Interestingly, sequence alignment of all known $\mathrm{SH} 2$ domains indicate that this mode of interaction may be more widespread than the $\mathrm{Src} \mathrm{SH} 2$ domain, possibly encompassing $25 \%$ of these domains over a wide array of protein classes.

In summation, the major focus of this work details a novel mode of interaction for the $\mathrm{Src} \mathrm{SH} 2$ domain. Non-conventional, phospho-tyrosine independent interactions between $\mathrm{SH} 2$ domains and their substrates have been documented; however, these have been shown to be weaker, electrostatic interactions. Our work identifying a cystine bond formation between Src and cortactin opens a new paradigm to how proteins interact with one another. Turning our focus to understanding protein-protein interactions may prove to be beneficial in targeting certain molecular pathways in order to treat disease states such as cancer. Much emphasis has been put on inhibiting the functionality of proteins in cancer cells, mainly through inhibiting kinetic activity of 
particular enzymes, such as Src. Preventing specific protein interactions may be enough to help trigger a response in these disease states while eliminating unintended side effects. As we have shown, the simple elimination of the ability of two proteins to interact has had detrimental effects on the ability of HNSCC cells to form invasive, metastatic structures. We have provided a potential mechanism of attack to prevent HNSCC from turning into metastatic disease, which could enhance patient survival. Additionally, this work exemplifies that ligand-receptor based signaling pathways may not be the sole driving force in cancer progression, as the current research trend seems to support. In closing, we feel that we have illustrated a significant, paradigm shifting mechanism that has implications in metastatic tumor cell signaling. This work also opens the door to new ideas about how proteins can interact with one another and how the signaling events that control these interactions may be infinitely more complicated and intricate than previously thought. 


\section{$\underline{\text { References }}$}

1. $\mathrm{Wu}, \mathrm{H}$. , et al., Identification and characterization of a novel cytoskeleton-associated pp60src substrate. Mol Cell Biol, 1991. 11(10): p. 5113-24.

2. Hayes, K.E., et al., Ableson kinases negatively regulate invadopodia function and invasion in head and neck squamous cell carcinoma by inhibiting an HB-EGF autocrine loop. Oncogene, 2012.

3. Ammer, A.G. and S.A. Weed, Cortactin branches out: roles in regulating protrusive actin dynamics. Cell Motil Cytoskeleton, 2008. 65(9): p. 687-707.

4. Weed, S.A., et al., Cortactin localization to sites of actin assembly in lamellipodia requires interactions with F-actin and the Arp2/3 complex. J Cell Biol, 2000. 151(1): p. 29-40.

5. Weaver, A.M., et al., Interaction of cortactin and N-WASp with Arp2/3 complex. Curr Biol, 2002. 12(15): p. 1270-8.

6. Weed, S.A. and J.T. Parsons, Cortactin: coupling membrane dynamics to cortical actin assembly. Oncogene, 2001. 20(44): p. 6418-34.

7. Martinez-Quiles, N., et al., Erk/Src phosphorylation of cortactin acts as a switch on-switch off mechanism that controls its ability to activate N-WASP. Mol Cell Biol, 2004. 24(12): p. 5269-80.

8. Tehrani, S., et al., Src phosphorylation of cortactin enhances actin assembly. Proc Natl Acad Sci U S A, 2007. 104(29): p. 11933-8.

9. Evans, J.V., et al., Further insights into cortactin conformational regulation. Bioarchitecture, 2012. 1(1): p. 21-23.

10. Cowieson, N.P., et al., Cortactin adopts a globular conformation and bundles actin into sheets. J Biol Chem, 2008. 283(23): p. 16187-93.

11. Kelley, L.C., et al., Cortactin phosphorylated by ERK1/2 localizes to sites of dynamic actin regulation and is required for carcinoma lamellipodia persistence. PLoS One, 2010. 5(11): p. e13847.

12. van Damme, $\mathrm{H}$., et al., The redistribution of cortactin into cell-matrix contact sites in human carcinoma cells with 11q13 amplification is associated with both overexpression and posttranslational modification. J Biol Chem, 1997. 272(11): p. 7374-80.

13. Campbell, D.H., R.L. Sutherland, and R.J. Daly, Signaling pathways and structural domains required for phosphorylation of EMS1/cortactin. Cancer Res, 1999. 59(20): p. 5376-85.

14. Weed, S.A., Y. Du, and J.T. Parsons, Translocation of cortactin to the cell periphery is mediated by the small GTPase Rac1. J Cell Sci, 1998. 111 ( Pt 16): p. 2433-43.

15. Charifson, P.S., et al., Peptide ligands of pp60(c-src) SH2 domains: a thermodynamic and structural study. Biochemistry, 1997. 36(21): p. 6283-93.

16. Diaz, B., et al., Tks5-dependent, nox-mediated generation of reactive oxygen species is necessary for invadopodia formation. Sci Signal, 2009. 2(88): p. ra53.

17. Gianni, D., et al., Novel p47(phox)-related organizers regulate localized NADPH oxidase 1 (Nox1) activity. Sci Signal, 2009. 2(88): p. ra54.

18. Li, Y., J. Liu, and X. Zhan, Tyrosine phosphorylation of cortactin is required for H2O2-mediated injury of human endothelial cells. J Biol Chem, 2000. 275(47): p. 37187-93.

19. Cumming, R.C., Analysis of global and specific changes in the disulfide proteome using redox two-dimensional polyacrylamide gel electrophoresis. Methods Mol Biol, 2008. 476: p. 165-79.

20. Cumming, R.C., et al., Protein disulfide bond formation in the cytoplasm during oxidative stress. J Biol Chem, 2004. 279(21): p. 21749-58.

21. Cumming, R.C. and D. Schubert, Amyloid-beta induces disulfide bonding and aggregation of GAPDH in Alzheimer's disease. FASEB J, 2005. 19(14): p. 2060-2.

22. Turano, C., et al., Proteins of the PDI family: unpredicted non-ER locations and functions. J Cell Physiol, 2002. 193(2): p. 154-63. 


\section{Jason V. Evans}

2536 Stewartstown Road, Morgantown, WV 26508

Home: (304) 922-8188 • Work: (304) 922-8188 or (304) 293-3090

E-mail: jevans@hsc.wvu.edu

\section{Education}

- Ph.D., Cancer Cell Biology Program, Department of Neuroscience and Anatomy West Virginia University, Morgantown, WV 2004-Present

- B. S., Medical Technology, Magna Cum Laude, Department of Pathology West Virginia University, Morgantown, WV 2004

\section{Certification}

- American Society for Clinical Pathology (ASCP) : Sept. 2004-Sept. 2007

\section{Professional Development}

- The American Society for Cell Biology $50^{\text {th }}$ Annual Meeting, Philadelphia, PA December 11-15, 2010

- $\quad 3^{\text {rd }}$ Biennial IDeA Symposium, Bethesda, MD June 16-18, 2010

- Imaging with Metamorph Workshop, Downingtown, PA September 11-12, 2006

\section{Graduate Course Work}

- Cell Structure and Metabolism

- Fundamentals of Integrated Systems

- Biostatistics

- Introduction into Biomedical Research (ethics course)

- Molecular Genetics 
- Cell Signaling and Metabolism

- Cancer Cell Biology

- Scientific Writing

- Basic and Clinical Aspects of Cancer

- Oncogenes and Signaling Networks

- Cancer Therapeutics

\section{Technical Abilities}

- Biochemical protein analysis and purification: Western/Far Western blotting, immunoprecipitation, affinity precipitation, recombinant protein production, rat tail tendon collagen purification, sample preparation for tandem mass spectroscopy

- Circular dicroism spectroscopy

- Cellular migration, adhesion, and invasion assays: ECIS, reverse migration assays, single cell tracking, wound healing assays, gelatin degradation, Boyden chamber based assays, Oris cell migration assay

- Fluorescent/Confocal microscopy

- FRET-based microscopy

- DNA cloning/mutagenesis

- Tissue/Cell culture techniques: maintaining cell lines, cellular transfection, lentivirus preparation and subsequent cellular infection

- Biosensor Design

- Kinase assays

\section{Scholarly Activities}

- Cell Motility and Migration Consortium: A bi-weekly meeting of several labs whose research focus is studying the mechanisms of cellular movement/invasion. 
- Cell Biology Training Program: A program to introduce graduate students to a wide variety of cellular/biochemical/microscopy techniques that they may not experience in their respective labs. Founding student member.

- Journal Club: Weekly discussion and analysis of the most current topics in cancer biology/therapeutics.

- Cancer Cell Biology Student Research Meeting: A bi-weekly, informal, student-based meeting focused on discussing and troubleshooting thesis projects, as well as encouraging networking between graduate students of different labs.

- Van Liere Research Day: West Virginia University's yearly celebration of the biomedical research being conducted at its Health Sciences Center campus. Poster presented in 2007 and 2009.

- Student Seminar Presentations: Formal seminars given annually that are designed to allow students to present their research and to equip them with the fundamentals of scientific speaking in front of faculty and peers.

\section{Teaching/Training Experience}

- Training summer INBRE and COBRE students, undergraduate researchers, and rotational Ph.D. students on how to perform various laboratory techniques and explaining background literature pertinent to the cortactin field of study.

\section{Publications}

- Src Binds Cortactin Through a SH2 Domain Cystine-mediated Linkage, Jason V. Evans, Amanda G. Ammer, John E. Jett, Chris A. Bolcato, Jason C. Breaux, Karen H. Martin, Mark V. Culp, Peter M. Gannett, and Scott A. Weed Journal of Cell Science 2012 Oct 2; in press

- Further Insights into Cortactin Conformational Regulation, Jason V. Evans, Laura C. Kelley, Karen E. Hayes, Amanda Gatesman Ammer, Karen H. Martin and Scott A. Weed Bioarchitecture 2011 Jan;1(1):21-23.

- Sarcatinib Impairs Head and Neck Squamous Cell Carcinoma Invasion by Disrupting Invadopodia Function, Ammer AG, Kelley LC, Hayes KE, Evans JV, Lopez-Skinner LA, Martin KH, Frederick B, Rothschild BL, Raben D, Elvin P, Green TP, Weed SA J Cancer Sci Ther. 2009 Nov 30;1(2):52-61 


\section{Research Interests}

- Cancer cell signal transduction and metabolism

- Mechanism of homing of tumor cells to specific metastatic sites

- Molecular biology of cancer progression, metastasis, and interaction with surrounding microenvironment

- Cancer stem cell signaling and therapeutic targeting

- Animal models of human disease

\section{Future Goals}

- To attain an academic post-doctoral position that will allow me to mature, mentally and technically, as an independent scientist. This is in preparation for a career guiding and sustaining a biomedical research laboratory in academia.

- To hone the necessary skills to write clear and effective grants to sustain research funding.

- To work in a vibrant, collaborative, and sociable environment that promotes sharing and learning of scientific ideas. 